Aus der Klinik für Neurochirurgie

( Prof. Dr. med. V. Rohde )

der Medizinischen Fakultät der Universität Göttingen

\title{
Analyse der Schraubenfehllage bei roboterassistierten perkutanen chirurgischen Verfahren der thorakolumbalen Wirbelsäule
}

\author{
INAUGURAL-DISSERTATION \\ zur Erlangung des Doktorgrades \\ der Medizinischen Fakultät \\ der Georg-August-Universität zu Göttingen
}

vorgelegt von

Anina Isabel Hahn

aus

Bonn

Göttingen 2018 
Dekan: $\quad$ Prof. Dr. rer. nat. H. K. Kroemer

Referent: $\quad$ Prof. Dr. med. R. Martínez-Olivera

Ko-Referent: Prof. Dr. med. René Müller-Wille

Drittreferent: Prof. Dr. med. Martin Oppermann

Datum der mündlichen Prüfung: 14.08.2019 
Hiermit erkläre ich, die Dissertation mit dem Titel „Analyse der Schraubenfehllage bei roboterassistierten perkutanen chirurgischen Verfahren der thorakolumbalen Wirbelsäule“ eigenständig angefertigt und keine anderen als die mir angegebenen Quellen und Hilfsmittel verwendet zu haben.

Göttingen, den 30.04.2018

(Anina Isabel Hahn) 


\section{Inhaltsverzeichnis}

Abbildungsverzeichnis III

Tabellenverzeichnis IV

Abkürzungsverzeichnis $\quad$ V

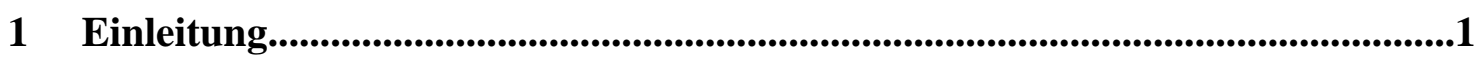

1.1 Zielsetzung und Aufgabenstellung der Arbeit....................................................... 1

1.2 Klinische Bedeutung der Pedikelschraubenfehlplatzierung..................................2

1.3 Entwicklung und Ergebnisse der nicht-navigationsassistierten Spondylodese........2

1.4 Entwicklung und Ergebnisse der navigationsassistierten Spondylodese .................4

1.5 Besondere Patientengruppen bei Pedikelschraubeninsertion.................................

1.6 Perkutane versus offene Operationstechnik und ihr Stellenwert bei navigationsund roboterassistierter Pedikelverschraubung.....................................................

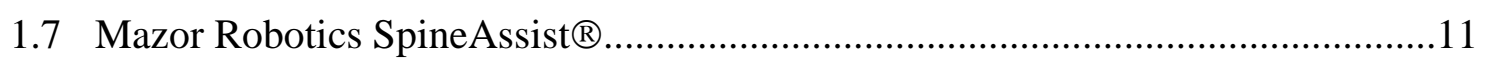

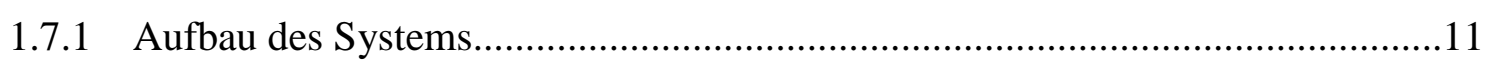

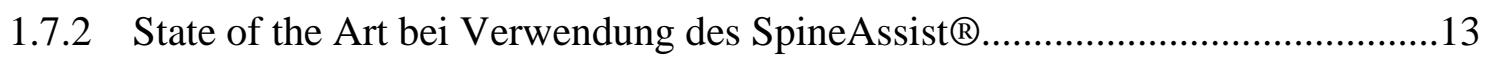

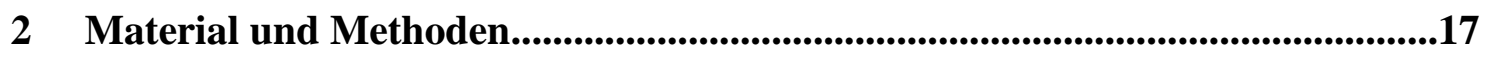

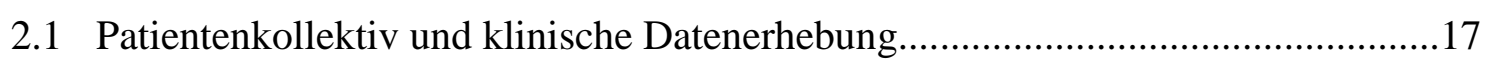

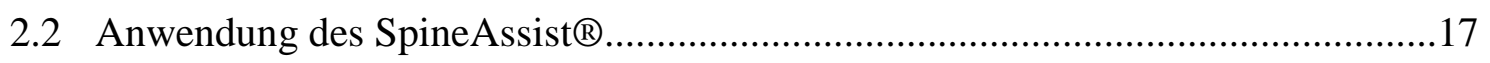

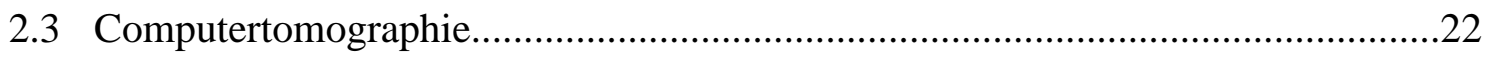

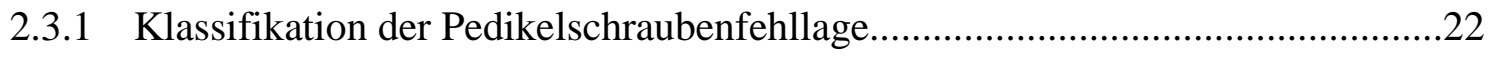

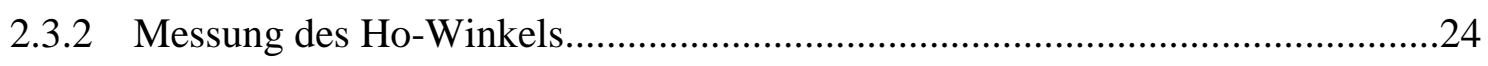

2.3.3 Messung der Knochendichte in Hounsfieldeinheiten.........................................25

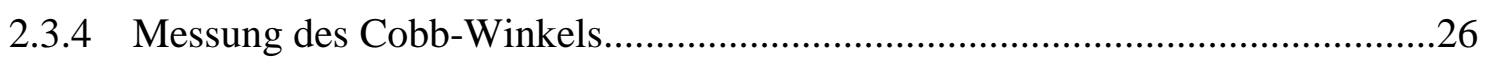

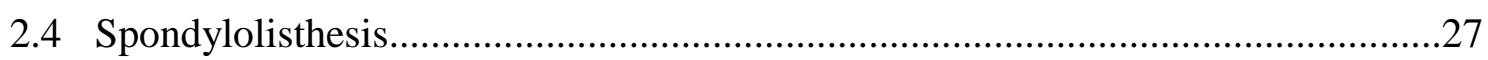

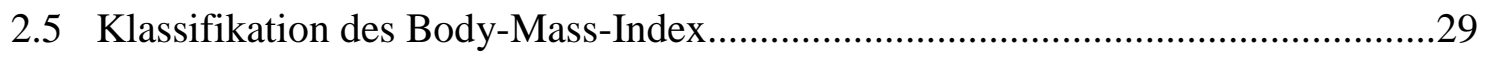

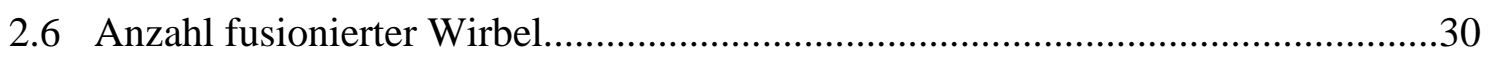

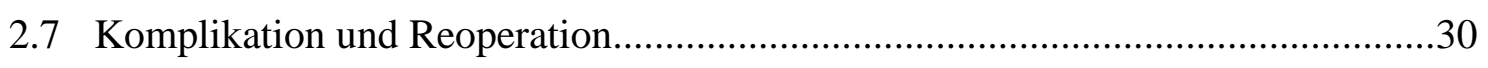

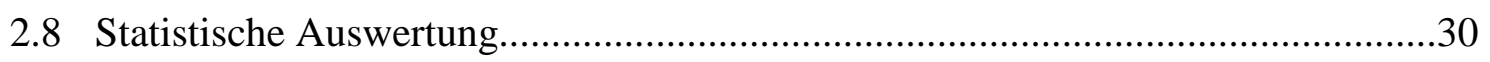

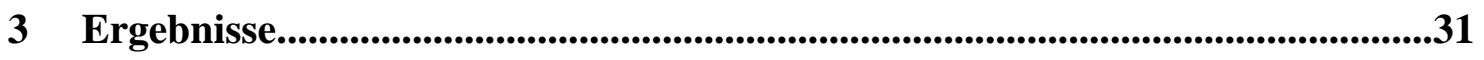

3.1 Verteilung der Pedikelschrauben....................................................................... 31

3.2 Verteilung korrekter und fehlplatzierter Pedikelschrauben....................................32

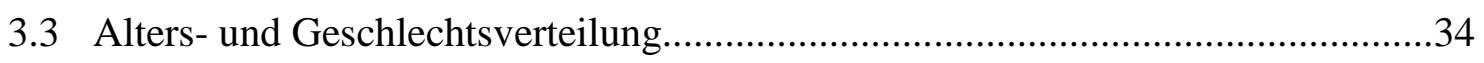




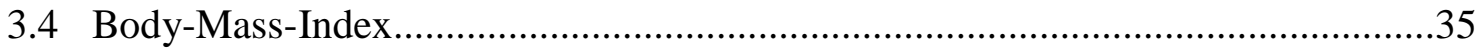

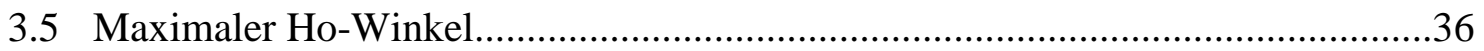

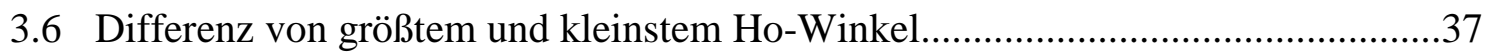

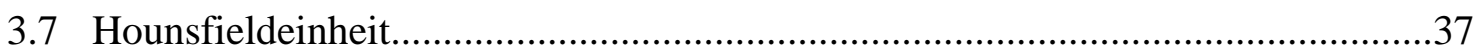

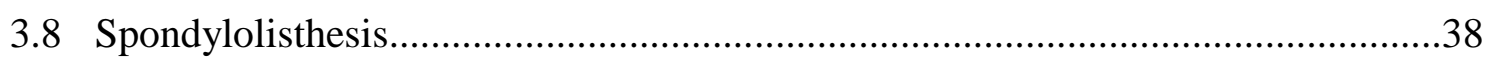

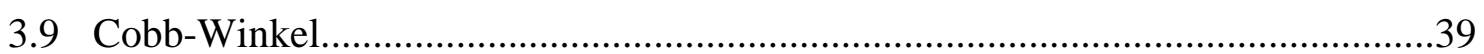

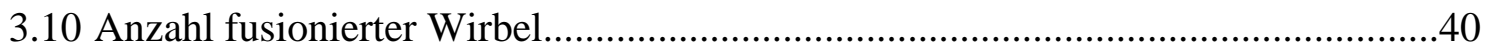

3.11 Tabellarische Zusammenfassung der statisischen Auswertung.............................42

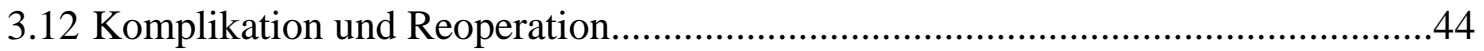

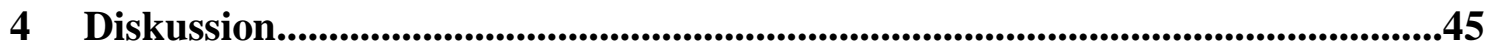

4.1 Genauigkeit der Schraubenpositionierung durch den SpineAssist ${ }^{\circledR}$........................45

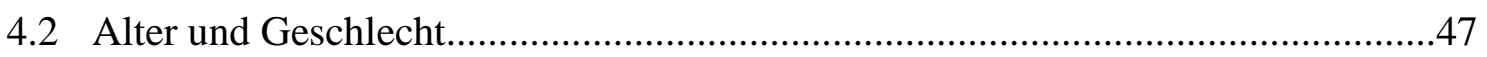

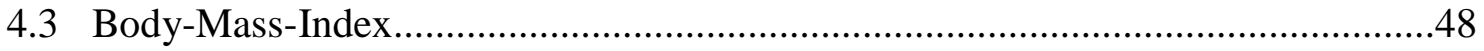

4.4 Maximaler Ho-Winkel und Differenz von größtem und kleinstem Ho-Winkel.....50

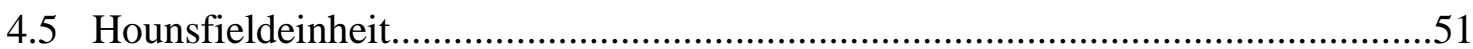

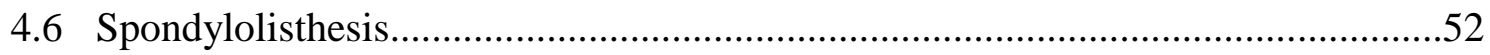

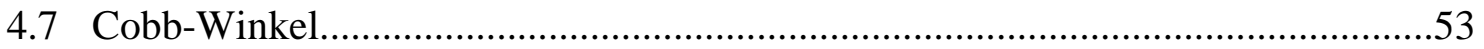

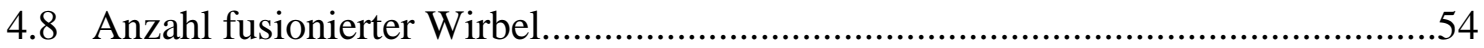

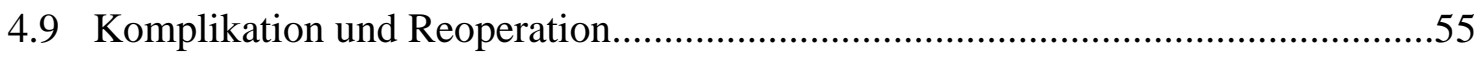

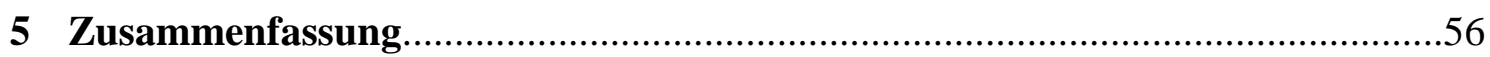

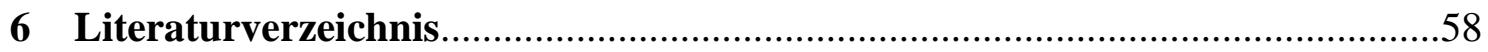




\section{Abbildungsverzeichnis}

Abb. 1: $\quad$ SpineAssist ${ }^{\circledR}$ Roboter mit Zubehör 12

Abb. 2: Präoperative Planung der Verlaufsbahn der Pedikelschrauben .18

Abb. 3: Kontrollbohrschablone.

Abb. 4: Befestigte Konstruktionsdurchsicht am C-Arm mit Röntgenbild im anterior-posterioren sowie lateralen Strahlengang................................20

Abb. 5: C: Montierter SpineAssist ${ }^{\circledR}$ mit befestigtem Auslegearm und eingeführter Bohrhülse D: Postoperative Nähte....

Abb. 6: $\quad$ Grad 1-4 der Pedikelschraubenfehllage. .23

Abb. 7: $\quad$ Messung des Ho-Winkels .24

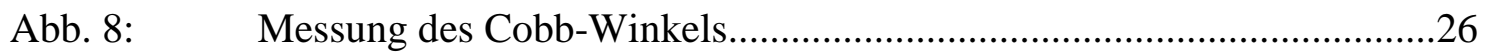

Abb. 9: $\quad$ Verteilung der Schrauben auf Wirbelkörper...........................................32

Abb. 10: Verteilung der Schrauben mit Fehllage auf Wirbelkörper........................34

Abb 11: $\quad$ Verteilung korrekter und fehlplatzierter Schrauben bei männlichen und weiblichen Patienten.

Abb. 12: Verteilung korrekter und fehlplatzierter Schrauben bei BMI < und $\geq 30$ $\mathrm{kg} / \mathrm{m}^{2}$. .36

Abb. 13: Verteilung korrekter und fehlplatzierter Pedikelschrauben auf durchschnittliche Hounsfieldeinheit $<100$ HE und $\geq$ 100HE. .38

Abb. 14: Verteilung korrekter und fehlplatzierter Schrauben bei gesunden Patienten und Patienten mit einer Spondylolisthesis Grad I und II nach Meyerding. .39 


\section{Tabellenverzeichnis}

Tabelle 1: Anzahl implantierter Schrauben pro Patient 31

Tabelle 2: Verteilung der Anzahl an Schraubenfehllagen pro Patient auf Anzahl implantierter Schrauben absolut............................................................33

Tabelle 3: Verteilung des Cobb-Winkels nach korrekter und fehlpositionierter Schraubenlage. 40

Tabelle 4: Verteilung korrekt und fehlplatzierter Schrauben auf Anzahl fusionierter Wirbel. 40

Tabelle 5: Verteilung und Signifikanz von demographischen und winkelassoziierten Parametern sowie Spondylolisthesis und HE. .42

Tabelle 6: Uni- und multivariable logistische Regressionsanalyse .43 


\section{Abkürzungsverzeichnis}

CT: $\quad$ Computertomographie

BWS: $\quad$ Brustwirbelsäule

BWK: Brustwirbelkörper

LWS: Lendenwirbelsäule

LWK: Lendenwirbelkörper

SWK: $\quad$ Sakralwirbelkörper

OP: Operation

HE: $\quad$ Hounsfieldeinheit

3D: dreidimensional

LED: $\quad$ light emitting diods

a.p.: anterior-posterior

WHO: World Health Organization

MD: $\quad$ Meyerding

BMI: $\quad$ Body-Mass-Index

CI: Koinfidenzintervall

Proc.: Processus

Procc.: Processi

PLIF: $\quad$ Posteriore Lumbale Interbody Fusion

DEXA: dual-energy $x$-ray absorptiometry 


\section{Einleitung}

\subsection{Zielsetzung und Aufgabenstellung der Arbeit}

Als Versorgungsstandard der Wirbelsäulenstabilisierung gilt der Einsatz von Pedikelschrauben. Konventionelle Operationstechniken können diese Stabilisierung zwar bewirken, sie weisen aber ein hohes Maß an Fehllagen von bis zu $40 \%$ auf (Jerosch et al. 1992). Folgen solcher Fehllagen können, neben der reduzierter Stabilität, Spinalwurzelläsionen und sensomotorische Defizite sein (Lonstein et al. 1999; Weinstein et al. 1992; West et al. 1991). Daher hat das korrekte Platzieren von Pedikelschrauben höchste Priorität.

Die Ende des 20. Jahrhunderts eingeführte navigationsassistierte Pedikelverschraubung konnte die Fehllageraten deutlich reduzieren, wenngleich auch bei diesem Verfahren weiterhin missplatzierte Schrauben auftraten (Amiot et al. 2000; Laine et al. 1997b; Seller et al. 2005).

Die Verringerung der Fehllagerate bei roboterassistierter Operationstechnik konnte in vorausgegangenen Studien bereits belegt werden (Hu et al. 2013; Kantelhardt et al. 2011). Dennoch besteht weiterhin das Problem von Fehllagen, wobei die bisherige Datenauswertung bezüglich der Ursachen dieser Ungenauigkeit nicht zufriedenstellend ist. Die vorliegende Arbeit soll die Zusammenhänge zwischen roboterassistierter Pedikelschraubenimplantation und möglichen Fehlerquellen analysieren.

Diese Arbeit befasst sich mit der retrospektiven Datenauswertung von 182 Patienten, die zwischen 2008 und 2012 eine roboterassistierte Pedikelschraubenimplantation der thorakolumbalen Wirbelsäule an der Neurochirurgischen Klinik der Universitätsmedizin Göttingen erhielten. Die Lageposition von Pedikelschrauben wurde anhand postoperativer CT-Bilder beurteilt und mithilfe eines Gradings eingeteilt. Zusätzlich wurden Datensätze individueller Patientenparameter ausgewertet.

Ziel der Arbeit ist es, mögliche Zusammenhänge zwischen den Fehllagen der Pedikelschrauben bei roboterassistierter Technik und den spezifischen Parametern des Patientenkollektivs herauszufiltern. Des Weiteren soll die Akkuratesse und die Reliabilität des Robotersystems analysiert werden. Die Detektion von Faktoren, die sich negativ auf das Operationsergebnis auswirken, könnte den Einsatz des Roboters spezifizieren und die Behebung von Fehlerquellen ermöglichen. 


\subsection{Klinische Bedeutung der Pedikelschraubenfehlplatzierung}

Das korrekte Einbringen der Pedikelschrauben ist für das Vermeiden von intra- und postoperativen Komplikationen entscheidend. Neben allgemeinen Komplikationen von Stabilisierungsoperationen wie Blutungen, Wundinfektionen, Liquorfisteln, Schraubenlockerung oder Implantatbrüchen kann das fehlerhafte Einbringen von Pedikelschrauben zur Verletzung von Nachbarstrukturen führen.

Je nach Wirbelsäulenabschnitt kann eine laterale Abweichung aufgrund der anatomischen Verhältnisse zur Verletzung von Aorta, Intercostalgefäßen, Truncus sympathicus und Lunge führen (Vaccaro et al. 1995). Ein mediales Abweichen ist besonders riskant, da hierbei das Eintreten der Pedikelschraube in den Spinalkanal möglich ist. Neben Liquorleckagen sind auch Verletzungen des Rückenmarks und der austretenden Spinalnerven möglich. Die von Gertzbein und Robbins 1990 definierte sichere Zone besagt, dass ein Eindringen der Pedikelschrauben in den Spinalkanal von 0-4 mm toleriert werden kann, da hierbei das Risiko einer Verletzung intraduraler Strukturen sehr gering ist. Insbesondere bei voroperierten Patienten im Bereich der Wirbelsäule ist die Komplikationsrate höher (Esses et al. 1993). Trotz geringer Raten an ernsthaften Komplikationen, wie direkte oder indirekte Wurzelläsionen, muss das höchste Maß an Präzision immer angestrebt werden (Matsuzaki et al. 1990; Rivet et al. 2004). Ein Abweichen der Pedikelschrauben sollte daher unter 4 mm liegen .

\subsection{Entwicklung und Ergebnisse der nicht-navigationsassistierten Spondylodese}

Viele Pathologien, wie beispielsweise Wirbelkörperfrakturen, Degenerationen (wie die Spondylolisthesis), Entzündungen (z. B. Spondylodiszitis) oder tumoröse Erkrankungen der Wirbelsäule, können eine Instabilität verursachen und bergen dadurch das Risiko für neurologische Komplikationen. Die Spondylodese, eine operative, segmentale Versteifung der Wirbelsäule, kann die Stabilität und Aufrechtstellung der Wirbelsäule wiederherstellen. Die Indikation dafür ist beim Auftreten von Paresen oder bestehender Instabilität bzw. Schmerz trotz Ausschöpfung der konservativen Therapien gegeben (Magerl 1980). Weltweit stellt die transpedikuläre dorsale Verschraubung dabei heutzutage ein Standardverfahren dar. 
Während die transpedikuläre Verschraubung erstmals 1944 durch King beschrieben wurde, machten Roy-Camille et al. (1986) Mitte der 80er Jahren den Einsatz von Pedikelschrauben populär. Dick et al. entwickelten dieses System 1985 weiter zum Fixateur interne. Zu diesem Zeitpunkt standen mehrere alternative Möglichkeiten, wie beispielsweise die Stellschraubenfixation nach Magerl oder die HarringtonDistraktionsstäbe, zur Verfügung (Gertzbein et al. 1982; Magerl 1980). Der Einsatz von Pedikelschrauben hatte jedoch den Vorteil, dass im Vergleich $\mathrm{zu}$ den alternativen Methoden eine erhöhte Winkelstabilität erzielt werden konnte (Dick et al. 1985; Dickman et al. 1992; Jerosch et al. 1992). Das Prinzip bestand dabei in einer Verbindung der eingesetzten Pedikelschrauben mit einer Lochplatte, die als Längsträger diente. Nachteil des Einsatzes von Lochplatten war, dass eine ausreichende Stabilität nur erzielt werden konnte, wenn mindestens zwei Wirbel ober- und zwei Wirbel unterhalb der Pathologie verschraubt wurden und sich damit die Spondylodese und damit die Immobilität der Wirbesäule über einen längeren Abschnitt erstreckte (RoyCamille et al. 1986).

Daher wurde in den folgenden Jahren eine verbesserte Methodik entwickelt, die anstelle der Lochplatten ein Stabsystem vorsah. Die Pedikelschrauben benachbarter Wirbel wurden über longitudinal angelegte Stäbe miteinander verbunden Durch die Vier-PunktFixation der Pedikelschrauben in Kombination mit Verbindungsstäben mussten nun lediglich die direkten Nachbarwirbel verschraubt werden, um eine ausreichende Stabilität zu garantieren (Dick et al. 1985; Dickman et al. 1992). Ein weiterer Vorteil neben der Stabilisierung war die zusätzliche Möglichkeit der Distraktion, Kompression oder Reposition der betroffenen Wirbelsäulensegmente und eine Zunahme der Winkelstabilität (Dick et al. 1985; Jerosch et al. 1992).

Um eine optimale Schraubenlage zu erzielen, kristallisierten sich zudem zwei Schrauben-Eintrittspunkte in den Pedikel heraus: Roy-Camille et al. (1986) bestimmten den Eintrittspunkt durch zwei sich kreuzende Linien. Die Vertikale verlief als Verlängerung der Facettengelenke, die Horizontale verlief mittig durch die Procc. transversi des Wirbels. Weinstein et al. ergänzten 1988 dies durch einen weiteren Eintrittspunkt, der sich lateral-kaudal des oberen Facettengelenks des Wirbels befand und im Bereich der LWS bessere Ergebnisse als der erstgenannte Eintrittspunkt erzielte (Weinstein et al. 1988).

Intraoperativ bestand jedoch die Problematik, dass zur Berechnung der idealen Bohrbahn in den Pedikel lediglich anatomische Landmarken sowie eine 
Röntgenaufnahme vor und nach dem Bohrvorgang dienten, wodurch der Operateur den Bohrvorgang nicht kontinuierlich verfolgen konnte.

Daher wurden ab den 80er Jahren intraoperativ Bildwandler eingesetzt, die die Möglichkeit eröffneten, die Schraubenimplantation unter Durchleuchtungskontrolle der Wirbel durchzuführen.

Die verbesserte Bildgebung ermöglichte nun auch eine genauere postoperative Analyse der Pedikelschraubenlage, die durch Bilder einer CT (Computertomographie) erfolgte. Es zeigte sich jedoch, dass ein hoher Prozentanteil der implantierten Schrauben Fehllagen aufwiesen: Jerosch et al. (1992) stellten eine Abweichung der Schraubenlage innerhalb des Pedikels in 39,9\% fest. Sie verwiesen dabei auf die Unfähigkeit des CArms, die dreidimensionale Komplexität des Pedikels zu erfassen und machten diese eingeschränkte Sicht hauptverantwortlich für die hohe Fehllagerate. Ähnliche Studien verzeichneten Fehllageraten von 19-37 \% und untermauerten damit die Notwendigkeit, das Operationsverfahren zu optimieren (Gertzbein und Robbins 1990; Laine et al. 1997a; Weinstein et al. 1988). Anzumerken ist hierbei, dass die genannten Analysen mit Nativ-Röntgen- oder CT-Bildern durchgeführt wurden, wobei die Fehllageanalyse mittels CT-Bildern der Analyse von Nativ-Röntgenbildern deutlich überlegen ist (Farber et al. 1995).

\subsection{Entwicklung und Ergebnisse der navigationsassistierten Spondylodese}

Die Innovationen im Bereich der Computertechnologie verbesserten schließlich auch das operative Verfahren der Spondylodese. Die eingesetzten Technologien waren im Detail zwar verschieden, hatten jedoch bestimmte Komponenten gemein, weshalb ihr allgemeiner Einsatz als navigations-/ computerassistiert oder image-guided (bildgeführt) bezeichnet wurde (Amiot et al. 2000; Nolte et al. 1995 b; Seller et al. 2005). Das Prinzip der Navigationsassistenz soll hier kurz beschrieben werden:

Präoperativ wurde eine CT des betroffenen Wirbelsäulenabschnitts angefertigt, die über eine Software zu dreidimensionalen Bildern verarbeitet wurde. Zusätzlich wurde ein axialer, sagittaler und koronarer Schnitt auf dem Computermonitor angezeigt. Dem Operateur war es so bereits präoperativ möglich, sich genaue Kenntnisse über die 
vorliegende Anatomie anzueignen. Der Eintrittspunkt sowie der Verlauf der Trajektorie für die Pedikelschraube durch den Knochen konnte dabei ebenfalls präoperativ auf den Bildern markiert werden.

Intraoperativ wurde die Navigation über mehrere Systemkomponenten gesteuert. Zu Beginn der OP (Operation) diente eine Röntgenaufnahme mittels C-Arm zur Orientierung am Patienten.

Während der Operation konnte mittels mobiler Infrarotkamera die Position des Instrumentariums und einer Referenzbasis, die mit LEDs (light emitting diods) ausgestattet waren, detektiert werden. Diese Informationen wurden an eine Rechnereinheit gesendet, wodurch eine exakte Abgleichung der prä- und intraoperativen Bilder möglich wurde. Der Operateur konnte dadurch am Monitor die Bohrrichtung und Eindringtiefe der Instrumente verfolgen und zeitgleich die Pedikelschraubenplatzierung durchführen (Arand et al. 2001; Seller et al. 2005, Schnake 2002).

Bereits 1995 wurde die navigationsassistierte Schraubenimplantation in vitro untersucht und eine Abweichung der korrekten Schraubenlage in nur $10 \%$ festgestellt (Nolte et al. 1995 a; Nolte et al. 1995 b). Die erhoffte Verbesserung durch die navigationsassistierte Chirurgie wurde in vivo ebenfalls in mehreren Studien bewiesen:

Amiot et al. (2000) verglichen die postoperative Schraubenlage von 544 konventionell implantierten Schrauben mit 294 navigationsassistierten Schraubenlagen. Während bei der konventionellen Implantation $15 \%$ der Schrauben eine Fehllage aufwiesen, waren es bei der navigationsassistierten Gruppe nur $5 \%$. Hinzu kam, dass in allen Fällen der navigationsassistierten Fehllagen die Abweichung 1-2 mm betrug, während bei der konventionellen Kontrollruppe 15 Schrauben $>2,1 \mathrm{~mm}$ von der korrekten Lage abwichen. In zwei Fällen wurden fehllagebedingte neurologische Defizite nachgewiesen, die eine Reoperation nötig machten. In der navigationsassistierten Gruppe lag keine Indikation zur Reoperation vor, lediglich zwei Patienten klagten über persistierende Schmerzen, die unter konservativer Therapie erfolgreich behandelt werden konnten.

Ähnliche Ergebnisse wiesen auch Laine et al. (2000) bei einem Vergleich beider Methoden nach: Lediglich 4,6\% der navigationsassistierten Pedikelschrauben zeigten eine Fehllage, wobei diese stets unter $4 \mathrm{~mm}$ lag. Die konventionelle Kontrollgruppe wies $13,4 \%$ Fehllagen auf, darunter $1,45 \%$ mit einer Abweichung $>4 \mathrm{~mm}$. Weitere 
Studien zeigten bei navigationsassistierter Chirurgie Pedikelschraubenfehllagen in 1,8$8 \%$ und bewiesen damit den Vorteil der Navigationschirurgie (Kotani et al. 2007; Merloz et al. 1998; Rajasekaran et al. 2007).

Neben der verbesserten Präzision der Schraubenimplantation hatte die Navigationsassistenz noch weitere Vorteile: Rajasekaran et al. (2007) untersuchten den Einsatz des C-Arms während konventioneller und navigierter Operationstechnik. Während der C-Arm beim konventionellen Verfahren 1,5 0,25 mal pro Schraubenimplantation genutzt wurde, reichte beim Einsatz des Navigationssystems eine Bildaufnahme zur Orientierung für die Implantation von durchschnittlich 11,4 Schrauben. Daraus kann geschlossen werden, dass die intraoperative Strahlenbelastung des Personals und des Patienten durch den C-Arm deutlich gesenkt werden konnte.

Kosmopoulos und Schizas veröffentlichten 2007 eine Metaanalyse, in der sie die Ergebnisse konventioneller und navigationsassistierte Pedikelschraubenimplantation aus 130 Studien miteinander verglichen. Sie untersuchten dabei in vivo platzierte sowie in Kadavern platzierte Schrauben. Die Analyse ergab einen höheren medianen Anteil korrekt platzierter Schrauben bei navigationsassistierter Chirurgie $(95,2 \%)$ für in vivo platzierte Schrauben. Konventionell platzierte Pedikelschrauben waren im Median in nur 90,3\% korrekt verschraubt worden. Für die Einbringung von Pedikelschrauben im thorakalen Wirbelsäulenbereich konnte jedoch in vivo sowie bei Kadavern kein Vorteil der navigationsassistierten gegenüber der konventionellen Technik gezeigt werden. Dies führten sie zum einem auf den Mangel an vorhandenen Studien, die lediglich die Genauigkeit thorakal platzierter Schrauben untersuchte, zurück. Zum anderen wiesen sie auf den kleineren Pedikeldurchmessers der thorakalen Wirbel hin, wodurch der Zugang und Gebrauch der Operationsinstrumente erschwert wird.

Die verbesserte Genauigkeit der Pedikelschraubenpositionierung bei navigationsassistierter Pedikelverschraubung konnte in weiteren Meta-Analysen ebenfalls bestätigt werden (Tian und Xu 2009; Verma et al. 2010). Verma et al. (2010) wiesen in der Analyse von 23 Studien, die die Fehllagen in navigationsassistierter Pedikelverschraubung untersuchten, einen Anteil korrekt platzierter Schrauben in 93,3\% nach. In 14 Studien wurde zudem eine Kontrollgruppe mit konventioneller Insertion der Schrauben analysiert, bei denen in nur 84,7 \% Pedikelschrauben korrekt platziert wurden. Das relative Risiko einer Fehllage betrug 1,12 (95-Koinfidenzintervall (CI): 1,09-1,15) für die konventionelle Pedikelverschraubung gegenüber der 
Navigationsassistenz. Zusätzlich wurde in dieser Meta-Analyse das funktionelle Outcome bei navigationsassistierter und konventioneller Technik untersucht. Es konnte jedoch kein signifikanter Vorteil der navigationsassistierten Technik bezüglich neurologischer Kompikationen, Schmerzlinderung oder Fusionsrate gefunden werden. Hierbei wurde jedoch aufgezeigt, dass eine signifikante Aussage aufgrund mangelnder Datenlage nicht möglich war.

Navigationsassistierte Pedikelverschraubung zeigte sich auch bei spinal voroperierten Patienten als vorteilhaft. Die Problematik bei dieser Patientengruppe besteht in der erschwerten Orientierung, da anatomische Landmarken hier meist teilweise oder vollständig fehlen. Lim et al. (2005) untersuchten 35 voroperierte Patienten, die eine navigationsassistierte Revision erhielten. Fünf der 122 eingesetzten Schrauben wiesen eine Fehllage auf, womit $96 \%$ aller Schrauben korrekt lagen. Keine der fehlliegenden Schrauben führte zu neurologischen Defiziten oder notwendiger Revision aufgrund der Fehllage.

Auch Austin et al. untersuchten 2002 diese Problematik, in dem sie in einer Kadaverstudie Knochenzement entlang der dorsalen Wirbelsäulenoberfläche verteilten und so fusionierte Wirbelsäulenabschnitte imitierten. Sie untersuchten die Unterschiede zwischen computer- und Röntgen-assistierter Technik mit der offenen Laminoforaminotomie-Technik, die ohne jegliche Bildgebung auskam. 21,43\% der Pedikelschrauben, die mit offener OP-Technik am fusionierten Modell verschraubt wurden, wiesen Fehllagen auf. Der Anteil an Fehlagen im Fusionsmodell war bei einfacher Röntgenbildgebung $8,33 \%$, in der navigationsassistierten wurden keine Fehllagen gefunden.

Nachteile der Navigation waren neben den hohen Anschaffungskosten der zeitaufwendige Registrierungsprozess und die dabei notwendige direkte Sicht auf das Operationsfeld. Ebenso erhielt der Patient aufgrund des präoperativen CTs eine höhere Gesamtstrahlendosis. Dies ist jedoch unter Berücksichtigung des größeren Anteils an korrekter Schraubenlage und damit einem geringeren Risiko für damit verbundene Komplikationen zu bewerten (Merloz et al. 1998).

Problematiken zeigten sich auch bei voroperierten Patienten, denen posteriore Anteile, wie die Laminae arcus vertebrae oder der Proc. spinosus des Wirbels, fehlten, wodurch die Oberflächenregistrierung erschwert wurde. Ebenso stellten eine teilweise insuffiziente Befestigung der Referenzbasis sowie Funktionseinschränkungen der LEDs ein Hindernis für die navigationsassistierte Chirurgie dar (Laine et al. 1997 b). 
Die weiterhin bestehenden Fehllageraten führten Arand et al. (2001) und Lim et al. (2005) darauf zurück, dass während der Implantation der Pedikelschrauben keine Führung der Verschraubung bestand. Die Einbringung der Pedikelschraube wurde weiterhin frei und manuell durchgeführt, wodurch auch bei vorgegebener Bohrbahn immer noch eine falsche Platzierung durch den Operateur möglich war. Zudem zeigte sich bei Arand et al. (2001) ein Anstieg des Anteil korrekt platzierter Schrauben von $72 \%$ im ersten halben Jahr der Anwendung auf $88 \%$ im zweiten Halbjahr, was auf eine Lernkurve für das Verfahren hindeutet. Er unterstrich damit die Abhängigkeit des postoperativen Ergebnisses von der Einarbeitung und genauen Aufklärung des Operateurs für das Navigationssystem.

Mit der Einführung von Robotertechnik zu Beginn des 21. Jahrhunderts versprach man sich die Lösung dieser Problematiken.

\subsection{Besondere Patientengruppen bei Pedikelschraubeninsertion}

Skoliotische Veränderungen der Wirbelsäule können ohne adäquate Therapie zu schmerzhafter Osteoarthose und Radikulopathien führen (Bradford et al. 1999). Eine adäquate Therapie ist daher unumgänglich. Eine operative Korrektur der Skoliose sollte jedoch nur bei schwerer Krümmung der Wirbelsäule $\left(>40^{\circ}\right)$ und einer raschen Progredienz der Deformität und Symptome vorgenommen werden. Nach Möglichkeit sind die konservativen Therapien insbesondere bei jungen Patienten auszuschöpfen und eine operative Korrektur vor dem 10.-12. Lebensjahr nicht anzustreben (Rüther und Lohmann 2014). Die Schwierigkeit hierbei ist, dass die Ansicht des Proc. spinosus und anderer dorsaler Wirbelanteile kaum Informationen über die Lage der ventralen Anteile des Wirbels hergibt. Das Risiko einer Pedikelschraubenfehllage bei konventioneller Operationstechnik ist bei Patienten mit degenerativer Skoliose deutlich höher, als bei Patienten ohne diese Pathologie (Yoshii et al. 2015). Navigations- und roboterassistierte Techniken bieten daher einen großen Vorteil gegenüber konventionellen Techniken, da sie genau diese Informationen durch ihre Bildgebung prä- und intraoperativ liefern können (Devito et al. 2010; Jin et al. 2015).

Eine weitere Patientengruppe, die besonderer Beachtung bei der Planung der Pedikelverschraubung verdient, sind Patienten mit Spondylolisthesis. 
Die Anatomie von Patienten mit einer spondolytischen Spondylolisthesis ist im Vergleich zum Gesunden verändert. Die Länge des Pedikels im fünften Lendenwirbelkörper (LWK) ist größer und die Höhe des Wirbelkörpers kleiner als bei Patienten ohne Spondylolyse (Matthews et al. 2015). Dies ist bei der Auswahl der zu implantierenden Pedikelschraube zu berïcksichtigen.

Bei Patienten mit degenerativer Spondylolisthesis steigt mit zunehmendem Alter und abnehmender Knochenmineraldichte das Risiko einer Stabilitätsabnahme der Spondylodese im Langzeitverlauf (Wu et al. 2008). Die Posteriore Lumbale Interbody Fusion (PLIF) erzielt im Verlauf eine bessere Stabilität als die alleinige Pedikelverschraubung (La Rosa et al. 2003). Bei der genannten Interbody-Fusion wird die Bandscheibe zwischen den Wirbelkörpern partiell oder vollständig entfernt und anstelle dessen ein Titan- oder Peekkorb mit körpereigenem Knochen oder synthetischem Knochenersatzmaterial eingebracht. Im weiteren Verlauf kommt es dann zur Verknöcherung dieses Segements und zusätzlicher Stabilisierung. Neben der PLIF gibt es auch transforaminale, laterale oder anteriore Zugangswege. Aufgrund der zusätzlichen Stabilisierung kann bei der genannten Patientengruppe eine zusätzliche Interbody-Fusion sinnvoll sein, was jedoch auch größere Ansprüche an die Operationsbedingungen stellt.

\subsection{Perkutane versus offene Operationstechnik und ihr Stellenwert bei navigations- und roboterassistierter Pedikelverschraubung}

Perkutane Operationstechniken sind in den letzten Jahren zunehmend häufiger geworden, was nicht zuletzt durch die zunehmende Anzahl an Studien über diese Operationstechnik unterstrichen wird.

Erste Ansätze einer perkutanen Verschraubung wurden bereits 1980 durch Magerl vorgestellt. Wiesner et al. (2000) analysierten die perkutane Verschraubung im lumbalen Wirbelsäulenbereich bei konventioneller Operationstechnik: 6,6\% der 408 platzierten Pedikelsschrauben wiesen Fehllagen auf. Der größte Anteil missplatzierter Schrauben befand sich im ersten Sakralwirbel (12\% aller Fehllagen). Begründet wurde dies mit der Nähe des Os sacrum zum hervorstehendem Beckenkamm, wodurch eine mediale Abweichung während der Pedikelschraubeninsertion provoziert werden würde. 
Postoperativ wurden in zwei Fällen Wurzelverletzungen des Spinalnervs festgestellt, wovon einer auf eine fehlliegende Pedikelschraube zurückgeführt werden konnte.

Schizas et al. (2007) zeigten bei konventioneller Operationstechnik eine deutlich höhere Fehllagerate bei perkutaner Verschraubung: die postoperative CT-Kontrolle von 60 implantierten Pedikelschrauben bei 15 Patienten wies in der axialen Ansicht in $23 \%$ Fehllagen, in der koronaren Ansicht in 30 \% Fehllagen auf. Der Anteil an Fehllagen, die mehr als 6 mm von der korrekten Lage abwichen, war in beiden Ansichten 3,3\%. Ein Patient entwickelte aufgrund einer Fehllage ein neurologisches Defizit im Bereich der Spinalwurzel S1.

Auch perkutane, konventionelle Operationstechniken, in denen unterschiedliche Eintrittspunkte nach Magerl oder nach Wiesner erfolgten, wiesen Fehllagen in 8-13\% auf (Wiesner et al. 1999).

Grass et al. (2006) konnten ebenfalls geringere Fehllageraten bei perkutaner Verschraubung (6\%) im Vergleich zur offenen Technik feststellen (12\%).

Wie schon bereits im Abschnitt 1.4 beschrieben, war bei navigationsassistierter (nichtperkutaner) Technik der Anteil fehlplatzierter Schrauben kleiner: zwischen 2-6,7 \% der Schrauben wiesen Fehllagen auf, lediglich Merloz et al. (1998) fanden in 8 \% Fehllagen (Amiot et al. 2000; Kosmopoulos and Schizas,2007; Kotani et al. 2007; Laine et al. 2000, 1997b; Merloz et al. 1998; Rajasekaran et al. 2007).

Die Kombination von navigationsassistierter und perkutaner Technik untersuchten Holly und Foley 2003: 94 Pedikelschrauben wurden mithilfe der Navigationsassistenz perkutan verschraubt, 94,7\% wurden dabei korrekt platziert. $100 \%$ der lumbalen Pedikelschrauben lagen korrekt, thorakal waren es $92 \%$. Dies stand im Einklang mit den Fehllageraten bei offener Navigationsassistenz. Man kann daraus schlussfolgern, dass die perkutane Verschraubung für die Genauigkeit der Schraubenlage weniger von Bedeutung ist als die Verwendung navigationsassistierter Chirurgie.

Der große Stellenwert perkutaner Pedikelschraubenimplantation in der heutigen Neurochirurgie ist dennoch berechtigt:

Denn ein Nachteil offener Operationstechniken ist ein höheres Risiko der iatrogenen Nervenläsion der medialen Äste der Rami dorsales nervi spinales (Regev et al. 2009). Eine Schädigung dieser Nerven im Bereich der autochthonen Rückenmuskulatur kann im Verlauf zur Muskelatrophie führen, welche die Enstehung eines failed back surgery syndrome begünstigen kann (Sihvonen et al. 1993). Das Risiko einer 
Schädigung des Musculus multifidus, der als stärkster Extensor der autochthonen Rückenmuskulatur gilt, wird bei perkutanem Zugangsweg signifikant reduziert (Grass et al. 2006).

Zusätzlich ist die postoperative Wundfläche beim perkutanen Zugang kleinflächiger und der Blutverlust geringer (Lehmann et al. 2008; Lieberman et al. 2012). Perkutanoperierte Patienten benötigen im Vergleich zu konventionell operierten Patienten postoperativ weniger Opiode zur adäquaten Analgesie. Zudem ist die Dauer des Krankenhausaufenthaltes kürzer und der Anteil postoperativer Komplikationen, wie Liquorlecks oder Wundinfektion, geringer (Kantelhardt et al. 2011).

Die Untersuchungen der vorliegenden Arbeit befassen sich jedoch mit der roboterassistierten Pedikelverschraubung. Für das Ergebnis korrekt platzierter Schrauben durch den SpineAssist ${ }^{\circledR}$ ist es irrelevant, ob der operative Zugang perkutan oder offen erfolgt (Kantelhardt et al. 2011). Die genannten Vorteile perkutaner Techniken bei der Verwendung des SpineAssist ${ }^{\circledR}$ sind dennoch zu berücksichtigen.

\subsection{Mazor Robotics SpineAssist ${ }^{\circledR}$ :}

Roboterassistierte Chirurgie begann in den 90er Jahren interdisziplinär an Bedeutung zu gewinnen. Ihr Einsatz sollte eine höhere Präzision bei minimalinvasiven Prozeduren ermöglichen.

Diese Arbeit befasst sich mit dem Miniatur-Roboter SpineAssist ${ }^{\circledR}$ der Firma MAZOR Surgical Technologies (Caesarea, Israel), der seit 2005 klinisch eingesetzt wird (Shoham et al. 2006).

Im Folgenden sollen sowohl der Aufbau und die Anwendung des Roboter-Systems als auch dessen bisherige Studienergebnisse dargestellt werden.

\subsubsection{Aufbau des Systems:}

Der semi-aktive Miniatur-Roboter dient als Positionierungshilfe für die Implantation von Pedikelschrauben. Seine Hauptaufgabe ist das Auffinden der optimalen Eintritssstelle und des korrekten Eintrittswinkels für die Verschraubung sowie die präzise Positionierung der Operationsinstrumente. Dadurch wird eine im Vergleich zu 
den bisher genannten Methoden präzisere Implantation der Pedikelschrauben möglich.

Das Roboter-System beinhaltet den Miniatur-Roboter SpineAssist ${ }^{\circledR}$, eine Befestigungskomponente zwischen Roboter und Wirbelsäule sowie eine eigene Planungssoftware (Abbildung 1).

Der zylindrisch geformte Roboter hat eine Größe von 50 x $80 \mathrm{~mm}$ bei einem Gewicht von $250 \mathrm{~g}$ und ist damit ein handliches Werkzeug. Die Oberseite des Roboters bietet eine Befestigungsvorrichtung für einen von drei verschiedenen Auslegearmen, welche sich in Länge und Winkelform unterscheiden. Jeder Auslegearm hat am peripheren Ende eine Öffnung, in die eine Kanüle als Führungshilfe für den intraoperativ genutzten Bohrer eingeführt werden kann. Auslenkungen des SpineAssist ${ }^{\circledR}$ sind in allen sechs Freiheitsgraden möglich.
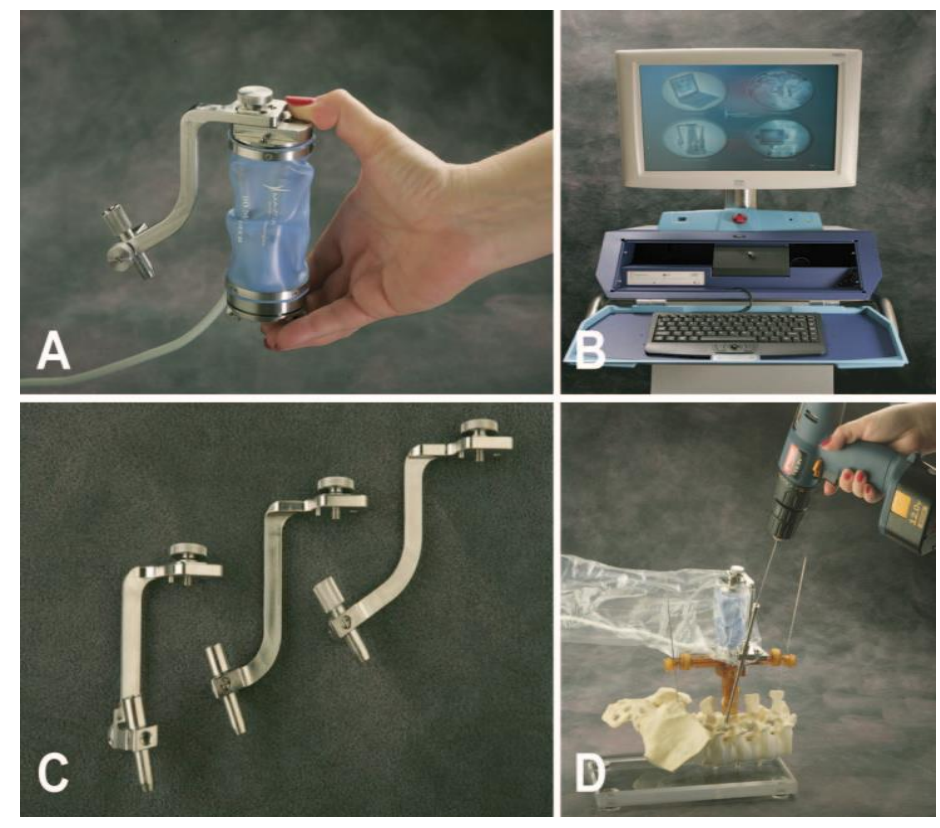

\section{Abbildung 1: SpineAssist ${ }^{\circledR}$ Roboter mit Zubehör (Liebermann et al. 2006, S. 642; die Verwendung erfolgt mit freundlicher Genehmigung der Autoren)}

Die Befestigung des Roboters an der Wirbelsäule wird durch zwei mögliche Befestigungssysteme gewährleistet:

1) In der direkten Umgebung des Zielbereichs wird an den Proc. spinosus eines Wirbels eine Klemme befestigt, die als Verbindungsstück für eine Brückenvorrichtung dient. Dafür ist eine Inzision von ca. 3-4 cm durch die paravertebrale Muskulatur nötig. Die angebrachte Brücke gliedert sich in die Abschnitte Kopf, Zentrum und Fuß. Der SpineAssist ${ }^{\circledR}$ wird auf die Brücke montiert und kann entlang dieser über drei Wirbel eingesetzt werden. 
2) Alternativ kann der sogenannte Hover-T minimally invasive frame eingesetzt werden. Dieser T-förmige Rahmen wird mit einem Kirschner-Draht am Proc. spinosus und mit zwei Steinmann-Nägeln an den Spinae iliacae posteriores superiores beidseits befestigt. Der zentrale Stab des Rahmens verläuft entlang der Wirbelsäule und bietet eine Befestigungsbasis für den SpineAssist ${ }^{\circledR}$. Entlang des Stabs kann der SpineAssist ${ }^{\circledR}$ damit an 19 verschiedenen Positionen platziert werden.

Beide Befestigungsmöglichkeiten können ohne Röntgenkontrolle angebracht werden.

Der Vorteil einer Befestigung des Roboters am Patienten liegt darin, dass atem- und manipulationsabhängige Bewegungen keinen Einfluss auf das Abstandverhältnis zwischen Roboter und Situs haben (Shoham et al. 2003).

Die sogenannte Workstation ist ein im Operationsraum stehenden Computer. Die darauf installierte Software bearbeitet präoperative CT- und intraoperative Röntgenbilder und dient damit der exakten Planung von Eintrittspunkt und Trajektorie der Pedikelschraube. Zusätzlich steuert sie den SpineAssist®, der über ein Kabel mit der Workstation verbunden ist. Der Computer verfügt außerdem über einen Touchscreen, der unter sterilen Bedingungen intraoperativ vom Operateur genutzt werden kann.

\subsubsection{State of the Art bei Verwendung des SpineAssist ${ }^{\circledR}$}

Die Vorteile des SpineAssist ${ }^{\circledR}$ gegenüber der konventionellen und computerassistierten Operationstechnik konnten in den folgenden Studien nachgewiesen werden.

Roboterassistierte Chirurgie, wie die des SpineAssist ${ }^{\circledR}$, bietet die Möglichkeit der präzisen Schraubenplatzierung, eine Reduzierung der Röntgenstrahlenexposition von Operateur und Personal sowie eine Reduzierung der Operationszeit (Kantelhardt et al. 2011; Lieberman et al. 2012). Gleichzeitig ist eine auf CT-Bildern basierte präoperative Planung durchführbar. Diese präoperative Planung ist dabei so präzise, dass es intraoperativ nur geringe Abweichungen davon bedarf, um die Schrauben akkurat zu platzieren (van Dijk et al. 2015). Robotertechnologien sind außerdem unermüdlich und unabhängig von physiologischem Hand-Tremor des Chirurgen. Die Benutzerfreundlichkeit der Software und des Registrierungsprozess vereinfacht die Einarbeitung für anzulernende Operateure. 
Für den Einsatz des SpineAssist@ werden optimalerweise nur drei Röntgenaufnahmen für die Kalibrierung der Workstation und Registrierung des Wirbelsäulenabschnitts aufgenommen.

Während der Schraubenimplantation sind weiteren Röntgenaufnahmen nicht zwingend notwendig, wodurch der Ablauf der Operation vereinfacht wird (Lieberman et al. 2006). Jedoch obliegt es dem Operateur nach Bedarf weitere Röntgen-Bilder zu veranlassen.

Die Verwendung des SpineAssist ${ }^{\circledR}$ konnte den Anteil korrekt platzierter Pedikelschrauben im Vergleich zur konventionellen Technik um $58 \%$ erhöhen (Lieberman et al. 2012). Die postoperative Schraubenlage weicht dabei durchschnittlich nur gering von der geplanten Platzierungsposition ab mit Werten zwischen 1,02 \pm 0,56 $\mathrm{mm}$ und 1,1 $\pm 0,4 \mathrm{~mm}$ (Lieberman et al. 2006, Lieberman et al. 2012).

Das Einbringen der Pedikelschrauben mithilfe des SpineAssist ${ }^{\circledR}$ minimiert den Anteil an Fehllageraten. $\mathrm{Hu}$ et al. (2013) untersuchten die Lageposition von 960 Pedikelschrauben, die in 98,9\% korrekt platziert wurden. Ähnliche Ergebnisse wurden auch in anderen Studien festgestellt, in denen der Anteil korrekt platzierter Pedikelschrauben unter Einsatz des SpineAssist ${ }^{\circledR}$ zwischen 91,7\% und 94,5\% lag (Lieberman et al. 2012; Pechlivanis et al. 2009; Roser et al. 2013). Die retrospektive Analyse von 3271 Pedikelschrauben und Kirschnerdrähten durch Devito et al. (2010) ergab eine korrekte Schraubenlage in 89,3\%. Unter Summation des Anteils, der den Pedikel mit weniger als $2 \mathrm{~mm}$ durchbrach und sich damit in der durch Gertzbein und Robbins (1990) beschriebenen sicheren Zone befand, waren 98,3\% der Pedikelschrauben korrekt platziert.

Neue Technologien verlangen das Anlernen der operierenden Chirurgen mit dem System. Für den SpineAssist ${ }^{\circledR}$ konnte gezeigt werden, dass zwar eine Lernkurve bei der Einarbeitung besteht, anzulernende Chirurgen jedoch schon nach wenigen Anwendungen konstante und adäquate Ergebnisse erzielen (Hu und Lieberman 2014; Kim et al. 2015, Schatlo et al. 2015).

In Zusammenschau dieser Ergebnisse ist die Schlussfolgerung zulässig, dass roboterassistierte Wirbelsäulenchirurgie einen Benefit für das Ergebnis der Pedikelschraubeneinbringung darstellt und ihr Einsatz damit gerechtfertigt ist.

Probleme bei der Anwendung des SpineAssists ${ }^{\circledR}$ bestehen vor allem aufgrund von Softwaredefiziten, die zu einer mangelnden Registrierung und Verarbeitung von Daten des Roboters bzw. seines Equipments führen. 
Pechlivanis et al. (2009) erhoben eine Untersuchung, in der bei zwei von 31 Patienten der Roboter aufgrund eines technischen Defekts und einer falsch gesetzten Klammer, die zur Neusetzung und Re-Registrierung führte, nicht eingestezt werden konnte. Technische Probleme beim Registierungsprozess und der intraoperativen Bohrbahnplanung führten bei $\mathrm{Hu}$ et al. (2013) zum Abbruch von 10,1\% aller Schraubeninsertionen, die anschließend manuell verschraubt werden mussten. Hierbei sind die einzelnen Gründe zu nennen: 28 Schrauben konnten bei einem Patienten aufgrund einer massiven neuromuskulären Skoliose nicht per Roboter eingesetzt werden, da das System intraoperativ nicht in der Lage war, die hochgradige Deformität adäquat $\mathrm{zu}$ registrieren und mit den präoperativen Bildern abzugleichen. Weitere Gründe waren bei jeweils einem Patienten das Unvermögen aufgrund eines massiven Weichteilmantels bzw. extrem schlechter Knochenqualität Röntgenbilder aufzunehmen, die für den Registierungsprozess geeignet gewesen wären. Ein massiver Weichtteilmantel ist bei adipösen Menschen $\mathrm{zu}$ erwarten, eine schlechte Knochenqualität ist insbesondere bei Menschen mit Osteoporose vorhanden. Auch bereits vorhandene Implantate waren bei einer Revision Störungsfaktoren für die Registrierung. Ebenso konnte bei einem Patienten mit Spondylolysis der SpineAssist ${ }^{\circledR}$ nicht eingesetzt werden, da der Bohrer keinen stabilen Ansatz im Bereich des lytischen Segments finden konnte. Lediglich in zwei Fällen war der Abbruchgrund ein rein technisches Problem.

Barzilay et al. (2006) untersuchten technische und anatomische Herausforderungen, die bei der roboterassistierten Spondylodese auftraten. In drei von 15 Patientenfällen traten Softwareprobleme der Workstation auf. Weiterhin wurde bei einem Patienten die Roboterassistenz abgebrochen, da der Eintrittswinkel in der axialen Ansicht außerhalb des Arbeitsvolumen des Roboters lag. In einem weiteren Fall konnte der Roboter nicht eingesetzt werden, da die Klammer am Proc. spinosus nicht im richtigen Winkel eingestellt werden konnte, um die gewünscht Bohrbahn mit dem SpineAssist ${ }^{\circledR}$ zu erreichen. Bei einem voroperierten Patienten wurden intraoperativ die bereits implantierten Schrauben entfernt. Aufgrund der fehlenden Prominenz der entfernten Metall-Implantate war anschließend kein Matching der präoperativen CT- und intraoperativen Röntgenbilder durch die Software mehr möglich.

Komplikationen wie vaskuläre Verletzungen und Liquorlecks treten zwar auch bei roboterassistierter Technik auf, sind aber im Vergleich zur konventionellen Technik 
weniger häufig. Dies konnten Kantelhardt et al. (2011) ebenso nachweisen wie eine geringere Revisionsnotwendigkeit aufgrund der reduzierten Fehllagerate.

Hieraus wird ersichtlich, dass die postoperativen Ergebnisse auch bei roboterassistierter Technik nicht vollständig fehlerfrei sind, auch wenn diese der konventionellen und navigationsassistierten Technik überlegen ist. Zudem sind Problematiken bekannt, die sowohl vom Roboter selbst als auch von patientenspezifischen Faktoren ausgehen.

Diese Studie dient daher der Fehlerquellendektektion. Im Abschnitt 1.5 wurden bereits die erschwerten Operationsbedingungen bei Deformitäten und Pathologien der Wirbelsäule behandelt. Ebenso wurden die Schwierigkeiten des SpineAssist ${ }^{\circledR}$, starke Skoliosewinkel der Wirbesäule zu operieren, aufgezeigt. Daher ist ein Zusammenhang zwischen Skoliose oder Olisthesis und roboterbedingter Schraubenfehllagen denkbar. Hierfür eignen sich der Cobb- und Ho-Winkel, die ein Maß für die Skoliose und Rotation der Wirbelsäule darstellen. Eine osteoporotische Knochensubstanz könnte ebenfalls eine Ursache fehlplatzierter Schrauben sein (Hu et al. 2013). Die Bildqualität von CT- und Röntgenbildern leidet durch einen zunehmenden Weichteilmantel, wie es bei adipösen Patienten der Fall ist (Uppot et al. 2007, Uppot et al. 2006). Daher sollte der Zusammenhang zwischen Adipositas und Fehllage ebenfalls untersucht werden. 


\section{Material und Methoden}

\subsection{Patientenkollektiv und klinische Datenerhebung}

Im Zeitraum zwischen Mai 2008 und Juli 2012 erhielten am Universitätsklinikum Göttingen 211 Patienten eine durch den SpineAssist ${ }^{\circledR}$ assistierte perkutane Spondylodese im thorakolumbalen Wirbelsäulenbereich. Dieser lagen ätiologisch Infektionen, maligne Prozesse, Traumata sowie degenerative Prozesse zugrunde.

Die retrospektive Untersuchung der Daten erfolgte durch die Auswertung von Patientenakten sowie prä- und postoperativen CT-Bildern. Als demographische Daten wurden Alter, Geschlecht, Körpergröße und Körpergewicht bestimmt.

\subsection{Anwendung des SpineAssist ${ }^{\circledR}$}

Der operative Einsatz des SpineAssist ${ }^{\circledR}$ lässt sich in drei Phasen gliedern:

\section{1) Präoperative Planung:}

Präoperativ wird als Bildgebung eine Computertomographie des zu operierenden Wirbelsäulenabschnitts des Patienten angefertigt. Die axialen, lateralen und anteriorposterioren Aufnahmen haben eine Schichtdicke von 0,4-1 mm. Diese CT-Bilder werden anschließend an die Workstation gesendet und bearbeitet. Alternativ kann die Bearbeitung auch an einem externen Laptop oder Computer geschehen, auf dem die Planungssoftware installiert ist. Dies hat den Vorteil, dass die Planung auch außerhalb des Operationsraums stattfinden kann. Hierbei werden die Daten nach Abschluss der Planung auf eine externe Speicherquelle, wie beispielsweise CD-ROM oder USB-Stick, kopiert und in die Workstation eingelesen.

Anhand der CT-Bilder wird über die Software ein 3D-Modell der zu verschraubenden Wirbel erzeugt. An diesem kann der Operateur den Eintrittspunkt und die Verlaufsbahn der einzubringenden Pedikelschrauben festlegen und markieren. Die virtuelle Schraube wird auf der linken Seite in oranger, auf der rechten Seite in blauer Farbe angezeigt. Gleichzeitig ist die präoperative Bestimmung der benötigten Schraubenlänge und ihres Durchmessers möglich (Abbildung 2). 
Die Software synchronisiert die CT-Bilder bei diesem Procedere automatisch. Das bedeutet, dass bei Änderung der Ansicht in einer Aufnahme alle anderen Ansichten auf die gleiche Wirbelsäulenhöhe aktualisiert werden. Somit besteht die Möglichkeit, den geplanten Schraubenverlauf in allen Ebenen zu betrachten, wodurch eine hochpräzise Planung der optimalen intrapedikulären Trajektorie erfolgt.
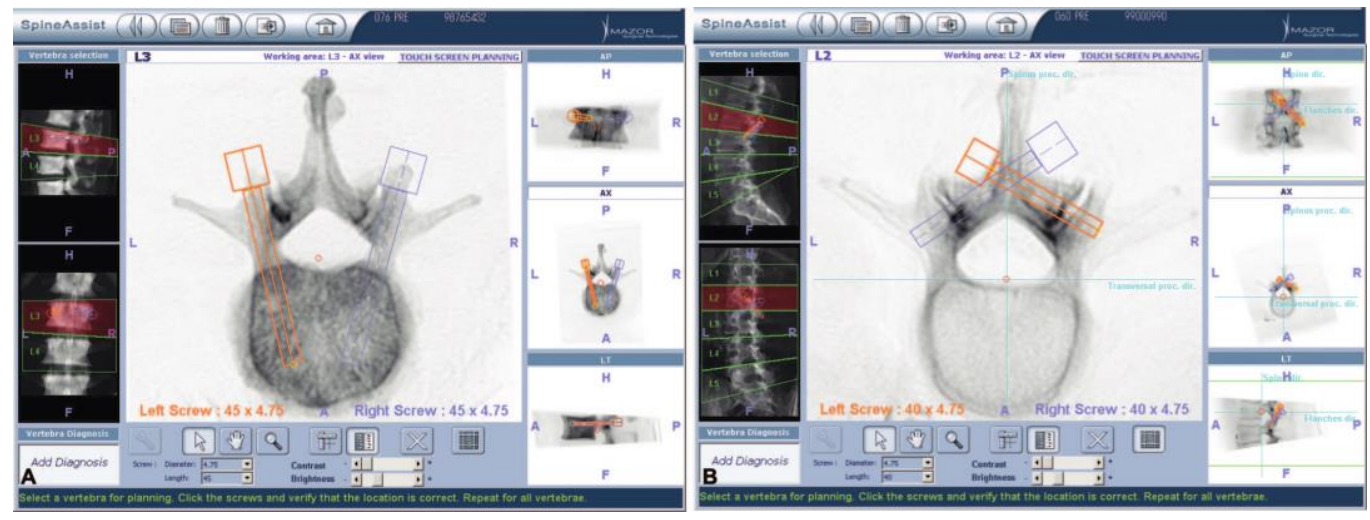

\begin{abstract}
Abbildung 2: Präoperative Planung der Verlaufsbahn der Pedikelschrauben (Liebermann et al. 2006, S. 644; die Verwendung erfolgt mit freundlicher Genehmigung der Autoren)
\end{abstract}

Im Operationssaal wird das Roboter-System vor jedem Einsatz auf die korrekte Funktionalität hin geprüft. Dafür erfolgt die Montage des SpineAssist@ auf eine Bohrschablone, die drei Kontrollöffnungen aufweist (Abbildung 3). Die Positionsdaten der Öffnungen sind in der Workstation gespeichert. Zur Überprüfung der Funktionalität wird über die Workstation der SpineAssist ${ }^{\circledR}$ zu den Kontrollöffnungen gesteuert. Sofern die Workstation mit dem Roboter fehlerfrei agiert und er die korrekte Position eingenommen hat, lässt sich über die Führungskanüle des Auslegearms ein Kirschnerdraht durch die Kontrollöffnung führen. 


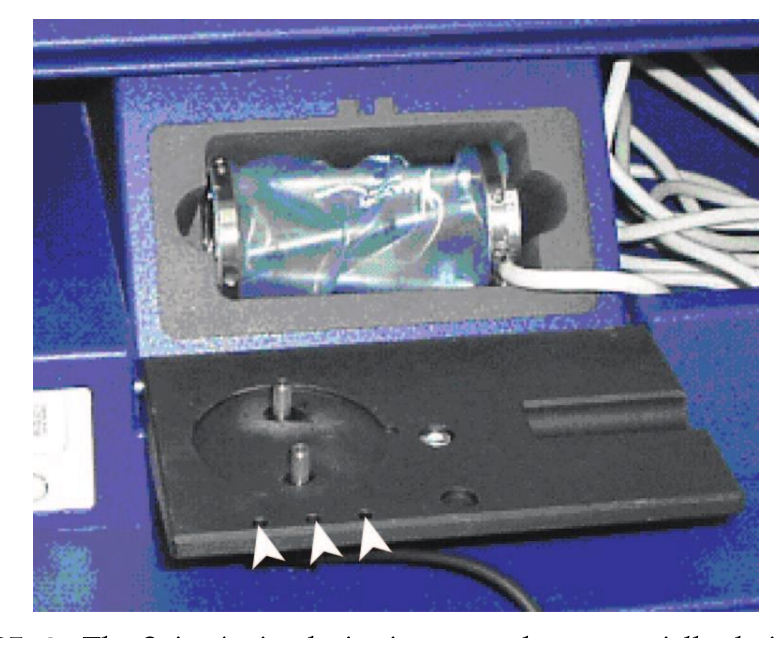

\section{Abbildung 3: Kontrollborhschablone (Liebermann et. al. 2006, S. 644; die Verwendung erfolgt mit freundlicher Genehmigung der Autoren)}

Bei erfolgreicher Kontrolle aller drei Öffnungspositionen der Bohrschablone ist von einer einwandfreien Interaktion zwischen Workstation und Roboter sowie von einer störungsfreien Funktion des Roboters auszugehen.

\section{2) Intraoperative Planung}

Während der Operation werden mittels C-Bogen Röntgenbilder benötigt, die mit den präoperativen Planungsbildern abgeglichen werden. Dafür wird eine spezielle Konstruktionsdurchsicht auf die Durchleuchtungsoberfläche des C-Bogens aufgelegt. Die Oberfläche der Durchsicht enthält kleine Metallandmarken, die in einer bestimmten Anordnung vorliegen (Abbildung 4). Dieses Muster wird von der Software registriert und erkannt und dient der Kalibrierung der Röntgenbilder sowie der Minimierung von Verzerrungen der Bilder.

Zur Kalibrierung des C-Bogens wird eine anterior-posterior-Aufnahme (a.p.) ohne ein Objekt im bestrahlten Feld durchgeführt. Auch wenn der Abstand zwischen Bildverstärker und Röntgenröhre in beiden Aufnahmen gleich ist, ist dies aufgrund der Einflüsse von elektromagnetischen Feldern (wie z. B. durch das elektronische Equipment) essentiell. Die Kalibrierung kann bereits präoperativ im OP-Saal erfolgen.

Nach Präparation des Situs wird das Klemmen- oder Hover-T-Roboter-System auf die Wirbelsäule des Patienten montiert. Erneut wird eine a.p.- und eine laterale Röntgenaufnahme durchgeführt, diesmal mit dem Wirbelsäulenzielbereich des Patienten. Anschließend wird auf die Klemme/den Rahmen ein röntgendichtes Zielgerät 
befestigt und die a.p.- und laterale Röntgenaufnahme wiederholt. Die Software registriert und verarbeitet die vier Röntgenbilder mit den präoperativen CT-Aufnahmen und den damit verbundenen Planungsdaten zu einem Bild. Das dafür benötigte Koordinatensystem errechnet sich auf Basis des röntgendichten Zielgeräts.

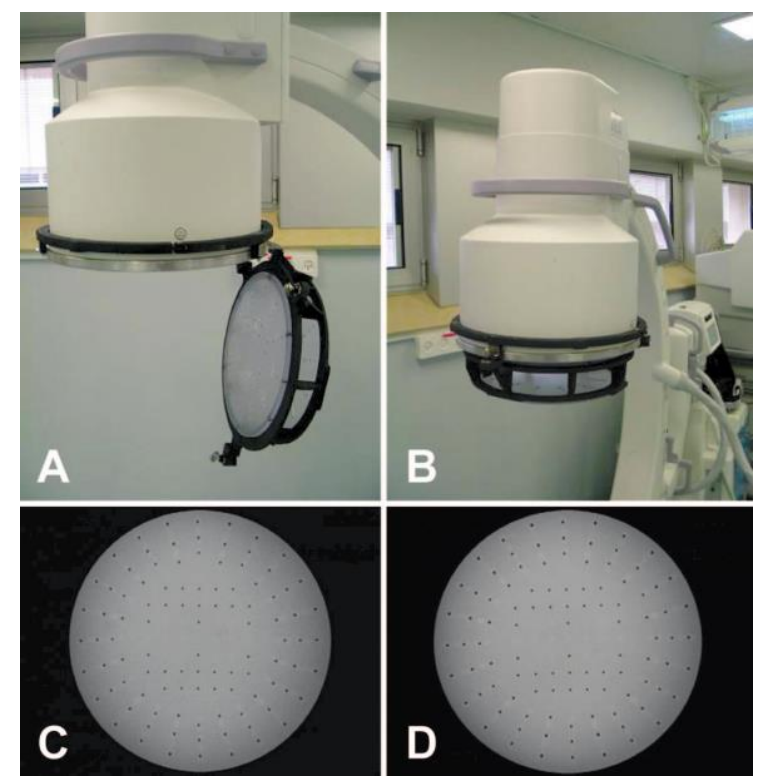

Abbildung 4: Befestigte Konstruktionsdurchsicht am C-Arm mit Röntgenbild im anterior-posterioren sowie lateralen Strahlengang (Liebermann et al. 2006, S. 645; die Verwendung erfolgt mit freundlicher Genehmigung der Autoren)

Das abgeglichene CT-Röntgen-Bild wird am Monitor zusammen mit der darüber projizierten Schraubenplatzierung angezeigt. Ist der Registrierungsprozess zu ungenau oder weicht die angezeigte Trajektorie von der korrekten Lage im Pedikel ab, blockiert die Workstation den weiteren Operationsvorgang. In diesem Fall müssen alle vier Röntgenaufnahmen wiederholt werden.

Der Operateur beurteilt nach erfolgreicher Registrierung das 3D-Bild und wählt über den Touchscreen eine geplante Schraube aus. Die Workstation berechnet daraufhin alle möglichen Positionen des Roboters, um die gewünschte Trajektorie durchführen zu können. Neben dem benötigten Auslegearm gibt sie auch die Position des SpineAssist ${ }^{\circledR}$ am Klemmen- oder Rahmensystem an. Für das Klemmensystem sind dies die Positionen entlang der Brücke (Kopf, Zentrum oder Fuß), für den Rahmen die Positionen 1-19 entlang des zentralen Stabes. 


\section{3) Pedikelschraubenimplantation}

Nachdem die geplante Trajektorie von der Workstation bestätigt wurde, wird der Roboter auf das Klemmen- oder Hover-T-System montiert. Das Verbindungskabel zwischen SpineAssist ${ }^{\circledR}$ und Workstation ist dabei mit einem sterilen Kunststoffüberzug abgedeckt. Anschließend wird der Roboter zur Zielposition gesteuert. Hat er seine Position erreicht, arretiert der Roboter in dieser Stellung. Der gewählte Auslegearm wird erst nach Erreichen der Zielposition am SpineAssist ${ }^{\circledR}$ angebracht. Durch die periphere Öffnung des Arms wird nun die Führungshülse hindurch geschoben. Der Roboter ist dabei so positioniert, dass die Führungshülse im Verlauf und Winkel zum Wirbel mit der geplanten Trajektorie übereinstimmt.

Über die Führungshülse wird eine Bohrhülse eingeführt. Über diese wird eine Stichinzision durch die Haut gesetzt und stumpf mit einem Dilatator ein Weichteilkanal bis zur Kortikalis präpariert. Über die Bohrhülse wird der Bohrer eingeführt, welcher die Kortikalis durchbohrt und eine Vertiefung in den Wirbel hinterlässt. Der Bohrer wird entfernt und ein Kirschnerdraht in die Vertiefung eingeführt (Abbildung 5). Ein Bohrer mit einem mittigen Kanal wird erneut über den Kirschnerdraht vorgeschoben und bohrt die Höhlung für die Pedikelschraube. Anschließend werden Bohrer und Kirschnerdraht entfernt und die Pedikelschraube fest verschraubt.

Dieser Vorgang beschreibt die perkutane Operationstechnik. Im Gegensatz dazu werden bei der offenen Operationstechnik die zu operierenden Wirbel vor Anlage des Befestigungssystems frei gelegt. Alle weiteren Arbeitsschritte stimmen im Verlauf jedoch mit der perkutanen Technik überein.
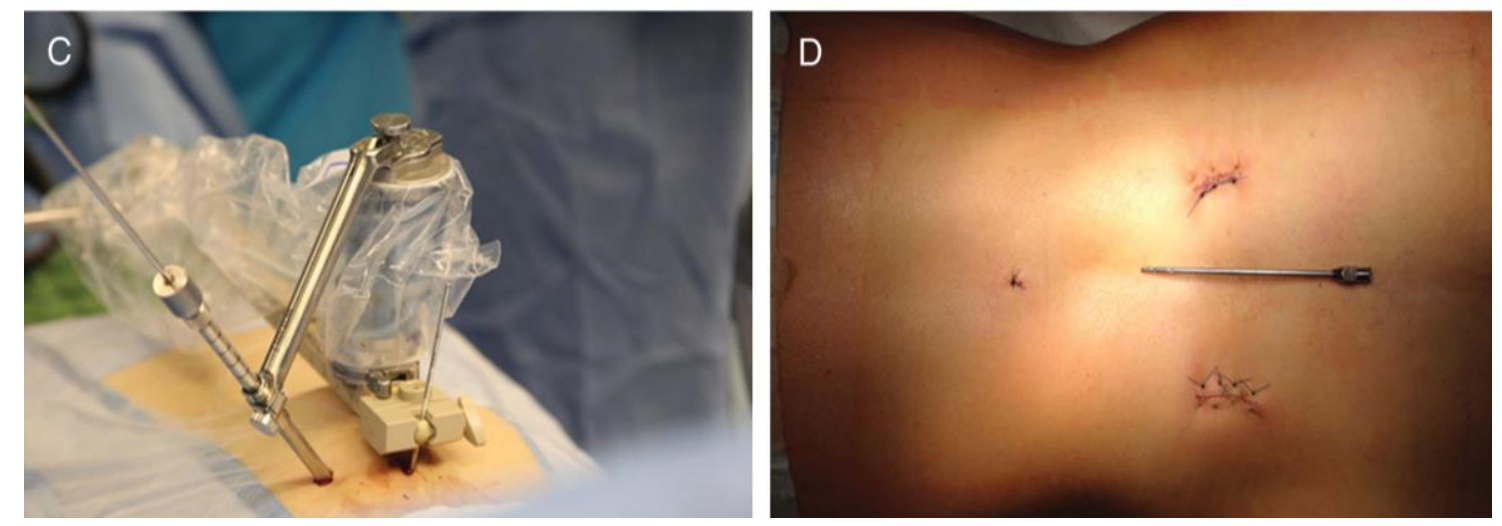

Abbildung 5: C: Montierter SpineAssist ${ }^{\circledR}$ mit befestigtem Auslegearm und eingeführter Bohrhülse D: Postoperative Nähte (Roser et al. 2013, S. A14; die Verwendung erfolgt mit freundlicher Genehmigung der Autoren) 
Durch Auswählen der folgenden Schraube am Monitor wird der Vorgang bis zur Platzierung der letzten Pedikelschraube wiederholt (Lieberman et al. 2006; Shoham et al. 2003; Sukovich et al. 2006; Togawa et al. 2007).

Anschließend erfolgt der Einsatz der Verbindungsstäbe. Dies erfolgt unabhängig vom Einsatz des SpineAssist ${ }^{\circledR}$ und kann mit einem perkutanen PathFinder ${ }^{\circledR}-$ System, der in unterschiedlicher Ausführung von verschiedenen Firmen angeboten wird, durchgeführt werden. Sofern eine offene Operationstechnik gewählt wurde, werden die Stäbe ohne ein solches System direkt eingebracht.

\subsection{Computertomographie}

Die Patienten erhielten eine prä- und postoperative Computertomographie des operierten Wirbelsäulenabschnitts. Dies diente zum einen der präoperativen Planung für die einzubringenden Schrauben, zum anderen der postoperativen Kontrolle der Schraubenlage. Außerdem wurden anhand dieser Bilder die durchschnittliche Hounsfieldeinheit (HE) sowie der Ho-Winkel gemessen.

Die Aufnahmen wurden mit dem 128-Zeiler-CT-Gerät SOMATOM® Definition AS+ der Firma Siemens (Siemens AG, Erlangen, Deutschland) durchgeführt.

Die Aquisitionsschichttdicke betrug $0,6 \mathrm{~mm}$, die daraus erstellte multiplanaren Rekonstruktionen hatten eine Schichtdicke von 2- $5 \mathrm{~mm}$. Die Röhrenspannung betrug $120 \mathrm{KV}$ und 200-300 mAs. Die Auswertung der CT-Datensätze erfolgte über die Röntgen-Betrachtungssoftware Centricity ${ }^{\mathrm{TM}}$ Enterprise Web V3.0 (GE Healthcare, USA).

\subsubsection{Klassifikation der Pedikelschraubenfehllage}

Die axialen Aufnahmen der postoperativen CT-Bilder wurden hinsichtlich der Schraubenposition im Pedikel analysiert. Abweichungen der Pedikelschrauben von der Ideallage wurden mit einer Millimeter-Skala gemessen. Für die Klassifikation der Pedikelschraubenfehllage ist in der Literatur eine Vielzahl unterschiedlicher Einteilungen beschrieben worden. Gertzbein und Robbins (1990) benutzten eine Skalierung, die die Abweichung in 2-mm-Abständen maß. Arand et al. (2001) teilten die Schraubenlage in die Gruppen A-C ein, wobei die Gruppe A keinerlei Abweichung 
aufwies. Die Gruppe B zeigte Abweichungen, die geringer als die Gewindetiefe der Schraube waren und die Gruppe $\mathrm{C}$ zeigte Abweichungen, die größer als die Gewindetiefen waren. Lediglich bei Gruppe $\mathrm{C}$ wurde eine genaue Bestimmung der Abweichung in mm angegeben.

Wiesner et al. (1999) teilten die Dislokationen ein in Schrauben, die <3 mm, 3,1- $6 \mathrm{~mm}$ und >6 mm von einer intrapedikulären Lage abwichen. Schizas et al. (2007) benutzten eine Klassifikation, bei der zusätzlich zur Klassifikation nach Wiesner noch eine Schraubenabweichung, die die Kortikalis berührt, aber nicht durchdringt, mit aufgenommen wurde.

Die Einteilung der Schraubendislokation dieser Arbeit erfolgte in Anlehnung an die Klassifikation nach Wiesner et al. (1999) und Schizas et al. (2007). Dadurch war eine präzise und dennoch übersichtliche Einteilung der Pedikelschraubenfehllage möglich:

Grad 0: Die Schraube liegt vollständig im Pedikel ohne Berührung der Kortikalis

Grad 1: Die Schraube verletzt die Kortikalis des Pedikelknochen

Grad 2: Die Schraube durchdringt die Kortikalis, die Abweichung der Schraube zur Kortikalis beträgt $<3 \mathrm{~mm}$

Grad 3: Die Schraube durchdringt die Kortikalis, die Abweichung der Schraube zur Kortikalis beträgt 3- $6 \mathrm{~mm}$

Grad 4: Die Schraube durchdringt die Kortikalis, ihre Abweichung beträgt $>6 \mathrm{~mm}$

Eine Veranschaulichung der Einteilung ist in Abbildung 6 dargestellt. Im vorliegenden Patientenkollektiv wurde keine Schraubenfehllage Grad 3 oder 4 festgestellt.

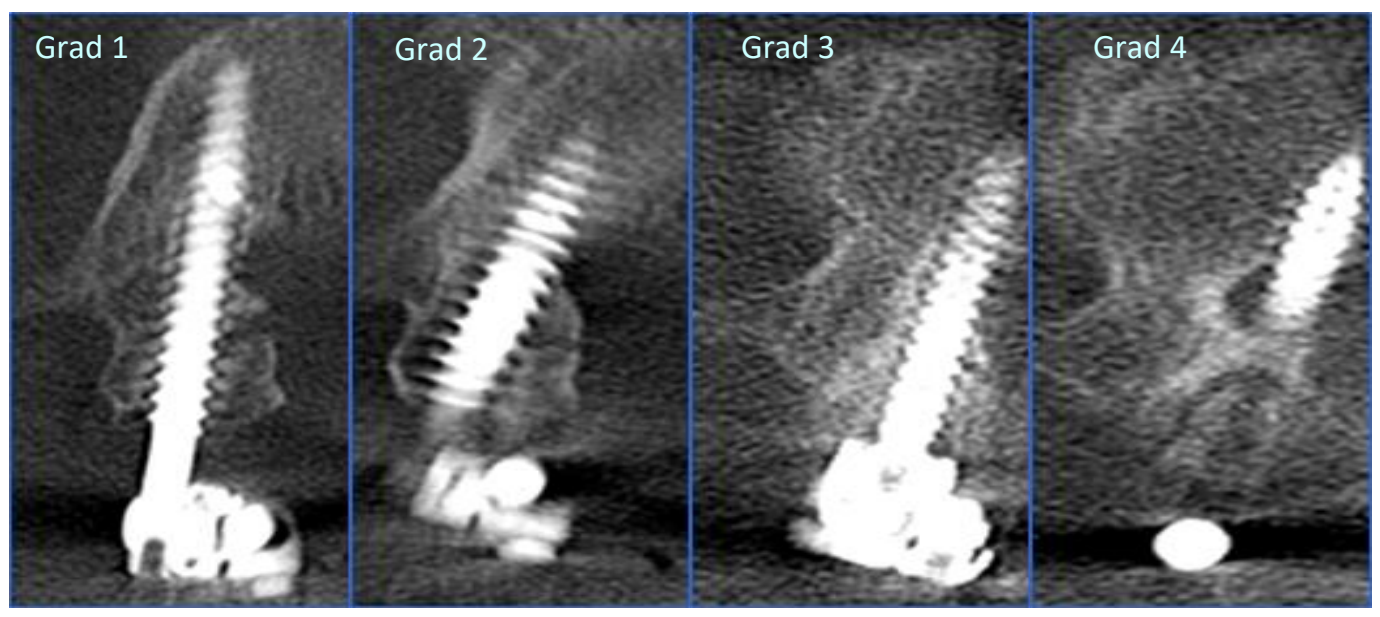

Abbildung 6: Grad 1-4 der Pedikelschraubenfehllage (Kantelhardt et al. 2011, S. 863; die Verwendung erfolgt mit freundlicher Genehmigung der Autoren) 


\subsubsection{Messung des Ho-Winkels}

Der Ho- Winkel ist ein Maß für die Rotation eines Wirbels um die Vertikale. Im Gegensatz zum Cobb-Winkel, der die Krümmung mehrerer Wirbel zueinander misst, wird der Ho-Winkel zur Messung eines einzigen Wirbels genutzt.

Für die Messung wird der zu untersuchende Wirbel in der Computertomographie in der Horizontalebene dargestellt (Abbildung 7). Vor der Winkelmessung wird eine horizontale Linie mit der entsprechenden Vertikale gezogen und drei Anhaltspunkte bestimmt. Der erste Punkt markiert die Verbindungsstelle zwischen den Innenflächen der Laminae arcus vertebrae (Punkt A). Die beiden anderen Punkte werden beidseitig an der Innenfläche der Verbindungsstelle von Lamina und Pediculus arcus vertebrae gesetzt (Punkt $\mathrm{C}$ beidseits). Wenn in der Ansicht ein Pedikel nicht ausreichend dargestellt wird, werden beide Punkte auf der Innenseite der Lamina arcus vertebrae gesetzt, wobei der Abstand zur Verbindungsstelle so gering wie möglich sein sollte. Die Entfernung zur Verbindungsstelle sollte dann jedoch beidseits immer identisch sein. Beide Punkte $\mathrm{C}$ werden über eine Linie mit Punkt A verbunden. Der dadurch entstehende Winkel wird durch eine weitere Linie, die durch den Punkt A geht und den Winkel in der Mitte teilt, gezogen (Linie AX). Die Winkelmessung erfolgt nun zwischen der Linie AX und der Vertikalen (Abbildung 7).

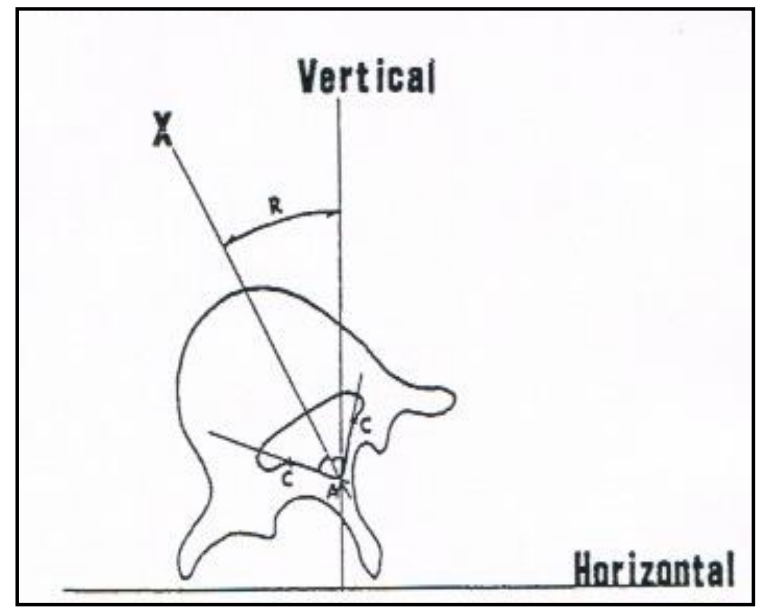

\section{Abbildung 7: Messung des Ho-Winkels (Ho et al. 1993, S 1174; die Verwendung erfolgt mit freundlicher Genehmigung der Autoren)}

Die Messung des Ho-Winkels erfolgte nach ausführlicher Einarbeitung durch die Autorin. Die präoperativen CT-Bilder wurden für die Messung des Ho-Winkels aller zu instrumentierenden Wirbel herangezogen. Aus den gemessenen Winkeln wurde bei jedem Patienten der größte und kleinste Wert in die Datensammlung aufgenommen. Für 
die statistische Auswertung der Daten wurde der maximale Ho-Winkel sowie die Differenz des größten und kleinsten Ho-Winkels bei jedem Patienten genutzt.

Für die Messung der axialen Wirbelrotation stehen eine Reihe weiterer Messtechniken zur Verfügung, wie beispielsweise die Messung nach Nash-Moe, Stokes oder AaroDahlborn, die jedoch für die vorliegende Studie abgelehnt wurden (Aaro et al. 1978; Nash und Moe 1969; Stokes et al. 1986). Die Methode nach Nash-Moe hat zwar den Vorteil, dass intravertebrale Deformitäten keinen Einfluss auf die Genaugikeit der Messung nehmen. Allerdings ist sie ebenso wie die Messung nach Stokes im Vergleich zu anderen Messmethoden weniger akkurat (Russell et al. 1990). Die Messung nach Aaro-Dahlborn und nach Ho sind in ihrer Genauigkeit ebenbürtig. Allerdings konnte gezeigt werden, dass die Messung nach Aaro-Dahlborn in der Anwendung diffiziler und somit ein größeres Potential für Fehlmessungen bietet (Göçen et al. 1998). Daher wurde in der vorliegenden Arbeit die Rotationsmessung nach Ho angewendet.

\subsubsection{Messung der Knochendichte in Hounsfieldeinheiten}

Die Hounsfieldeinheit ist ein Maß für die Absorption von Röntgenstrahlen eines Gewebes in der Computertomographie. Je dichter ein Gewebe ist, desto mehr Röntgenstrahlen werden von diesem absorbiert. Der Wert 0 ist dabei willkürlich der Dichte von Wasser zugeordnet und der Wert -1000 der Dichte von Luft. Daraus folgt, dass je größer die Dichte eines Gewebes ist, desto höher die gemessene HE ist.

Eine erniedrigte Knochendichte birgt das Risiko einer geringeren Stabilität von Implantaten im Knochen (Chen et al. 2011; Coe et al. 1990; Cook et al. 2001). Daher könnte eine Schraubenfehllage mit osteoporöser Knochenmasse in Zusammenhang stehen.

Die akkurateste Methode zur Messung der Knochendichte ist die DEXA (dual-energy $x$ ray absorptiometry) (Mazess et al. 1992). Dabei wird eine Ganzkörperaufnahme mit zwei energetisch unterschiedlichen Röntgenstrahlen gemacht und die unterschiedliche Absorbtion der Gewebe gemessen. Aus dieser lassen sich Fett- und Knochenmasse sowie fettfreie Masse berechnen und zu einem Bild projizieren (Pandey et al. 2011).

Aufgrund des retrospektiven Charakters dieser Arbeit wurde die Messung der HE zur Knochendichteeinschätzung herangezogen. Diese Messung ist zwar nicht Methode der Wahl, dennoch ist sie im klinischen Alltag eine validierte Methode zur Einschätzung 
der Knochendichte (Nilsson et al. 1988; Norton und Gamble 2001; Schreiber et al. 2011; Shapurian et al. 2006).

Zur Bestimmung der Knochendichte wurden die präoperativen CT-Bilder herangezogen und die Hounsfieldeinheit in allen zu verschraubenden Wirbelkörpern gemessen. Anschließend wurde der Mittelwert der HE pro Patient im zu operierenden Wirbelsäulenabschnitt berechnet.

Es erfolgte eine Kategorisierung der Patienten in die Gruppen durchschnittliche HE $<100$ und durchschnittliche HE $\geq 100$. Die Messung erfolgte, nach ausführlicher Einarbeitung durch einem Neuroradiologen, durch die Autorin.

\subsubsection{Messung des Cobb-Winkels}

Die Messung des Cobb-Winkels ist in der Radiologie die am häufigsten angewendete Methode um das Ausmaß einer Skoliose zu erfassen. Der Cobb-Winkel spiegelt die Wirbelsäulenkrümmung in der Frontalebene wider. Dabei werden die Deck- und Grundplatte der beiden Wirbel herangezogen, die am stärksten zueinander verkippt sind. Sie werden Neutralwirbel genannt. Bei der Messung wird eine Linie entlang der Deck- und Grundplatte dieser beiden Wirbel gezogen bis sich die Verlängerungslinien schneiden. Der dadurch entstandene Komplementärwinkel (Cobb-Winkel), dargestellt in Abbildung 8, kann dadurch gemessen werden (Cobb 1947). Der Cobb-Winkel fließt zusammen mit der Klinik des Patienten, dem Alter und der Art der Skoliose in die Entscheidung der zu wählenden Therapie der Skoliose ein.

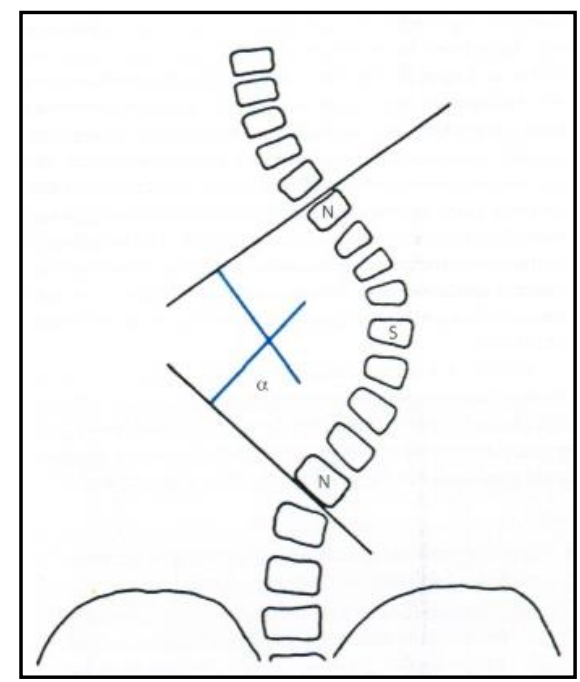

Abbildung 8: Messung des Cobb-Winkels (Linhardt et al. 2011; die Verwendung erfolgt mit freundlicher Genehmigung der Autoren) 
Eine weitere Messmethode für den Krümmungwinkel ist die Messung nach Ferguson. Dabei werden die am stärksten gekrümmten Wirbelkörper als Endwirbel genommen. Als Scheitelwirbel dient der Wirbel, der am wenigsten gekrümmt ist, jedoch die größte Deformität bewirkt. Zur Ermittlung des Mittelpunkts des Scheitelwirbels werden Linien zwischen den halbierten Grund- und Seitenplattenlängen gezeichnet. Der Mittelpunkt der Endwirbel ergibt sich als Schnittpunkt der Diagonalen durch die jeweiligen Endwirbelkörper. Von den Mittelpunkten der Endwirbel wird jeweils eine Verbindungslinie gezogen, die durch den Mittelpunkt des Scheitelwirbels geht. Der so entstandene Komplementärwinkel entspricht dem Skoliosewinkel nach Ferguson (Ferguson 1930). Die Messung nach Ferguson ist aufwendiger als die Skoliosemessung nach Cobb und wird daher im klinischen Alltag seltener genutzt. Daher wurde auch für die vorliegende Arbeit die Messung des Skoliosewinkel nach Cobb vorgenommen.

Das Vorhandensein einer Skoliose wurde in den Patientenakten recherchiert. Sofern eine Skoliose bei einem Patienten vorlag, wurde der Cobb-Winkel in einer präoperativen Röntgen-Aufnahme über den Bereich des operierten Wirbelsäulenabschnitts gemessen. Die Messung erfolgte durch einen erfahrenen Neuroradiologen.

\subsection{Spondylolisthesis}

Die Spondylolisthesis ist die Verschiebung eines Wirbelkörpers und des darüber liegenden Wirbelsäulenabschnitts gegenüber dem darunterliegenden Wirbelkörper. Ätiologisch können sowohl angeborene als auch erworbene Dispositionen zu einer Spondylolisthesis führen. Neben einer Dysplasie des Wirbelbogens oder der Wirbelgelenke sind degenerative und tumoröse Veränderungen sowie Traumata für das Abgleiten der Wirbel verantwortlich. Iatrogen kann eine Spondylolisthesis durch eine Laminektomie provoziert werden, da nach Resektion der dorsalen Anteile des Wirbels eine Instabilität desselben begünstigt wird.

In der Regel geht der Spondylisthesis eine Spondylolyse voraus, welche sich meist im Wirbelbogen in der Pars interarticularis befindet (Spondylolisthesis vera). Durch wiederholte forcierte Extensionen kommt es zu repetitiven Mikrotraumata. Im Verlauf kann dies zur Frakturierung der Pars interarticularis führen. Angeborene Fehlbildungen des Wirbelbogens unterstützen diesen Prozess. Aufgrund des meist beidseitigen Geschehens entsteht eine Hypermobilität des Wirbelkörpers gegenüber dem 
darunterliegenden Wirbelkörper. Durch eine zunehmende Bandscheibendegeneration kann der Prozess noch beschleunigt werden. Schließlich kommt es zum Abgleiten des Wirbelkörpers und der Procc. articulares superiores nach ventral. Der sich dorsal der Lyse vorhandene Rest des Wirbels, bestehend aus unterem Gelenkforsatzpaar, dorsaler Anteil des Arcus vertebrae, Proc. spinosus und Procc. transversi, bleibt an seiner Ursprungsposition zurück. Besondere Risikogruppen sind Patienten mit Hyperlordosebelastung, wie beispielsweise Bergarbeiter oder auch Leistungssportler (Speerwerfen, Kunstturnen), da das Risiko für die Enstehung einer Spondylolyse bei ihnen größer ist als in der Durchschnitssbevölkerung. Die Defektbildungen, die zur Spondyolisthesis führen, befinden sich am häufigsten in LWK5 (80 \%) und LWK4 $(15 \%)$. Hypertrophes Narbengewebe im Bereich der Lyse kann zudem Wurzelreizungen und -kompressionen verursachen.

Degenerativ kann es ebenfalls zur Gefügelockerung mit folgendem Abgleiten des Wirbels kommen. Hier sind keine Defekte der Wirbelbögen ursächlich für das Wirbelgleiten, sondern degenerative Veränderungen der Bandscheibe, weshalb diese Form auch als Pseudospondylolisthesis oder degenerative Spondylolisthesis bezeichnet wird. Sie führt zur Höhenminderung des Intervertebralraums und ventralem Abgleiten des kranialen gegenüber dem kaudalen Wirbel.

Die Patienten entwickeln aufgrund der Instabilität belastungs- und lageabhängige Rückenschmerzen, welche insbesondere beim Stehen auftreten. Eine isthmische Spondylolisthesis kann zu Wurzelreizsymptomatiken und neurologischen Defizite führen (Friberg 1987; Haun und Kettner 2005; Rüther und Lohmann 2014).

Eine Einteilung der Spondylolisthesis erfolgt im sagittalen Röntgenbild nach Meyerding (MD) in fünf Grade (Meyerding 1956; Meyerding 1932). Sie beschreibt den Versatz der Wirbelkörper zueinander in Prozent:

MD I: $\quad$ Versatz der Wirbelkörper zueinander $<25 \%$ der Wirbelkörpertiefe

MD II': Versatz der Wirbelkörper zueinander 25- 50 \% der Wirbelkörpertiefe

MD III ${ }^{\circ}$ : Versatz der Wirbelkörper zueinander 51- $75 \%$ der Wirbelkörpertiefe

MD IV ${ }^{\circ}$ Versatz der Wirbelkörper zueinander $>75 \%$ der Wirbelkörpertiefe MD V': Spondyloptose; kein Kontakt der Wirbelkörper zueinander, oberer Wirbel gleitet nach vorneunten 
Die Patientenakten wurden auf das Vorkommen einer (Pseudo-)Spondylolisthesis im Operationsgebiet untersucht. Bei Vorhandensein wurde die beschriebene Gradeinteilung nach Meyerding mit in die Datenerhebung aufgenommen.

\subsection{Klassifikation des Body-Mass-Index}

Der Body-Mass-Index (BMI) nach Adolphe Quetelet ist ein im klinischen Alltag häufig genutztes Maß zur Einschätzung des Körperfettanteils (Gallagher et al. 1996; Shah und Braverman 2012). Die Berechnung ist simpel und daher einfach anzuwenden. Der BMI ergibt sich aus der Formel:

\section{Körpergewicht $(\mathrm{kg})$}

Body-Mass-Index (BMI) =

$$
\text { Quadrat der Körpergröße }\left(\mathrm{m}^{2}\right)
$$

Die Daten zu Körpergröße und Körpergewicht der Patienten zum Zeitpunkt der Operation wurden aus den Anästhesieprotokollen und Patientenakten erhoben. Der BMI wurde anhand dieser Daten berechnet.

Entsprechend der Klassifikation der World Health Organization (WHO) lässt sich eine Einteilung in Untergewicht, Normalgewicht, Übergewicht und Adipositas vornehmen:

BMI $<18,5 \mathrm{~kg} / \mathrm{m}^{2}$ : Untergewicht

BMI 18,5- 24,9 kg/m²: Normalgewicht

BMI 25- 29,9 kg/m²: Übergewicht

BMI $\geq 30 \mathrm{~kg} / \mathrm{m}^{2}$ : Adipositas

Es erfolgte eine Kategorisierung der Patienten entsprechend der Grenze zur Adipositas in die Gruppen BMI $<30 \mathrm{~kg} / \mathrm{m}^{2}$ und $\geq 30 \mathrm{~kg} / \mathrm{m}^{2}$.

Der BMI gilt für die Einschätzung des Körperfettanteils. Für die Berechnung werden jedoch nur Körpergröße und -gewicht herangezogen. Für eine präzisere Messung des Körperfettanteils steht beispielsweise die DEXA zur Verfügung (Curtin et al. 1997; Pandey et al. 2011; Svendsen et al. 1993).

$\mathrm{Da}$ in vorangegangenen Studien nachgewiesen werden konnte, dass der BMI ein Risikofaktor für Pedikelschraubenfehllage bei konventioneller Technik darstellt, wurde dieser Parameter herangezogen, um einen Zusammenhang der Fehllage auch bei roboterassistierter Spondylodese zu untersuchen (Kim et al. 2011; Yoshii et al.,2015). 


\subsection{Anzahl fusionierter Wirbel}

Das Ausmaß der postoperativen Wirbelsäulenversteifung ist mit der Länge der Spondylodese verbunden. Je mehr Wirbel verschraubt werden, desto höher könnte das Risiko einer Fehllage sein. Daher wurde die Anzahl fusionierter Wirbel durch die Spondylodese in den Operationsberichten und Patientenakten recherchiert.

\subsection{Komplikationen und Reoperation}

Laut Gertzbein und Robbins (1990) befindet sich eine Pedikelschraube mit einer Abweichung $<4 \mathrm{~mm}$ von der vollständigen intrapedikulären Lage in der sicheren Zone. Bei Patienten, die in der oben genannten Pedikelschraubenanalyse mindestens eine Schraube mit einer Fehllage Grad II $^{\circ}$ aufwiesen, wurde die Patientenakte nach postoperativen neurologischen Defiziten untersucht. Sofern diese Komplikationen auftraten, wurde zusätzlich überprüft, ob eine Reoperation des betroffenen Wirbelsäulenabschnitts stattfand.

\subsection{Statistische Auswertung}

Insgesamt wurden die Daten von 211 Patienten untersucht. Die Datensammlung war bei 29 Patienten aufgrund fehlender Informationen unvollständig, weshalb sie für die statistische Auswertung vom Patientenkollektiv ausgeschlossen wurden. Die statistische Auswertung erfolgte mit den Daten von 182 Patienten.

Die elektronische Verarbeitung und Speicherung der Daten erfolgte durch Microsoft Excel ${ }^{\circledR}$ (Microsoft Corporation, 2010). Die statistische Analyse wurde mit dem Programm SPSS Statistics ${ }^{\circledR}$ Version 20 (IBM, USA) durchgeführt. Zur Berechnung der Signifikanzen von Häufigkeitszahlen wurde der Chi-Quadrat-Test sowie der exakte Fisher-Test angewendet. Weiterhin wurden die binäre logistische Regression sowie der Mann-Whitney-U-Test angewendet. Das Signifikanzniveau wurde auf $\mathrm{p}<0,05$ festgelegt. 


\section{Ergebnisse}

\subsection{Verteilung der Pedikelschrauben}

Diese Studie beschreibt 182 konsekutive Patienten, die am Universitätsklinium Göttingen eine perkutane, roboterassistierte Spondylodese erhielten.

Insgesamt wurden 910 Schrauben zwischen BWK2 und SWK2 (Brust- bzw. Sakralwirbelkörper) verschraubt (Tabelle 1). Die Anzahl implantierter Schrauben pro Patient variierte zwischen zwei und 20 Pedikelschrauben, wobei der Median 4 betrug. Die durchschnittliche Anzahl implantierter Schrauben pro Patient betrug fünf Schrauben. Bei den Patienten, die nur zwei Pedikelschrauben erhielten, handelte es sich um eine Anschluss-Spondylodese; nach bereits stattgehabter Spondylodese wurde hierbei ein weiterer Wirbelkörper ober- oder unterhalb an die vorhandene Spondylodese angeschlossen.

Tabelle 1: Anzahl implantierter Schrauben pro Patient

\begin{tabular}{|c||c|c|}
\hline $\begin{array}{c}\text { Anzahl implantierter } \\
\text { Schrauben }\end{array}$ & $\begin{array}{c}\text { Anzahl } \\
\text { Patienten }\end{array}$ & $\begin{array}{c}\text { Prozent } \\
(\%)\end{array}$ \\
\hline \hline 2 & 3 & 1,6 \\
\hline \hline 4 & 127 & 69,8 \\
\hline \hline 5 & 2 & 1,1 \\
\hline \hline 6 & 19 & 12,6 \\
\hline \hline 10 & 4 & 2,2 \\
\hline \hline 12 & 3 & 1,6 \\
\hline \hline 20 & 1 & 5 \\
\hline \hline
\end{tabular}

Mehr als die Hälfte aller Schrauben $(67,4 \%)$ wurde in der Lendenwirbelsäule verschraubt: 613 Schrauben wurden hier implantiert. In der Brustwirbelsäule waren es 
183 Schrauben, in den ersten beiden Sakralwirbeln wurden 114 verschraubt, wobei hiervon nur zwei Schrauben in SWK2 positioniert wurden (Abbildung 9).

Der am häufigsten verschraubte Wirbel war LWK5, insgesamt wurden 200 der 910 Schrauben in diesen Wirbel platziert. Für die Spondylodese wurden in jeweils beide Pedikel eines Wirbels eine Schraube implantiert. Ledigich in zwei Fällen wurde nur eine Schraube in einen Wirbel verschraubt, hierbei war eine beidseitige Bohrung in die Pedikel aufgrund von Instabilität des Wirbels selbst nicht möglich. In diesen beiden Fällen war jeweils nur ein Wirbel innerhalb des zu verschraubenden Segmentabschnitts davon betroffen, die übrigen Wirbel erhielten jeweils zwei Pedikelschrauben.

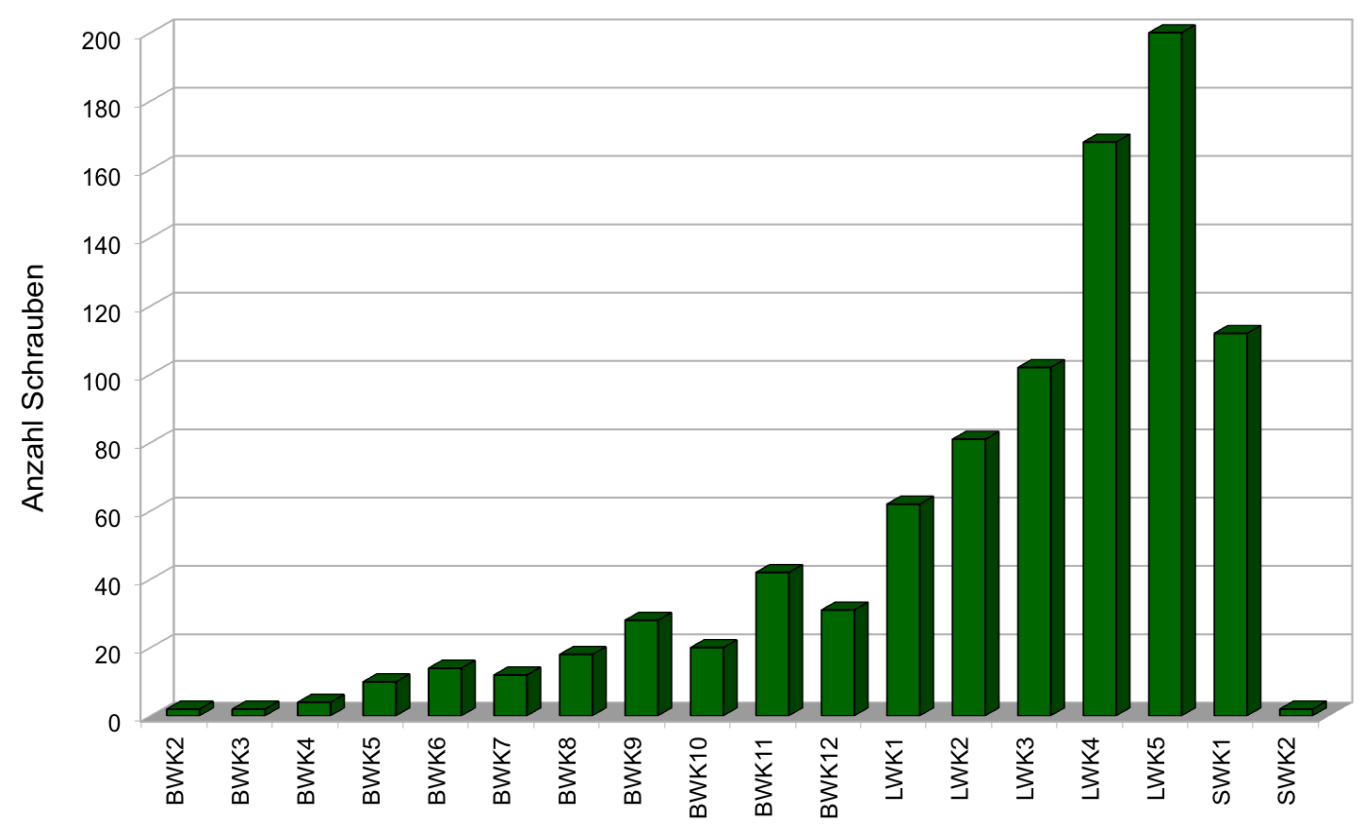

\section{Abbildung 9: Verteilung der Schrauben auf Wirbelkörper}

\subsection{Verteilung korrekter und fehlplatzierter Pedikelschrauben}

Die postoperative Analyse der Schraubenposition im Pedikel ergab bei 117 Patienten vollständig fehlerfrei platzierte Pedikelschrauben.

65 Patienten wiesen Schraubenfehllagen auf, wobei 46 dieser Patienten nur eine einzige Schraubenfehllage hatten. Die maximale Anzahl fehlplatzierter Schrauben pro Patient betrug 4 und betraf zwei Patienten. Bei einem dieser Patienten lagen vier von acht Schrauben (nur Grad I), beim anderen vier von zwölf Schrauben fehl (nur Grad II). 
Sofern Fehllagen bei einem Patienten vorlagen, wurde mit zwei Ausnahmen immer nur ein Grad I oder II festgestellt. Zwei Patienten hatten sowohl eine Schraubenfehllage Grad I als auch eine Schraubenfehllage Grad II.

Die Gesamtzahl korrekt platzierter Schrauben betrug 822 Schrauben (90,3\%). Fehllagen wurden bei 88 Schrauben $(9,7 \%)$ festgestellt, wobei 53 Schrauben einen Grad I und 35 Schrauben Grad II aufwiesen. Im gesamten Kollektiv wurde keine Fehllage Grad III oder IV festgestellt.

Den größten Anteil an Fehllagen bezogen auf die Wirbelkörperhöhe hatte LWK4 mit 16 fehlplatzierten Schrauben (18,2\%). Insgesamt lagen 60 Schrauben in der LWS, 21 Schrauben in der Brustwirbelsäule (BWS) und sieben Schrauben im Os sacrum fehl. Dabei ist jedoch zu berücksichtigen, dass die Gesamtzahl implantierter Schrauben in der LWS ebenfalls am höchsten war. Der prozentuale Anteil von Fehllagen bezogen auf alle implantierten Schrauben im jeweiligen Wirbelsäulenabschnitt betrug 9,8 \% (60/613) für die LWS, 11,5 \% (21/183) für die BWS und 6,1 \% (7/114) für die Sakralwirbelsäule (Abbildung 10; Tabelle 2).

Tabelle 2: Verteilung der Anzahl an Schraubenfehllagen pro Patient auf Anzahl implantierter Schrauben absolut

\begin{tabular}{|l|l|l|}
\hline Fehllagen pro Patient & $\begin{array}{l}\text { Anzahl } \\
\text { Patienten }\end{array}$ & $\begin{array}{l}\text { Anzahl } \\
\text { implantierter } \\
\text { Schrauben }\end{array}$ \\
\hline 0 & 117 & 531 \\
\hline 1 & 46 & 243 \\
\hline 2 & 15 & 96 \\
\hline 3 & 2 & 20 \\
\hline 4 & 2 & 20 \\
\hline
\end{tabular}




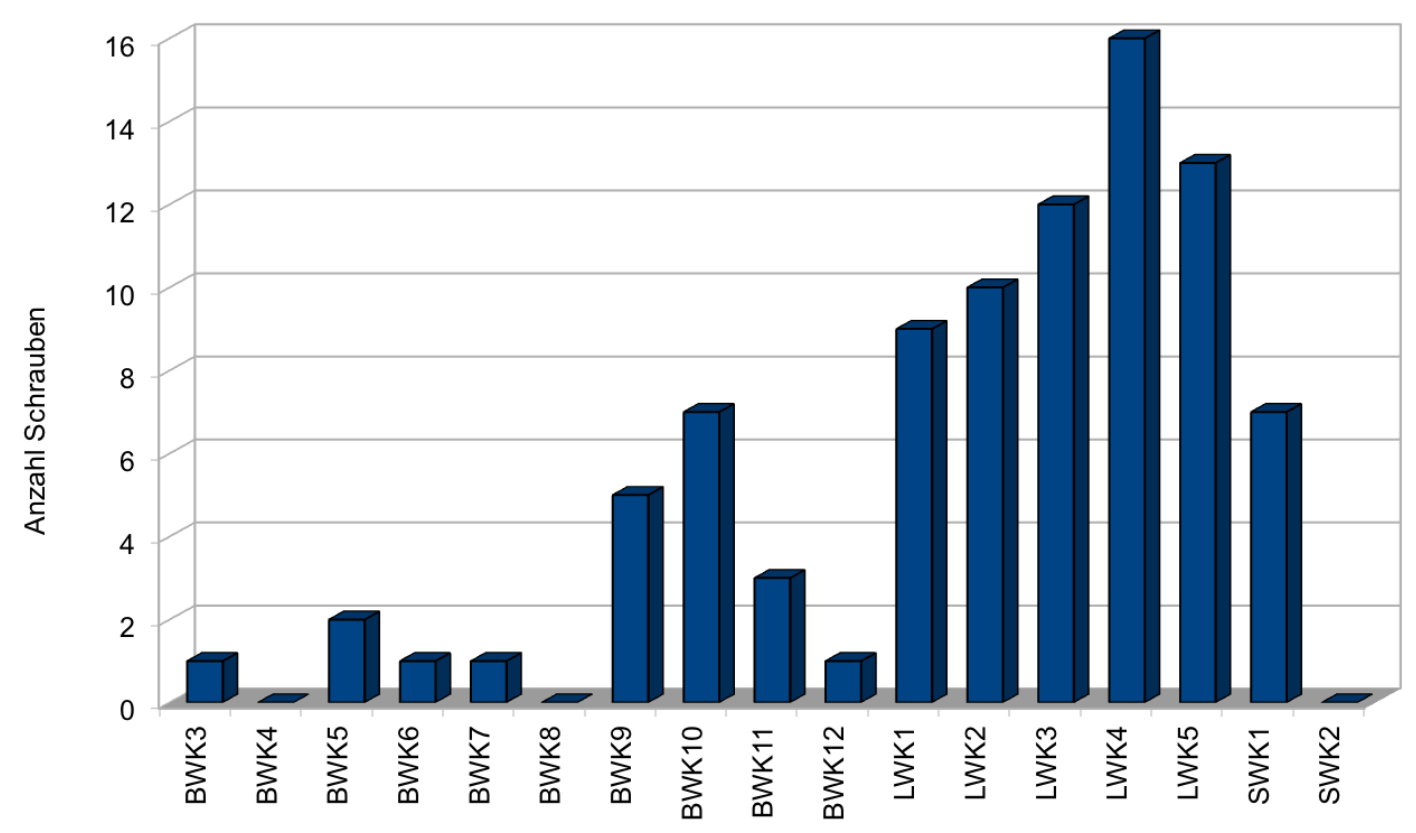

Abbildung 10: Verteilung der Schrauben mit Fehllage auf Wirbelkörper

\subsection{Alters- und Geschlechtsverteilung}

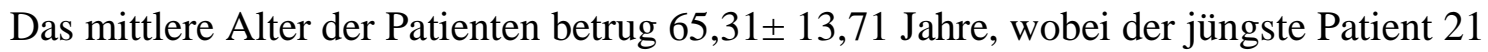
Jahre und der älteste Patient 92 Jahre alt war. Der Altersmedian bei fehlplatzierten Schrauben betrug 69,5 Jahre, bei korrekter Schraubenlage 70 Jahre.

Das Patientenkollektiv umfasste 95 männliche $(52,2 \%)$ und 87 weibliche Patienten $(47,8 \%)$.

Von den 910 implantierten Schrauben wurden 470 bei Männern (51,6\%) und 440 Schrauben bei Frauen (48,4 \%) verschraubt. In der männliche Patientengruppe wiesen 40 Schrauben postoperativ eine Fehllage auf, bei den Frauen lagen 48 Schrauben fehl (Abbildung 11). Es konnte keine statistische Signifikanz zwischen dem Geschlecht und dem Auftreten von Fehllagen gefunden werden $(\mathrm{p}=0,262)$. Weiterhin bestand kein signifikanter Zusammenhang zwischen dem Alter und dem Auftreten von Fehllagen $(p=0,298)$. 


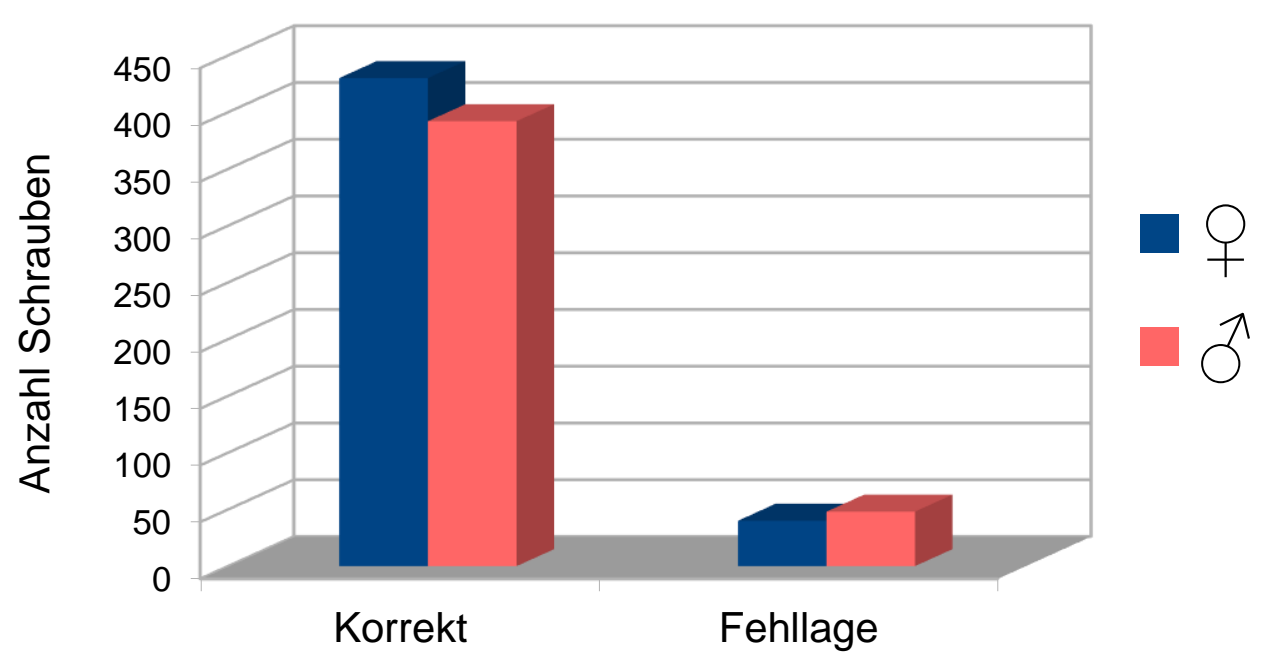

\section{Abbildung 11: Verteilung korrekter und fehlplatzierter Schrauben bei männlichen und weiblichen Patienten}

\subsection{Body-Mass-Index}

Der durchschnittliche BMI des Patientenkollektivs betrug $27,36 \mathrm{~kg} / \mathrm{m}^{2}$, mit einem Median von $26,5 \mathrm{~kg} / \mathrm{m}^{2}$. Der niedrigste berechnete BMI betrug $18 \mathrm{~kg} / \mathrm{m}^{2}$, der größte BMI $46 \mathrm{~kg} / \mathrm{m}^{2}$. 130 Patienten hatten nach der BMI-Klassifikation der WHO ein Normal- oder Übergewicht, 51 Patienten waren adipös und 1 Patient war untergewichtig (BMI: 17,9 kg/m²). Die statistische Analyse des BMIs erfolgte kategorisch mit dem Grenzwert von BMI $<30 \mathrm{~kg} / \mathrm{m}^{2}$ oder $\geq 30 \mathrm{~kg} / \mathrm{m}^{2}$, da dies der Grenzwert für Adipositas ist. Bei 127 Patienten (69,8\%) lag der BMI unter $30 \mathrm{~kg} / \mathrm{m}^{2}, 55$ Patienten $(30,2 \%)$ waren laut BMI adipös.

632 Schrauben wurden bei Patienten verschraubt, die nach dem BMI keine Adipositas aufwiesen. Davon lagen 65 Schrauben (10,3\%) fehl. Bei Patienten mit einem BMI $\geq 30 \mathrm{~kg} / \mathrm{m}^{2}$ wurden 278 Schrauben implantiert, hiervon wiesen 23 (8,3\%) eine Fehllage auf (Abbildung 12). Der mediane BMI war sowohl bei fehlplatzierten als auch bei korrekt positionierten Schrauben $27 \mathrm{~kg} / \mathrm{m}^{2}$. Insgesamt waren $26,1 \%$ aller fehlliegenden Schrauben bei adipösen Patienten verschraubt worden. Die beiden Gruppen konnten keinen signifikanten Unterschied im Bezug zur Fehllage zeigen $(p=0,395)$. 


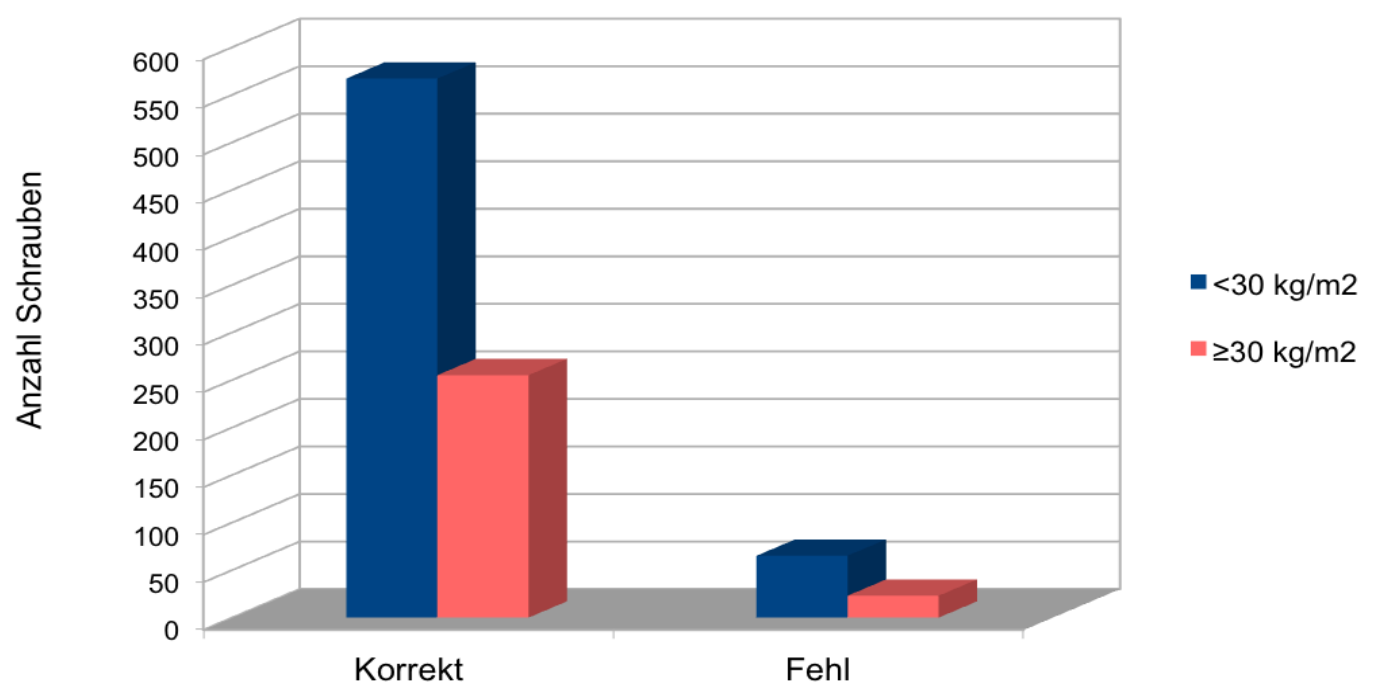

Abbildung 12: Verteilung korrekter und fehlplatzierter Schrauben bei BMI $<$ und $\geq 30 \mathrm{~kg} / \mathrm{m}^{2}$

\subsection{Maximaler Ho-Winkel}

Die Spannbreite des maximal gemessenen Ho-Winkels pro Patient reichte von $0^{\circ}$ bis $25^{\circ}$. Der Median aller implantierten Schrauben betrug $4^{\circ}$, (25./75. Perzentile: $\left.2^{\circ} / 6^{\circ}\right)$. Der mediane maximale Ho-Winkel bei korrekt platzierten Schrauben betrug 3,5 (25./75. Perzentile: $2^{\circ} / 6^{\circ}$ ), der Median bei fehlplatzierten Schrauben betrug $5^{\circ}$ (25/75. Perzentile: $\left.2,25^{\circ} / 8^{\circ}\right)$.

Im Mann-Whitney-U-Test konnte eine statistische Signifikanz festgestellt werden ( $\mathrm{p}=0,001)$. Die logistische Regressionsanalyse war in der univariablen signifikant. Die Odds-Ratio betrug 1,075. Das Risiko einer Fehllage stieg um $7 \%$ pro ansteigendem Grad des Ho-Winkels.

In der mulivariaten Analyse wurde neben dem maximalen Ho-Winkel auch Alter, Geschlecht, BMI, Spondylolisthesegrad, durchschnittliche HE und die Differenz von maximalen und minimalen Ho-Winkel eingeschlossen. Hierbei konnte keine Signifikanz beobachtet werden $(\mathrm{p}=0,666)$. 


\subsection{Differenz von größtem und kleinstem Ho-Winkel}

Um zu erfassen, wie stark die Rotation der Wirbelkörper zur Vertikalen innerhalb des instrumentierten Wirbelsäulenabschnitts war, wurde bei jedem Patienten die Differenz aus größtem und kleinstem Ho-Winkel (Ho-Winkel-Differenz) berechnet. Die kleinste berechnete Differenz betrug $0^{\circ}$, die größte $22^{\circ}$. Die mediane Differenz aller 910 Schrauben betrug $2^{\circ}$.

Die mediane Ho-Winkel-Differenz korrekt platzierter Schrauben betrug $2^{\circ}$ (25./75. Perzentile: $1^{\circ} / 4^{\circ}$ ). Schrauben mit einer Fehllage hatten eine mediane HoWinkel-Differenz von $3^{\circ}\left(25 . / 75\right.$. Perzentile: $\left.2^{\circ} / 6^{\circ}\right)$.

Der p-Wert im Mann-Whitney-U-Test unterschritt das Signifikanzniveau $(p=0,004)$. Ebenso konnte eine statistische Signifikanz in der univariablen Regressionsanalyse festgestellt werden $(\mathrm{p}<0,001)$. Die multivariable Regressionsanalyse ergab keine statistische Signifikanz. Die Odds-Ratio betrug 1,093. Damit stieg das Risiko einer Fehllage um $9 \%$ pro Grad der Ho-Winkel-Differenz.

\subsection{Hounsfieldeinheit}

Der Mittelwert der durchschnittlichen Hounsfieldeinheit pro Patient im vorliegenden Patientenkollektiv war 126,68 HE. Der niedrigste berechnete Wert betrug $38 \mathrm{HE}$, der höchstberechnete Wert war 318 HE. Es erfolgte die Kategorisierung für Patienten mit einer durchschnittlichen HE < und $\geq 100$ HE. 63 Patienten $(34,6 \%)$ wurden in die Gruppe mit einer durchschnittlichen HE $<100$ und 119 Patienten $(64,5 \%)$ in die Gruppe mit einer Durschnitts-HE $\geq 100$ HE eingeteilt.

328 Schrauben wurden bei Patienten mit einer Durschnitts-HE $<100$ verschraubt, davon lagen 38 fehl (11,6\%). Dies waren 43,2 \% aller fehlplatzierten Schrauben. 50 der 582 Schrauben $(8,6 \%)$, die bei Patieten mit einer durchschnittlichen HE von $\geq 100 \mathrm{HE}$ implantiert wurden, wiesen bei der Schraubenpositionsanalyse eine Fehllage auf (Abbildung 13). Dies waren 56,7 \% aller fehlplatzierten Schrauben.

Es konnte keine statistische Signifikanz zwischen einer Fehllage und der durchschnittlichen HE festestellt werden. $(p=0,161)$. 


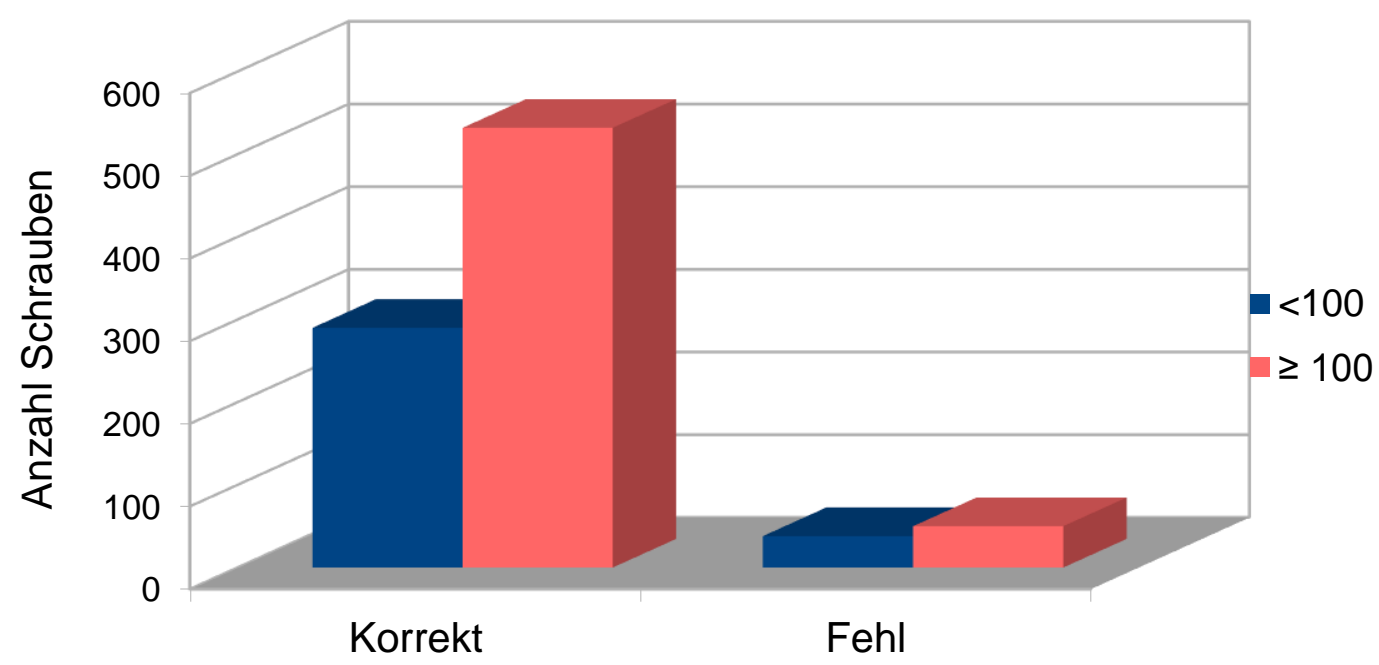

\section{Abbildung 13: Verteilung korrekter und fehlplatzierter Pedikelschrauben auf durchschnittliche Hounsfieldeinheit $<100 \mathrm{HE}$ und $\geq 100 \mathrm{HE}$}

\subsection{Spondylolisthesis}

Im untersuchten Patientenkollektiv konnte keine Spondylolisthesis mit einem Grad > 2 gefunden werden.

133 Patienten hatten kein Wirbelgleiten im Operationsgebiet, 37 Patienten (20,3\%) wiesen eine Spondylolisthesis Grad I und 12 Patienten (6,6\%) einen Grad II nach Meyerding auf.

670 Schrauben wurden bei Patienten ohne Spondylolisthesis implantiert, der Anteil mit Fehllage betrug 8,8 \% (59 Schrauben), wobei dies $67 \%$ aller Schrauben mit Fehllagen waren. 23 von 180 Schrauben (12,8\%), die bei Patienten mit Spondylolisthesis Grad I verschraubt wurden, wiesen eine Fehllage auf, bei Patienten mit einem Grad II waren es sechs von 60 Schrauben (10\%) (Abbildung14).

Der Chi-Quadrat-Test erbrachte keine statistische Signifikanz ( $p=0,277)$. Die bereits in Abschnitt 3.5 erläuterte multivariable Analyse der logistischen Regression ergab jedoch eine Odds-Ratio von 2,178, der p-Wert betrug 0,005. Dies bedeutet ein signifikant erhöhtes Risiko der Schraubenfehllage für Patienten mit einer Spondylolisthesis Grad I. Dieses Risiko ist im Vergleich zu Patienten, die keine Spondylolisthesis haben, um 117 \% erhöht. Für die Spondylolisthesis Grad II bestand sowohl in der univariablen, wie 
auch in der multivariablen logistischen Regressinosanalyse keine statistische Signifikanz ( $\mathrm{p}=0,756$ bzw. $\mathrm{p}=0,562)$.

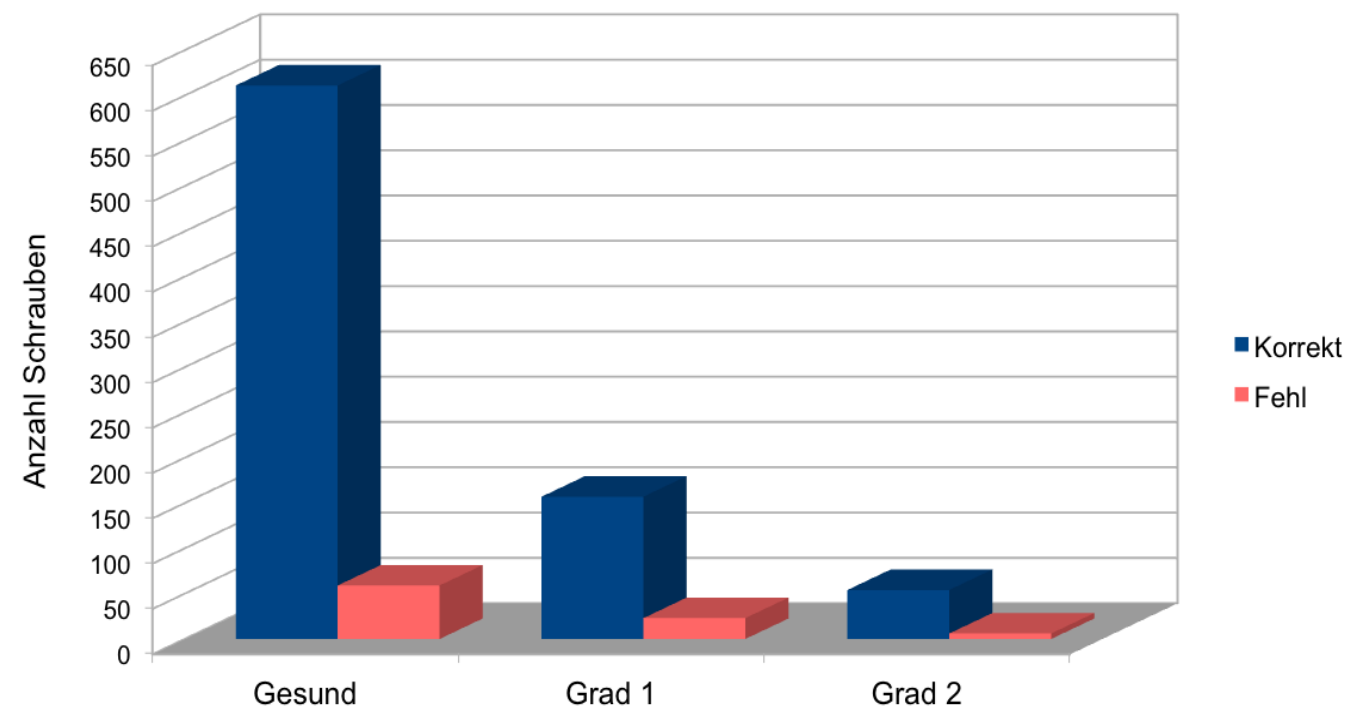

Abbildung 14: Verteilung korrekter und fehlplatzierter Schrauben bei gesunden
Patienten und Patienten mit einer Spondylolisthesis Grad I und II nach Meyerding

\subsection{Cobb-Winkel}

Der Cobb-Winkel wurde bei 47 Patienten bestimmt, da nur diese Patienten im vorhandenen Kollektiv eine Skoliose hatten. Die Anzahl implantierter Schrauben mit vorhandenem Cobb-Winkel betrug daher nur 242 und machte damit einen Anteil von 26,59\% aller verschraubten Pedikelschrauben aus (Tabelle 3). 209 dieser 242 Schrauben wurden korrekt platziert, der Cobb-Winkel betrug hier im Median $15^{\circ}$.

In der Gruppe der fehlplatzierten Schrauben bei Skoliosepatienten wurden 33 Schrauben platziert. Der Median des Cobb-Winkels betrug hierbei $20^{\circ}$.

Der $\mathrm{p}$-Wert lag über dem Signifikanzniveau $(\mathrm{p}=0,066)$, womit zwar kein signifikanter Zusammenhang zwischen Fehllage und Cobb-Winkel festgestellt wurde, jedoch ein gewisser Trend sichtbar wird. Aufgrund der erschwerten Operationsbedingunen und der bereits in Abschnitt 1.7.2 beschriebenen Problematik des SpineAssist ${ }^{\circledR}$ bei starker Deformität der Wirbelsäule die richtige Zielposition anzusteuern, wäre daher ein erhöhtes Fehllagerisiko mit zunehmenden Cobb-Winkel durchaus denkbar. Hierbei ist anzumerken, dass die Anzahl der Schrauben bei Skoliosepatienten im vorliegenden 
Patientenkollektiv sehr gering ist und für eine signifikante Aussage bezüglich Fehllage und Cobb-Winkel ein größeres Kollektiv nötig gewesen wäre.

Tabelle 3: Verteilung des Cobb-Winkels nach korrekter und fehlpositionierter Schraubenlage

\begin{tabular}{|c|c|c|}
\hline & Korrekt & Fehllage \\
\hline Anzahl Schrauben & $209(86,4 \%)$ & $33(13,6 \%)$ \\
\hline Mittelwert & $16,91^{\circ}$ & $22,85^{\circ}$ \\
\hline Standardabweichung & $\pm 11,28^{\circ}$ & $\pm 16,44^{\circ}$ \\
\hline
\end{tabular}

\subsection{Anzahl fusionierter Wirbel}

Die Anzahl fusionierter Wirbel reichte im vorhandenen Patientenkollektiv von eins bis zehn (Tabbelle 4). In drei Fällen wurde eine Anschlussspondylodese durchgeführt, wodurch ein Wirbel zusätzlich versteift wurde. Circa die Häflte aller Patienten $(51,1 \%)$ bekamen zwei Wirbel fusioniert. Der Mittelwert fusionierter Wirbel pro Patient betrug $2,92 \pm 1,424$ Wirbel.

Tabelle 4: Verteilung korrekt und fehlplatzierter Schrauben auf Anzahl fusionierter Wirbel

\begin{tabular}{|l|l|l|l|l|l|}
\hline $\begin{array}{l}\text { Anzahl } \\
\text { fusionierte } \\
\text { Wirbel }\end{array}$ & $\begin{array}{l}\text { Schrauben mit } \\
\text { Fehllage }\end{array}$ & $\begin{array}{l}\text { Anteil } \\
\text { Schrauben mit } \\
\text { Fehllage (\%) }\end{array}$ & $\begin{array}{l}\text { Korrekt } \\
\text { platzierte } \\
\text { Schrauben }\end{array}$ & $\begin{array}{l}\text { Anteil } \\
\text { korrekter } \\
\text { Schrauben }(\%)\end{array}$ & $\begin{array}{l}\text { Gesamtzahl } \\
\text { Schrauben }\end{array}$ \\
\hline 1 & 0 & 0 & 4 & 0,5 & 4 \\
\hline 2 & 25 & 28,4 & 345 & 42 & 370 \\
\hline 3 & 20 & 22,7 & 219 & 26,6 & 239 \\
\hline 4 & 9 & 10,2 & 82 & 10 & 91 \\
\hline 5 & 11 & 12,5 & 71 & 8,6 & 82 \\
\hline 6 & 9 & 10,2 & 45 & 5,5 & 54 \\
\hline 7 & 7 & 8 & 23 & 2,8 & 30 \\
\hline 8 & 3 & 3,4 & 9 & 1,1 & 12 \\
\hline 9 & 3 & 3,4 & 5 & 0,6 & 8 \\
\hline 10 & 1 & 1,1 & 19 & 2,3 & 20 \\
\hline
\end{tabular}


Es bestand eine statistische Signifikanz zwischen der Anzahl fusionierter Wirbel und dem Auftreten einer Fehllage $(p=0,002)$. Hierbei ist jedoch $z u$ vermerken, dass die Anzahl an fehlplatzierten Schrauben, die bei der Fusion von einem, sieben, acht und neun Wirbeln verschraubt wurden, <5 war und die Aussage der Signifikanz dadurch abgeschwächt wird.

Sowohl die uni- als auch die multivariable logistische Regressionsanalyse ergab eine statistische Signifikanz. Für die univariable Analyse betrug der p-Wert $<0,001$, die Odds Ratio betrug 1,201. Die multivariable Analyse ergab eine Odds-Ratio von 1,185, der p-Wert betrug 0,006. Dies bedeutet, dass das Fehllagerisiko pro fusioniertem Wirbel um $20 \%$ in der univariablen und um $18 \%$ in der multivariablen Analyse anstieg. 


\subsection{Tabellarische Zusammenfassung der statistischen Auswertung}

Tabelle 5: Verteilung und Signifikanz von demographischen und winkelassoziierten Parametern sowie Spondylolisthesis und HE.

\begin{tabular}{|c|c|c|c|c|c|}
\hline & & $\begin{array}{l}\text { Schrauben } \\
\text { mit } \\
\text { Fehllage }\end{array}$ & $\begin{array}{l}\text { Korrekte } \\
\text { Schrauben- } \\
\text { lage }\end{array}$ & Total & $\mathrm{p}^{*}$ \\
\hline Alter (Jahre) & Median & $69,5(60-77)$ & $70(57-76)$ & $70(57,75-76)$ & 0,298 \\
\hline \multirow[t]{2}{*}{ Geschlecht } & Männlich & $40(45,5 \%)$ & $430(52,3 \%)$ & 470 & \multirow[b]{2}{*}{0,262} \\
\hline & Weiblich & $48(54,5 \%)$ & $392(47,7 \%)$ & 440 & \\
\hline \multirow[t]{2}{*}{ BMI } & $<30 \mathrm{~kg} / \mathrm{m}^{2}$ & $65(73,9 \%)$ & $567(69 \%)$ & 632 & \multirow[b]{2}{*}{0,395} \\
\hline & $\geq 30 \mathrm{~kg} / \mathrm{m}^{2}$ & $23(26,1 \%)$ & $255(31 \%)$ & 278 & \\
\hline \multirow[t]{3}{*}{ Spondylolisthesis } & Gesund & $59(67 \%)$ & $611(74,3 \%)$ & 670 & \multirow[b]{3}{*}{0,277} \\
\hline & Grad I & $23(26,1 \%)$ & $157(19,1 \%)$ & 180 & \\
\hline & Grad II & $6(6,8 \%)$ & $54(6,6 \%)$ & 60 & \\
\hline \multirow{2}{*}{$\begin{array}{l}\text { Durchschnittliche } \\
\text { HE }\end{array}$} & $<100 \mathrm{HE}$ & $38(43,2 \%)$ & $290(35,3 \%)$ & 328 & \multirow[b]{2}{*}{0,161} \\
\hline & $\geq 100 \mathrm{HE}$ & $50(56,8 \%)$ & $532(64,7 \%)$ & 82 & \\
\hline $\begin{array}{l}\text { Max. Ho-Winkel } \\
(\mathrm{Grad})\end{array}$ & Median & $5(2,25-8)$ & $3,5(2-6)$ & $4(2-6)$ & 0,001 \\
\hline $\begin{array}{l}\text { Differenz max. } \\
\text { und min. Ho- } \\
\text { Winkel (Grad) }\end{array}$ & Median & $3(2-6)$ & $2(1-4)$ & $2(1-4)$ & 0,004 \\
\hline $\begin{array}{l}\text { Cobb-Winkel } \\
(\text { Grad) }\end{array}$ & Median & $20(8-30)$ & $15(9-21)$ & $15(9-22,75)$ & 0,066 \\
\hline
\end{tabular}

* kontinuierliche Daten: Mann-Whitney-U-Test, kategorische Daten: Chi-Quadrat-Test und Exakter Test nach Fisher 
Tabelle 6: Uni- und multivariable logistische Regressionsanalyse

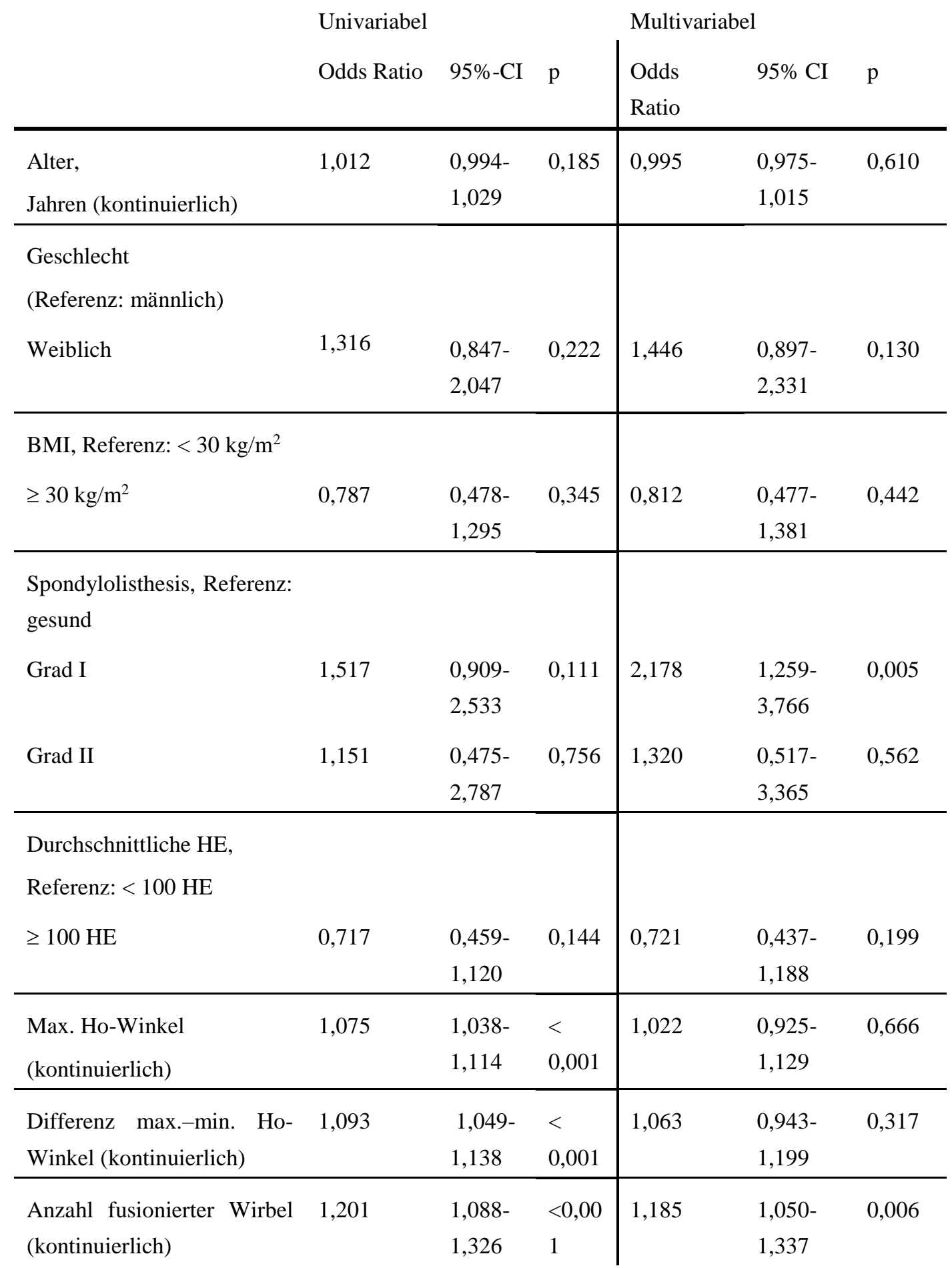




\subsection{Komplikationen und Reoperation}

Postoperative neurologische Defizite bei Schraubenfehllage Grad II traten bei vier Patienten auf.

$\mathrm{Zu}$ den Komplikationen gehörten in einem Fall Paresen der unteren Extremität ohne sensibles Defizit aufgrund einer osteolytischen Spondylodiszitis. Erwähnenswert ist hierbei allerdings, dass die Fehllagen Grad II im lumbosakralen Wirbelsäulenabschnitt lagen, während die Spondylodiszitis den mittleren Brustwirbelsäulenabschnitt betraf. Somit ist die Parese nicht auf die Fehllage der Pedikelschrauben zurückzuführen. Im Fall der aufgetretenen Parese wurde aufgrund der Symptomatik eine Reoperation durchgeführt. 


\section{Diskussion}

Die vorliegende retrospektive Studie hat die Genauigkeit der roboterassistierten perkutanen Pedikelschraubenimplantation in der thorako-lumbo-sakralen Wirbelsäule und mögliche Fehlerquellen bei Schraubenfehllage untersucht. Dafür wurde die postoperative Schraubenposition analysiert (nach CT-Diagnostik) und in ein Grading, welches sich an die international verwendeten Klassifikationen von Wiesner et al. (1999) und Schizas et al. (2007) anlehnt, eingeteilt. Außerdem wurden Daten bezüglich Demographie, Deformität inklusive Rotationswinkel von einzelnen Wirbeln und Wirbelsäulenabschnitten sowie Knochendichte und sagittale Deformitäten gemessen und zur Analyse in die Datensammlung mit aufgenommen.

Die Vorteile des SpineAssist ${ }^{\circledR}$ und seine verbesserte Genauigkeit bei der Pedikelschraubenimplantation konnten in mehreren Studien nachgewiesen werden (Kantelhardt et al. 2011; Pechlivanis et al. 2009; Roser et al. 2013; Togawa et al. 2007). Auch wenn die Fehllageraten auf minimale Fallzahlen reduziert werden konnten, ist eine vollständig einwandfreie Positionierung der Pedikelschrauben auch unter Verwendung des SpineAssist ${ }^{\circledR}$ bisher nicht erreicht worden. Zum jetzigen Zeitpunkt sind der Autorin keine Studien bezüglich der Zusammenhänge roboterassistierter Fehllagen und klinischer oder technischer Parametern bekannt. Ein Vergleich mit anderen Studien, die die Ursachen von Fehllagen bei roboterassistierter Pedikelschraubenimplantation analysiert haben, ist somit nicht möglich. Ziel der Arbeit war es dennoch, die bisherige Datenlage bezüglich der Genauigkeit des Robotersystems zu bestätigen und Zusammenhänge zwischen den gesammelten Parametern und Schraubenfehllagen zu analysieren.

\subsection{Genauigkeit der Schraubenpositionierung durch den SpineAssist ${ }^{\circledR}$}

Die Analyse der postoperativen Schraubenposition ergab eine korrekte Schraubenposition in 90,3\%. Bei 5,8 \% der Schrauben (53 Schrauben) waren Fehllagen Grad I zu beobachten, während 3,9\% (35 Schrauben) Fehllagen Grad II nachwiesen. Eine Revisionsoperation war bei nur drei Patienten nötig und betraf die Position von 
sieben Schrauben. Damit lagen insgesamt 99,2\% der Schrauben nach klinischen Gesichtspunkten korrekt.

Die genannten Fehllageraten sind mit der bisherigen Datenlage bezüglich der Genauigkeit durch den SpineAssist ${ }^{\circledR}$ vergleichbar. Kantelhardt et al. (2011) hatten bei roboterassistierter perkutaner Spondylodese eine Fehllagerate von 6\%. Roser et al. (2013) wiesen bei 62 Patienten eine Fehllage in $8 \%$ nach.

Die vorliegenden Zahlen sind jedoch kritisch zu bewerten, da in den genannten Studien unterschiedliche Klassifikationen für die Schraubenfehllage und Operationstechniken benutzt wurden. Das in dieser Studie genutzte Grading lehnte sich an die Klassifikation nach Wiesner et al. (1999) und Schizas et al. (2007) an. Andere Studien nutzten jedoch komplett davon abweichende Klassifikationen. So teilten beispielsweise Farber et al. (1995) die Schraubenfehllage in in, out oder questionable (innerhalb, außerhalb, fragwürdig) ein, was im Vergleich mit anderen Studien eine ungenaue Einteilung darstellt. Währendessen wurden die Fehllagen von Gertzbein und Robbins (1990) in eine 2-mm-Skalierung eingeteilt. Liljenqvist et al. (1997) maßen in 1-mm-Abständen und klassifzierte Fehllagen nicht weiter. Diese Problematik wurde bereits von Kosmopoulos und Schizas (2007) aufgezeigt. In den von ihnen untersuchten 130 Studien wurden 35 verschiedene Klassifikationssysteme für die Analyse von Pedikelschrauben angewendet. Sie kritisierten, dass der Vergleich von Studien mit unterschiedlichen Klassifikationen zu irreführenden und daher möglicherweise falschen Ergebnissen und Messungen führen könnte. Eine Vergleichbarkeit der unterschiedlichen Studienergebnisse ist somit nur eingeschränkt möglich. Eine international einheitliche Klassifikation könnte diese Problematik lösen, womit zukünftige Studien im Vergleich besser zu deuten wären.

Die in der vorliegenden Arbeit genutzte Klassifikation wäre eine gute Methode, Pedikelschraubenpositionen $\mathrm{zu}$ bewerten: die hier genannten Grad 0 und Grad I befinden sich in der von Gertzbein und Robbins (1990) genannten sicheren Zone von 0$4 \mathrm{~mm}$. In dieser Abweichungszone traten laut ihrer Studie keine neurologischen Komplikationen auf und sie bewerteten diesen Abstand daher als unbedenklich. Mit der Einführung der in dieser Arbeit genutzten Einteilung wäre die Möglichkeit gegeben, in zukünftigen Studien eine exaktere Untersuchung der von ihnen als korrekt klassifizierten Schrauben durchzuführen. Das genannte Grading bietet zudem durch seine vier Schweregrade die Möglichkeit, bereits minimale Abweichungen zu erfassen, wodurch eine gute Übersicht über die vorhandenen Abweichungen geschaffen wird. 
Somit ist eine bessere Einschätzung der $\mathrm{zu}$ erwartenden Komplikationen bei entsprechendem Grading möglich.

\subsection{Alter und Geschlecht}

Die Morphologie des Pedikels wurde in vielen Studien untersucht (Hou et al. 1993; Krag et al. 1988; McCormack et al. 1995; Zindrick et al. 1967). Die Unterschiede zwischen der Anatomie bei männlichen und weiblichen Patienten sind dabei jedoch nur eingeschränkt untersucht. Hou et al. (1993) untersuchten die Morpholgie des Pedikelknochen im thorakolumbalen Wirbelsäulenabschnitt bei einer chinesischen Population und analysierten die Unterschiede bei Männern und Frauen. Hierbei zeigte sich eine signifikant kleinere Pedikelbreite bei chinesischen Frauen in BWK9 und BWK12. Zusätzlich verglichen sie ihre Ergebnisse mit denen von Studien, die die Morphologie eines weißen Patientenkollektivs untersucht hatten. Unter diesen war nur eine Studie, die ebenfalls zwischen männlichen und weiblichen Patienten unterschieden hatte. Die Pedikelbreiten des männlichen Patientenkollektivs von Hou et al (1993) waren dabei signifikant kleiner als die der männlichen weißen Bevölkerung von Olsewski et al. (1990). Olsewksi et al. (1990) hatten zuvor in ihrer eigenen Studie ebenfalls nachweisen können, dass die Breitenwerte der lumbalen Pedikelknochen bei Männern größer sind als bei Frauen.

In der vorliegenden Untersuchung konnte kein signifikanter Zusammenhang zwischen Schraubenfehllage und Geschlecht gefunden werden $(p=0,262)$. Die Verteilung der männlichen und weiblichen Patienten im vorliegenden Kollektiv war anähernd gleich (52,2 \% männliche und 47,8\% weibliche Patienten), sodass die Aussagekraft der statistischen Auswertung damit bekräftigt ist.

Die Pedikelmorphologie selbst ist zwar sehr gut dokumentiert, diverse Studien haben dabei jedoch keinen Unterschied zwischen der Pedikelmorphologie der Geschlechter untersucht. Für die Fixation der Pedikelschrauben scheinen daher nicht die geschlechtlichen Unterschiede der Pedikellängen- und Breiten entscheidend zu sein, sondern ein präzises Auswählen der Schraubenlänge und ihres Durchmessers (Krag et al. 1988; Misenhimer et al. 1989). Dies kann durch die prä- und intraoperative Planung des SpineAssist ${ }^{\circledR}$ gewährleistet werden. 
Morales-Avalos et al. (2014) untersuchten die Veränderungen der Pedikelanatomie bei zunehmendem Alter. Die Auswertung von 4800 Pedikelknochen ergab eine Abnahme des Pedikeldurchmesser bei Frauen mit zunehmendem Alter. Interessanterweise fanden sie einen gegensätzlichen Trend bei Männern, deren Pedikeldurchmesser im thorakalen Wirbelsäulenbereich mit zunehmendem Alter größer werden. Als Ursache dessen vermuteten sie hierfür eine unterschiedliche, multifaktorielle Entwicklung der Knochenstruktur, die durch Ernährung, physische Belastung der Knochen sowie endokrine und osteodegenerative Faktoren beeinflusst werden könnte. Dies steht im Kontrast zu den Ergebnissen von Mughir et al. (2010), die den Durchmesser und die Länge des Pedikelknochens von Jugendlichen und Erwachsenen verglichen und dabei signifikant kleinere Werte bei der (älteren) erwachsenen Subgruppe feststellte und diese unabhängig vom Geschlecht waren.

Die vorliegende Datenanalyse konnte keine Signifikanz zwischen Schraubenfehllagen und Alter nachweisen $(p=0,298)$. Die Morphologie scheint also auch durch degenerative Prozesse, die physiologisch mit zunehmenden Alter auftreten, keinen signifikanten Einfluss auf die Präzision des SpineAssist® zu nehmen.

\subsection{Body-Mass-Index}

Yoshii et al. (2015) wiesen in ihrer Studie nach, dass bei konventioneller Technik fettleibige Patienten mit einem BMI $\geq 25 \mathrm{~kg} / \mathrm{m}^{3}$ ein erhöhtes Risiko für eine Schraubenfehllage haben. Das Risiko einer Fehllage bei Patienten mit einem BMI $\geq 25 \mathrm{~kg} / \mathrm{m}^{3}$ war in dem untersuchten Patientenkollektiv $359 \%$ höher als bei Patienten ohne Übergewicht oder Adipositas. Das erhöhte Risiko für Pedikelschraubenfehllage bei Fettleibigkeit konnte auch durch Kim et al. (2011) bestätigt werden. Dies steht im Kontrast zu den Ergebissen dieser roboterassistierten Studie, die keine Signifikanz zwischen BMI und Fehllage feststellen konnte. Allerdings wurden die hier vorliegenden Berechnungen für Patienten mit Adipositas, die als BMI $\geq 30 \mathrm{~kg} / \mathrm{m}^{3}$ definiert ist, durchgeführt. Eine mögliche Erklärung dafür könnte daher zum einem die unterschiedlich festgesetzten Kategorisierungsgrenzen sein. Zum anderen wurde bei Yohii et al. (2015) eine konventionelle Operationstechnik angewendet, die, wie bereits in Abschnitt 1.3 berichtet, insgesamt auch eine erhöhte Fehllagerate aufweist. Durch den vergrößerten Weichteilmantel liegen die Pedikel weiter von der Oberfläche entfernt 
und die Sicht auf das OP-Gebiet ist limitiert (Yoshii et al. 2015). Aufgrund der unterschiedlichen Operationstechniken ist es daher gut vorstellbar, dass hier Risikofaktoren vorlagen, die bei der roboterassistierten Technik nicht von Relevanz sind.

Die Qualität von Röntgen-Aufnahmen, die mittels Röntgenstrahlen aufgenommen werden, hängt von dem vorhandenen Fettgewebe ab. Da Fettgewebe, wie jedes andere Gewebe auch, Röntgenstrahlen absorbiert, wird die Qualität der Aufnahme umso schlechter, je mehr Fettgewebe vorhanden und je größer dadurch der Weichteilmantel des Patienten ist (Uppot et al. 2007; Uppot 2006). Daher stellte sich die Frage, ob bei zunehmendem BMI die Workstation des SpineAssist ${ }^{\circledR}$ in der Lage ist, trotz Qualitätsminderung der Bilder die korrekten Positionierungsdaten für den Roboter zu berechnen.

Das Ergebnis zwischen Schraubenfehllage und BMI war in der vorliegenden Untersuchung statistisch nicht signifikant $(\mathrm{p}=0,395)$. Das Fehlen einer Signifikanz könnte darauf zurückzuführen sein, dass die Software der Workstation des SpineAssist ${ }^{\circledR}$ in der Lage ist, nach erfolgreicher Registrierung die Qualitätsminderung der Bilder adipöser Patienten zu überwinden. Damit ist ein adäquates Matching durchführbar und der SpineAssist ${ }^{\circledR}$ erhält die korrekten Informationen zur Schraubenplatzierung. Eine beim Patienten vorliegende Adipositas stellt somit keine Einschränkung für eine roboterassistierte Spondylodese dar.

Nichtsdestotrotz werden zur Berechnung des BMIs nur Körpergewicht und -größe des Patienten miteinbezogen. Weitere Faktoren, wie beispielsweise Geschlecht, Alter und Muskelmasse werden dabei nicht berücksichtigt. Dadurch kann der BMI zu Fehleinschätzungen des wahren Körperfettanteils führen (Gallagher et al. 1996; Shah und Braverman 2012; Stenholm et al. 2008). Um die hier aufgestellte These überprüfen zu können, sollte daher der Körperfettanteil mit präziseren Methoden, wie beispielsweise der DEXA (s. Abschnitt 2.5), erneut gemessen und der Zusammenhang zwischen diesen Werten und dem Auftreten von Fehllagen bei roboterassistierter Spondylodese untersucht werden. 


\subsection{Maximaler Ho-Winkel und Differenz von größtem und kleinstem Ho-Winkel}

Bereits Barzilay et al. (2006) und $\mathrm{Hu}$ et al. (2013) hatten aufgezeigt, dass der SpineAssist ${ }^{\circledR}$ bei starker Rotation der Wirbelsäule die Zielposition nicht erreichen konnte und damit eine roboterassistierte Verschraubung nicht möglich war. Die Positionierung der Klammer bzw. des Hover-T-Rahmens bezieht sich auf den ersten zu operierenden Wirbel. Liegt nun eine starke Rotation der anschließend zu verschraubenden Wirbel vor, wie es bei einer großen Differenz von maximalen und minialem Ho-Winkel der Fall ist, sind dies erschwerte Positionierungsbedingungen für den SpineAssist ${ }^{\circledR}$.

In der vorliegenden Untersuchung konnte gezeigt werden, dass das Risiko einer Fehllage pro zunehmender Differenz des maximalen und minimalen Ho-Winkels um $9 \%$ ansteigt. Dies kann auch im Einklang mit dem Ergebnis des maximalen HoWinkels gesehen werden: Mit zunehmendem maximalen Ho-Winkel steigt das Risiko einer Fehllage um $7 \%$ pro Winkelgrad. Zum einem ist also die Rotation eines einzelnen Wirbels zur Vertikalen entscheidend, zum anderen die Rotation der zu operierenden Wirbel untereinander.

Ein Vorteil der bildgebenden Chirurgie ist, dass die Rotation der Wirbel prä- und intraoperativ vollumfängich erfasst werden kann und sich nicht nur auf die äußerlichen Landmarken beschränkt. Da für die Planung der roboterassistierten Spondylodese präoperative CT-Bilder angefertigt werden, könnten diese zusätzlich genutzt werden, eine starke Rotation der Wirbel sowohl selbst als auch zueinander bereits im Vorfeld aufzudecken. Im eintretenden Fall könnte dann während der OP die Position der Klammer oder des Hover-T-Rahmens bei Bedarf neu gesetzt werden, um eine korrekte Schraubenpositionierung zu garantieren. Dadurch könnte die Fehllagerate beim Einsatz des SpineAssist ${ }^{\circledR}$ weiter gesenkt werden.

Die präoperative Analyse der Wirbelrotation ist daher für die Planung der Spondylodese bedeutsam. 


\subsection{Hounsfieldeinheit}

Die Messung der Knochendichte ist im klinischen Alltag von Bedeutung, da sie zur Einschätzung von osteoporösen Vorgängen und Frakturrisiko dient (Celenk und Celenk 2012). Durch eine verminderte Knochendichte ist die Pedikelschraubeninsertion bei Spondylodese erschwert, weshalb Kenntnisse über osteoporotische Vorgänge im Operationsgebiet für das korrekte Einbringen der Pedikelschrauben nicht unerheblich sind.

In der Literatur findet sich eine Anzahl von Studien, die die Hounsfieldeinheit als eine validierte Methode für die Messung der Knochendichte ansehen (Nilsson et al. 1988; Norton und Gamble 2001; Schreiber et al. 2011; Shapurian et al. 2006). Ungenauigkeiten der HE-Messung entstehen jedoch durch einen zunehmden Weichteilmantel: nimmt dieser im gemessenen Volumen zu, so nimmt die HE-Zahl ab (Celenk und Celenk 2012).

Aufgrund der genaueren Messwerte ist der Goldstandard für die Knochendichtemessung daher nicht die HE-Messung, sondern die dual-energy $x$-ray absorptiometry (Mazess et al. 1992). Aufgrund des retrospektiven Charakters dieser Arbeit war eine DEXAMessung jedoch nicht möglich.

Das Einbringen der Pedikelschrauben ist bei Osteoporose-Patienten durch die brüchige Struktur des Knochens erschwert (McAfee et al. 1991; West et al. 1991; Whitecloud et al. 1989). In der vorliegenden Arbeit wurde jedoch kein Zusammenhang zwischen der durchschnittlichen HE und Schraubenfehllage nachgewiesen. Daher scheint eine roboterassistierte Spondylodese auch bei Patienten mit Osteoporse zulässig zu sein. Für die Zuverlässigkeit dieser Aussage sollte in zukünfitgen Studien dennoch der Zusammenhang zwischen Fehllage und Knochendichte mittels DEXA-Messung untersucht werden. Dadurch würden Schwachstellen der HE-Messung ausgeglichen und die Reliabilität dieser Aussage geprüft werden können. 


\subsection{Spondylolisthesis}

Die Datenauswertung der Pedikelschrauben ergab ein erhöhtes Risiko einer Schraubenfehllage bei Spondylolisthesis Grad I gegenüber gesunden Patienten, die Odds Ratio betrug 2,178 ( $\mathrm{p}=0$,005). Für Grad II wurde kein signifikant erhöhtes Risiko gefunden. Dieses Ergebnis erscheint zunächst kontrovers, da man bei schwerwiegenderem Gleiten der Wirbel eher mit Problematiken bei der Schraubeninsertion rechnen würde.

Eine Schwachstelle der vorliegenden Untersuchung ist, dass im vorliegenden Patientenkolletiv 37 Patienten mit Olisthesis Grad I und 12 Patienten mit Olisthesis Grad II vorhanden waren. Insgesamt wurden 180 Schrauben in der ersten genannten Gruppe und 60 Schrauben in der zweiten genannten Gruppe implantiert. Damit ist das Verhältnis dieser beiden Gruppen unausgeglichen, die Reliabilität ist somit leider anzuzweifeln.

Um das vorliegende Ergebnis zu untermauern bzw. zu entkräftigen, wäre daher eine Untersuchung nötig, bei der eine ausreichende Anzahl von Patienten mit Olisthesis Grad II (oder höher) eingebunden sind. Dennoch ist ein erhöhtes Risiko bei Patienten mit Spondylolisthesis Grad I im Vergleich zu Patienten ohne diese Pathologie zu bemerken.

Die Pedikelanatomie bei Patienten mit spondolytischen Spondylolisthesis ist aufgrund der Pathologie verändert und trägt zu einer Verlängerung der Pedikellänge bei (Matthews et al. 2015). Dies in Kombination mit einem Frakturspalt in der Pars interarticularis, wie er bei spondylolytischer Spondylolisthesis vorzufinden ist, kann zu einer vorderen, hinteren und winkelbedingten Instabilität des Wirbels führen (Niggemann et al. 2011). Hierbei kann es also zu einer Makroinstabilität des Wirbels kommen. Die vorliegende Arbeit zeigt ein erhöhtes Fehllage-Risiko bei Patienten mit Spondylolisthesis. Es ist daher gut vorstellbar, dass aufgrund der Makroinstabilität eine definitive Lage der Wirbelbestandteile (und damit auch der Pedikel) während der mechanische Einbringung nicht genau zu definieren ist und daher eine exakte Einbringung trotz genauem Arbeiten des SpineAssist ${ }^{\circledR}$ erschwert sein könnte.

Umso wichtiger ist daher eine prä- und intraoperativ möglichst exakte Bohrplanung und Verschraubung der Pedikelschrauben, damit das Risiko einer Fehlplatzierung weiterhin gering bleibt. 


\subsection{Cobb-Winkel}

Vor der Einführung der roboterassistierten Operationstechnik wurden bereits durch Liljenqvist et. al (1997) erschwerte Operationsbedingungen bei Skoliosepatienten beschrieben. Aufgrund der rotierten Anatomieverhältnisse war eine Orientierung anhand der gegebenen Landmarken zu diesem Zeitpunkt nur eingeschränkt möglich. Dennoch ist die Pedikelschraubentechnik bei Skoliosepatienten Therapie der Wahl, da bei geringerer Fusionslänge eine bessere Korrektur der Deformität möglich ist als mit anderen Operationsmethoden (Halm et al. 2000; Suk et al. 1994; Wimmer et al. 2001). Bei konventioneller Operationstechnik sind Pedikelschraubenfehllageraten bei Patienten mit spinaler Deformität von 23-25\% beschrieben worden (Rajasekaran et al. 2007; Liljenqvist et al. 1997).

In der vorliegende Arbeit wurde untersucht, ob bei roboterassistierter Spondylodese ein Zusammenhang zwischen Fehllagerate und starker Wirbelsäulenkrümmung, die durch den Cobb-Winkel repräsentiert wurde, besteht.

Im Abschnitt 4.5 wurde der Zusammenhang zwischen der Rotation von Wirbeln und Fehllagerate diskutiert. Eine zunehmende Rotation der Wirbel zur Vertikalen erhöht das Risiko einer Fehllage. Dies steht im Kontrast zu den Zusammenhängen zwischen CobbWinkel und Fehllage, bei dem keine Signifikanz nachgewiesen werden konnte.

Es ist also durchaus möglich, dass nur die Rotation, nicht jedoch die Skoliose der Wirbelsäule, einen Risikofaktor für Fehllagen darstellt. Dennoch sollte hierbei erwähnt werden, dass aufgrund der geringen Fallzahl die Aussagekraft der Signifikanz für den Cobb-Winkel abgeschwächt und damit mit Vorsicht anzunehmen ist. Insbesondere gilt dies, da es Hinweise darauf gibt, dass bei Skoliosepatienten mit starker Deformität der Eintrittswinkel in den Pedikel durch den SpineAssist ${ }^{\circledR}$ nicht immer erreicht werden kann (Barzilay et al. 2006; Hu et al. 2013). Um dies $\mathrm{zu}$ bestätigen, ist eine Untersuchung mit einer deutlich größeren Fallzahl an Patienten mit Skoliose notwendig. 


\subsection{Anzahl fusionierter Wirbel}

Die aktuelle Datenlage hinsichtlich der Zusammenhänge der Positionierung von Pedikelschrauben und der Anzahl fusionierten Wirbel ist mangelhaft. In der Metaanalyse von Verma et al. (2010) wurden 23 Studien auf das funktionelle Outcome von navigationsassitierter Pedikelverschraubung und ihre Fehllageraten untersucht. Auch weitere Studien, die sich mit den Problematiken der Pedikelverschraubung befassen, enthalten keine Information über die Anzahl der fusionierten Wirbel bei Spondylodese. Yoshii et al. (2015) stellten in ihrer Studie bei konventioneller Operationstechnik fest, dass signifikante Risikofaktoren für Fehllagen Fettleibigkeit und degenerative Skoliose sind. Esses et al. (1993) befassten sich mit möglichen Komplikationen bei der Verschraubung von Pedikelschrauben.

Die vorliegende Untersuchung beschäftigte sich jedoch mit dem Zusammenhang zwischen der Anzahl fusionierter Wirbel und der Fehllagerate der roboterassistierten perkutanen Pedikelverschraubung. 127 Patienten erhielten vier Pedikelschrauben, die in zwei Wirbel gesetzt wurden, womit die Versteifung von einem Segment erreicht wurde. Bei diesen Patienten wiesen 31 Fehllagen auf, insgesamt lagen 35 von 124 Schrauben fehl $(28,2 \%)$.

Drei Patienten erhielten eine Anschluss-Spondylodese, bei der zwei Schrauben in einen Wirbel eingesetzt wurden. Hierbei traten keine Fehllagen auf.

Dieses Risiko einer Schraubenfehllage stieg um $18 \%$ pro zusätzlich verschraubten Wirbel. Damit wird deutlich, dass eine genaue Neujustierung des SpineAssist ${ }^{\circledR}$ vor Insertion bei jedem Wirbel wichtig ist, um das Risiko einer möglichen Fehllage zu reduzieren.

Die Pedikelschrauben werden nach Implantation über ein Stabsystem verbunden. Es ist davon auszugehen, dass die Pedikelschrauben nach Insertion fest im Knochen verankert sind und sich trotz der Scherkräfte, die über den Stab auf die Schrauben ausgeübt werden, ihre Position nicht mehr verändert. Selbst bei Reinsertion in eine bereits verwendete Bohrbahn konnte eine ausreichende Stabilität der Pedikelschrauben im Knochen erreicht werden (Kang et al. 2014). Dennoch ist bekannt, dass Implantate in osteoporotischem und osteopenischem Knochen eine geringere Festigkeit im Knochen erreichen und im Verlauf häufiger Instabilitäten aufweisen können (Chen et al. 2011; Cook et al. 2001). Daher könnten durch eine zunehmende Anzahl fusionierter Wirbel auch die Scherkräfte zunehmen, die unter bestimmten Faktoren die Position der 
Pedikelschrauben nach bereits erfolgter Insertion verändern. Diese Problematik sollte in weiteren Studien untersucht werden.

\subsection{Komplikationen und Reoperation}

Insgesamt liegt die Komplikationsrate beim Einsatz des SpineAssist ${ }^{\circledR}$ in einem äußerst geringen Bereich. Lediglich 35 von 910 implantierten Schrauben (3,8\%) wiesen eine Abweichung von $>3 \mathrm{~mm}$ auf und wurden damit als Grad II klassifiziert. Eine Abweichung Grad III oder IV fehlte in diesem Patientenkollektiv gänzlich. Insgesamt hatten 23 Patienten mindestens eine Schraube mit einem Grad II, davon wiesen vier Patienten postoperativ neurologische Beschwerden auf. Eine Reoperation war bei drei Patienten indiziert, die Lumbalgien eines Patienten konnten erfolgreich konservativ therapiert werden.

Diese niedrigen Komplikationsraten decken sich mit den Befunden anderer Studien über den Einsatz des SpineAssist ${ }^{\circledR}$. Sie fanden in 0- 0,8 \% postoperativ neurologische Defizite vor (Devito et al. 2010; Hu et al. 2013). In einer Studie von Kantelhardt et al. (2011), in der die Ergebnisse roboterassistierter mit konventioneller Operationstechnik verglichen wurden, war in $1 \%$ der durch den SpineAssist ${ }^{\circledR}$ platzierten Schrauben eine Revision nötig. Anzumerken ist jedoch, dass nur 0,3\% dieser Fehlplatzierung in perkutaner Technik durchgeführt worden waren. Somit kann von einem risikoarmen und sicheren Einsatz des SpineAssist ${ }^{\circledR}$ bei Spondylodese ausgegangen werden.

Hinzuzufügen ist, dass im vorliegenden Patientenkollektiv dieser Arbeit neurologische Defizite innerhalb der von Gertzbein und Robbins (1990) definierten sicheren Zone auftraten. Dies verdeutlicht, dass neurologische Komplikationen auch bei geringer Abweichung der Schraube möglich sind, wodurch die Bedeutung einer genauen Analyse der Schraubenabweichung umso deutlicher wird 


\section{Zusammenfassung}

Die vorliegende Arbeit untersuchte die Genauigkeit des SpineAssist ${ }^{\circledR}$ und verschiedene Parameter, die eine mögliche Fehlerquelle für abweichende Pedikelschrauben bei roboterassistierter Spondylodese darstellen.

Die Ergebnisse der Genauigkeit der postoperativen Schraubenlage ist mit den in der Literatur angebebenen Werten konform, bei denen Fehllageraten zwischen 6- $8 \%$ nachgewiesen wurden (s. Abschnitt 4.1). Die Revisionsrate der roboterassistierten perkutan eingebrachten Schrauben lag bei Kantelhardt et al. (2011) bei 0,3\% und ist mit dem vorliegenden Ergebnis dieser Arbeit vergleichbar, bei dem die Revisionsrate aufgrund deplatzierter Schrauben $0 \%$ betrug. Die geringe Prozentzahl an Fehllagen überzeugt und macht den Einsatz des SpineAssist ${ }^{\circledR}$ bei perkutaner Spondylodese zulässig.

Die untersuchten Parameter, wie Alter, Geschlecht, Gewicht und Knochendichte, sind kein Ausschlusskriterium für eine roboterassistierte Spondylodese. Dies ist insofern vorteilhaft, als damit ein größeres Patientenspektrum trotz gewisser Dispositionen ebenfalls von der besseren Genauigkeit des SpineAssist ${ }^{\circledR}$ profitieren kann.

Die präoperative Analyse der Wirbelrotation im betroffenen Wirbelsäulenabschnitt ist für die Planung der Spondylodese unerlässlich. Fehllagen werden durch eine zunehmende Rotation und Differenz der Rotationsstärken sowie durch Spondylolisthesis innerhalb des OP-Gebiets begründet. Durch eine sorgsame Planung kann das Risiko im Vorhinein erkannt und entsprechend reduziert werden. Daher ist nach Auswertung der vorliegenden Datenerhebung eine genaue Analyse der Wirbelrotation und eine Neujustierung des Roboters bei langstreckiger Spondylodese zu empfehlen.

Um jedoch einen verbesserten internationalen Vergleich der Schraubenfehllagen durchführen zu können, sollte für zukünftige Studien die Einführung eines einheitlichen Klassifikationssystems der Pedikelschraubenfehllage angestrebt werden.

Roboterassistierte Wirbelsäulenchirurgie stellt eine Verbesserung der bisherigen Operationstechniken dar. Die Genauigkeit der roboterassistierten Wirblsäulenchirurgie ist im Vergleich zu anderen Operationstechniken bisher die mit der größten Effizienz. Der SpineAssist ${ }^{\circledR}$ ist in der Lage, präzise zu arbeiten. Menschliche Schwächen wie 
Erschöpfung und physiologischer Handtremor können ausgeschlossen werden. Er ist nicht nur für das Einsetzen von Pedikelschrauben von Bedeutung, sondern kann auch bei der Vertebroplastie, der Kyphoplastie und der Biopsie von Wirbelkörpertumoren, insbesondere im oberen und mittleren BWS-Bereich, zum Einsatz kommen, was ihn zusätzlich qualifiziert.

Dennoch sollte die Ursachenforschung für Fehllagen weiter vorangetrieben werden, um die Fehllageraten weiter zu reduzieren und möglichen Komplilkationen vorzubeugen. 


\section{Literaturverzeichnis}

Aaro S, Dahlborn M, Svensson L (1978): Estimation of vertebral rotation in structural scoliosis by computer tomography. Acta Radiol Diagn (Stockh) $\underline{19}$, 990-992

Amiot LP, Lang K, Putzier M, Zippel H, Labelle H (2000): Comparative results between conventional and computer-assisted pedicle screw installation in the thoracic, lumbar, and sacral spine. Spine $\underline{25}, 606-614$

Arand M, Hartwig E, Hebold D, Kinzl L, Gebhard F (2001): Präzisionsanalyse navigationsgestützt implantierter thorakaler und lumbaler Pedikelschrauben - Eine prospektive klinische Studie. Unfallchirurg 104, 1076-1081

Austin MS, Vaccaro AR, Brislin B, Nachwalter R, Hilibrand AS, Albert TJ (2002): Image-guided spine surgery: a cadaver study comparing conventional open laminoforaminotomy and two image-guided techniques for pedicle screw placement in posterolateral fusion and nonfusion models. Spine 27, 2503-2508

Barzilay Y, Liebergall M, Fridlander A, Knoller N (2006): Miniature robotic guidance for spine surgery - introduction of a novel system and analysis of challenges encountered during the clinical development phase at two spine centres. Int $\mathrm{J}$ Med Robot $\underline{2}, 146-153$

Bradford DS, Tay BKB, Hu SS (1999): Adult scoliosis: surgical indications, operative management, complications, and outcomes. Spine 24, 2617-2629

Celenk C, Celenk P: Bone density measurement using computed tomography. In: Saba L (Hrsg.): Computed Tomography - Clinical Applications. In Tech, o.O. 2012, 123-136

Chen LH, Tai CL, Lee DM, Lai PL, Lee YC, Niu CC, Chen WJ (2011): Pullout strength of pedicle screws with cement augmentation in severe osteoporosis: A comparative study between cannulated screws with cement injection and solid screws with cement pre-filling. BMC Musculoskelet Disord 12, 33

Cobb JR (1948): Outline for the study of scoliosis. Am Acad Orthop Surg Lect $\underline{5}$, 26175 
Coe JD, Warden KE, Herzig MA, McAFEE PC (1990): Influence of bone mineral density on the fixation of thoracolumbar implants - a comparative study of transpedicular screws, laminar hooks, and spinous process wires. Spine $\underline{15}$, 902-907

Cook SD, Barbera J, Rubi M, Salkeld SL, Whitecloud TS (2001): Lumbosacral fixation using expandable pedicle screws. An alternative in reoperation and osteoporosis. Spine $\mathrm{J} \underline{1}, 109-114$

Curtin F, Morabia A, Pichard C, Slosman DO (1997): Body mass index compared to dual-energy x-ray absorptiometry: Evidence for a spectrum bias. J Clin Epidemiol 50, $837-843$

Devito DP, Kaplan L, Dietl R, Pfeiffer M, Horne D, Silberstein B, Hardenbrook M, Kiriyanthan G, Barzilay Y, Bruskin A (2010): Clinical acceptance and accuracy assessment of spinal implants guided with SpineAssist surgical robot: retrospective study. Spine $\underline{35}, 2109-2115$

Dick W, Kluger P, Magerl F, Woersdörfer O, Zäch G (1985): A new device for internal fixation of thoracolumbar and lumbar spine fractures: the "fixateur interne". Paraplegia $\underline{23}, 225-232$

Dickman CA, Fessler RG, MacMillan M, Haid RW (1992): Transpedicular screw-rod fixation of the lumbar spine: operative technique and outcome in 104 cases. J Neurosurg $\underline{77}, 860-870$

Esses SI, Sachs BL, Dreyzin V (1993): Complications associated with the technique of pedicle screw fixation. A selected survey of ABS members. Spine 18, 2231-2239

Farber GL, Place HM, Mazur RA, Jones DEC, Damiano TR, (1995): Accuracy of pedicle screw placement in lumbar fusions by plain radiographs and computed tomography. Spine 20, 1494-1499

Ferguson AB (1930): The Study and Treatment of Scoliosis. South Med J 23, 116-120

Friberg O (1987): Lumbar Instability: A dynamic approach by traction-compression radiography. Spine $\underline{12}, 119-129$

Gallagher D, Visser M, Sepúlveda D, Pierson RN, Harris T, Heymsfield SB (1996): How useful is body mass index for comparison of body fatness across age, sex, and ethnic groups? Am J Epidemiol 143, 228-239 
Gertzbein SD, Robbins SE (1990): Accuracy of pedicular screw placement in vivo. Spine 15, 11-14

Gertzbein SD, Macmichael D, Tile M (1982): Harrington instrumentation as a method of fixation in fractures of the spine. J Bone Joint Surg Br $\underline{64}, 526-529$

Göçen S, Aksu MG, Baktiroğlu L, Özcan Ö (1998): Evaluation of computed tomographic methods to measure vertebral rotation in adolescent idiopathic scoliosis: an intraobserver and interobserver analysis. J Spinal Disord 11, 210-214

Grass R, Biewener A, Dickopf A, Rammelt S, Heineck J, Zwipp H (2006): Perkutane dorsale versus offene Instrumentation bei Frakturen des thorakolumbalen Übergangs eine vergleichende prospektive Untersuchung. Unfallchirurg 109, 297-305

Halm H, Niemeyer T, Link T, Liljenqvist U (2000): Segmental pedicle screw instrumentation in idiopathic thoracolumbar and lumbar scoliosis. Eur. Spine J. $\underline{9}, 191-$ 197

Haun DW, Kettner NW (2005): Spondylolysis and spondylolisthesis: a narrative review of etiology, diagnosis, and conservative management. J Chiropr Med 4, 206-217

Ho EKW, Upadhyay SS, Chan FL, Hsu LCS, Leong JCY (1993): New methods of measuring vertebral rotation from computed tomographic scans. An intraobserver and interobserver study on girls with scoliosis. Spine $\underline{18}, 1173-1177$

Holly LT, Foley KT (2003): Three-dimensional fluoroscopy-guided percutaneous thoracolumbar pedicle screw placement. Technical note. J Neurosurg 99, 324-329

Hou S, Hu R, Shi Y (1993): Pedicle morphology of the lower thoracic and lumbar spine in a chinese population. Spine $\underline{18}, 1850-1855$

Hu X, Lieberman IH (2014): What is the learning curve for robotic-assisted pedicle screw placement in spine surgery? Clin Orthop Relat Res $\underline{472}$, 1839-1844

Hu X, Ohnmeiss DD, Lieberman IH (2013): Robotic-assisted pedicle screw placement: lessons learned from the first 102 patients. Eur Spine J 22, 661-666

Jerosch J, Malms J, Castro WH, Wagner R, Wiesner L (1992): Lagekontrolle von Pedikelschrauben von instrumentierter dorsaler Fusion der Lendenwirbelsäule. Z Orthop $\underline{130}, 479-483$ 
Jin M, Liu Z, Liu X, Yan H, Han X, Qiu Y, Zhu Z (2015): Does intraoperative navigation improve the accuracy of pedicle screw placement in the apical region of dystrophic scoliosis secondary to neurofibromatosis type I: comparison between O-arm navigation and free-hand technique. Eur Spine J 25, 1729-1737

Kang DG, Lehman RA, Wagner SC, Bevevino AJ, Bernstock JD, Gaume RE, Dmitriev AE (2014): Pedicle screw reinsertion using previous pilot hole and trajectory does not reduce fixation strength. Spine $\underline{39}, 1640-1647$

Kantelhardt SR, Martinez R, Baerwinkel S, Burger R, Giese A, Rohde V (2011): Perioperative course and accuracy of screw positioning in conventional, open roboticguided and percutaneous robotic-guided, pedicle screw placement. Eur Spine J 20, 860868

Kim YJ, Lenke LG, Cheh G, Riew KD (2005): Evaluation of pedicle screw placement in the deformed spine using intraoperative plain radiographs: a comparison with computerized tomography. Spine $\underline{30}, 2084-2088$

Kim MC, Chung HT, Cho JL, Kim DJ, Chung NS (2011): Factors affecting the accurate placement of percutaneous pedicle screws during minimally invasive transforaminal lumbar interbody fusion. Eur Spine J $\underline{20}, 1635-1643$

Kim HJ, Lee SH, Chang BS, Lee CK, Lim TO, Hoo LP, Yi JM, Yeom JS (2015): Monitoring the quality of robot-assisted pedicle screw fixation in the lumbar spine by using a cumulative summation test. Spine $\underline{40}, 87-94$

King D (1944): Internal fixation for lumbosacral fusion. Am J Surg $\underline{66}, 357-361$

Kosmopoulos V, Schizas C (2007): Pedicle screw placement accuracy: a meta-analysis. Spine $\underline{32}$, E111-120

Kotani Y, Abumi K, Ito M, Takahata M, Sudo H, Ohshima S, Minami A (2007): Accuracy analysis of pedicle screw placement in posterior scoliosis surgery: comparison between conventional fluoroscopic and computer-assisted technique. Spine $\underline{32}, 1543-1550$

Krag MH, Weaver DL, Beynnon BD, Haugh LD (1988): Morphometry of the thoracic and lumbar spine related to transpedicular screw placement for surgical spinal fixation. Spine $\underline{13,27-32}$ 
La Rosa, G, Conti A, Cacciola F, Cardali S, La Torre D, Gambadauro NM, Tomasello F (2003): Pedicle screw fixation for isthmic spondylolisthesis: does posterior lumbar interbody fusion improve outcome over posterolateral fusion? J Neurosurg $\underline{99}, 143-150$

Laine T, Makitalo K, Schlenzka D, Tallroth K, Poussa M, Alho A (1997 a): Accuracy of pedicle screw insertion: A prospective CT study in 30 low back patients. Eur Spine $\mathrm{J}$ $\underline{6}, 402-405$

Laine T, Schlenzka D, Mäkitalo K, Tallroth K, Nolte LP. Visarius H (1997 b): Improved accuracy of pedicle screw insertion with computer-assisted surgery. A prospective clinical trial of 30 patients. Spine 22, 1254-1258

Laine T, Lund T, Ylikoski M, Lohikoski J, Schlenzka D (2000): Accuracy of pedicle screw insertion with and without computer assistance: a randomised controlled clinical study in 100 consecutive patients. Eur Spine J $\underline{9}, 235-240$

Lehmann W, Ushmaev A, Ruecker A, Nuechtern J, Grossterlinden L, Begemann PG, Baeumer T, Rueger JM, Briem D (2008): Comparison of open versus percutaneous pedicle screw insertion in a sheep model. Eur Spine J $\underline{17}$, 857-863

Lieberman IH, Togawa D, Kayanja MM, Reinhardt MK, Friedlander A, Knoller N, Benzel EC (2006): Bone-mounted miniature robotic guidance for pedicle screw and translaminar facet screw placement: Part I - Technical development and a test case result. Neurosurgery $\underline{59}$, 641-650

Lieberman IH, Hardenbrook MA, Wang JC, Guyer RD (2012): Assessment of pedicle screw placement accuracy, procedure time, and radiation exposure using a miniature robotic guidance system. J Spinal Disord Tech. 25, 241-248

Liljenqvist UR, Halm HFH, Link TM (1997): Pedicle screw instrumentation of the thoracic spine in idiopathic scoliosis. Spine 22, 2239-2245

Lim MR, Girardi FP, Yoon SC, Huang RC, Cammisa FPJ (2005): Accuracy of Computerized Frameless Stereotactic Image-Guided Pedicle Screw Placement into Previously Fused Lumbar Spines. Spine 30, 1793-1798

Linhardt O, Götz J, Renkawitz T, Forster T, Kröber M, Grifka J: Erkrankungen und Verletzungen der Wirbelsäule. In Grifka J, Kuster M (Hrsg.): Orthopädie und Unfallchirurgie. Springer Verlag, Berlin 2011, 401-476 
Lonstein JE, Denis F, Perra JH, Pinto MR, Smith MD, Winter RB (1999):

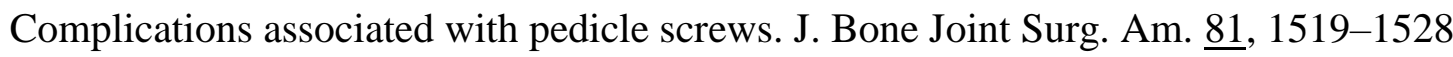

Magerl F (1980): Verletzungen der Brust- und Lendenwirbelsäule. Langenbecks Arch Chir $\underline{352}, 427-433$

Matsuzaki H, Tokuhashi Y, Matsumoto F, Hoshino M, Kiuchi T, Toriyama S (1990): Problems and solutions of pedicle screw plate fixation of lumbar spine. Spine $\underline{15}, 1159$ 1165

Matthews PG, Phan K, Rao PJ, Ball JR (2015): Pedicle length and degree of slip in lumbosacral isthmic spondylolisthesis. Orthop Surg 7, 108-111

Mazess R, Chesnut CH III, McClung M, Genant H (1992): Enhanced precision with dual-energy X-ray absorptiometry. Calcif Tissue Int $\underline{51}, 14-17$

McAfee PC, Weiland DJ, Carlow JJ (1991): Survivorship analysis of pedicle spinal instrumentation. Spine $\underline{16}, 422-427$

McCormack BM, Benzel E, Adams M, Baldwin N, Rupp FW, Maher DJ (1995): Anatomy of the thoracic pedicle. Neurosurgery 37, 303-308

Merloz P, Tonetti J, Pittet L, Coulomb M, Lavalleé S, Sautot P (1998): Pedicle screw placement using image guided techniques. Clin Orthop $\underline{354}, 39-48$

Meyerding HW (1932): Spondylolisthesis, Surg Gynecol Obstet 54, 371-377

Meyerding HW (1956): Spondylolisthesis; surgical fusion of lumbosacral portion of spinal column and interarticular facets; use of autogenous bone grafts for relief of disabling backache. J Int Coll Surg 26, 566-591

Misenhimer GR, Peek RD, Wiltse LL, Rothman SLG, Widell EH (1989): Anatomic analysis of pedicle cortical and cancellous diameter as related to screw size. Spine 14 , $367-372$

Morales-Avalos R, Leyva-Villegas J, Sánchez-Mejorada G, Cárdenas-Serna M, Vílchez-Cavazos F, Martínez-Ponce De León A, Elizondo-Riojas G, Martínez-García J, De La Garza-Castro O, Elizondo-Omaña R (2014): Age- and gender-related variations in morphometric characteristics of thoracic spine pedicle. Clin Anat 27, 441-450 
Mughir AM, Yusof MI, Abdullah S, Ahmad S (2010): Morphological comparison between adolescent and adult lumbar pedicle using computerized tomography scanning. Surg Radiol Anat $\underline{32}, 587-592$

Nash CL, Moe JH (1969): A Study of Vertebral Rotation. J Bone Joint Surg Am 51, 223-229

Niggemann P, Kuchta J, Beyer HK, Grosskurth D, Schulze T, Delank KS (2011): Spondylolysis and Spondylolisthesis, Prevalence of different forms of instability and clinical implications. Spine $\underline{36}$, E1463-E1468

Nilsson M, Johnell O, Jonsson K, Redlund-Johnell I (1988): Quantitative computed tomography in measurement of vertebral trabecular bone mass. A modified method Acta Radiol 29, 719-725

Nolte LP, Zamorano L, Visarius H, Berlemann U, Langlotz F, Arm E, Schwarzenbach O (1995 a): Clinical evaluation of a system for precision enhancement in spine surgery. Clin Biomech (Bristol Avon) 10, 293-303

Nolte LP, Zamorano LJ, Jiang Z, Wang Q, Langlotz F, Berlemann U (1995 b): Imageguided insertion of transpedicular screws. A laboratory set-up. Spine 20, 497-500

Norton MR, Gamble C (2001): Bone classification: an objective scale of bone density using the computerized tomography scan. Clin Oral Implants Res $\underline{12}, 79-84$

Olsewski JM, Simmons EH, Kallen FC, Mendel FC, Severin CM, Berens DL (1990): Morphometry of the lumbar spine: anatomical perspectives related to transpedicular fixation.J. Bone Joint Surg. Am., 72, 541 -549 .

Pandey MP, Schöggl K, Viszelj J, Widhalm K (2011): Body Composition: DualRöntgen-Absorptiometrie (DEXA). Journal für Ernährungsmedizin 13, 32-34

Pechlivanis I, Kiriyanthan G, Engelhardt M, Scholz M, Lücke S, Harders A, Schmieder, K (2009): Percutaneous placement of pedicle screws in the lumbar spine using a bone mounted miniature robotic system: first experiences and accuracy of screw placement. Spine $\underline{34}, 392-398$

Rajasekaran S, Vidyadhara S, Ramesh P, Shetty A.P (2007): Randomized clinical study to compare the accuracy of navigated and non-navigated thoracic pedicle screws in deformity correction surgeries. Spine $\underline{32}$, E56-64 
Regev GJ, Lee YP, Taylor WR, Garfin SR, Kim CW (2009): Nerve Injury to the Posterior Rami Medial Branch During the Insertion of Pedicle Screws: Comparison of Mini-Open Versus Percutaneous Pedicle Screw Insertion Techniques. Spine 34, 12391242

Rivet DJ, Jeck D, Brennan J, Epstein A, Lauryssen C (2004): Clinical outcomes and complications associated with pedicle screw fixation - augmented lumbar interbody fusion. J Neurosurg Spine 1, 261-266

Roser F, Tatagiba M, Maier G (2013): Spinal robotics: current applications and future perspectives. Neurosurgery $\underline{72}$, A12-A18

Roy-Camille R, Saillant G, Mazel C (1986): Internal fixation of the lumbar spine with pedicle screw plating. Clin Orthop 203, 7-17

Russell GG, Raso VJ, Hill D, McIvor J (1990): A comparison of four computerized methods for measuring vertebral rotation. Spine 15, 24-27

Rüther W, Lohmann CH: Orthopädie und Unfallchirurgie. 20. Auflage; Urban \& Fischer Verlag/Elsevier GmbH, München 2014

Schatlo B, Martinez R, Alaid A, von Eckardstein K, Akhavan-Sigari R, Hahn A, Stockhammer F, Rohde V (2015): Unskilled unawareness and the learning curve in robotic spine surgery. Acta Neurochir $\underline{157}, 1819-1823$

Schizas C, Michel J, Kosmopoulos V, Theumann N (2007): Computer tomography assessment of pedicle screw insertion in percutaneous posterior transpedicular stabilization. Eur Spine J 16, 613-617

Schnake KJ: CT-basierte Computernavigation von Pedikelschrauben an der Brustwirbelsäule - Praktikabilität, klinische Ergebnisse und Vergleich zur konventionellen Technik. Med Diss Berlin 2002 (http://edoc.huberlin.de/dissertationen/schnake-klaus-john-2002-07-12/PDF/Schnake.pdf. Zuletzt zugegriffen am 07.07.2015)

Schreiber JJ, Anderson PA, Rosas HG, Buchholz AL, Au AG (2011): Hounsfield units for assessing bone mineral density and strength: a tool for osteoporosis management. $\mathrm{J}$ Bone Joint Surg Am 93, 1057-1063 
Seller K, Wild A, Urselmann L, Krauspe R (2005): Prospektive Schraubenfehllagenanalyse nach konventioneller und navigierter Pedikelschraubenimplantation / Computer Assisted Orthopedic Surgery (CAOS). Biomed Tech Eng 50, 287-292

Shah NR, Braverman ER (2012): Measuring Adiposity in Patients: The Utility of Body Mass Index (BMI), Percent Body Fat, and Leptin. PloS One ㄱ, e33308

Shapurian T, Damoulis PD, Reiser GM, Griffin TJ, Rand WM (2006): Quantitative evaluation of bone density using the Hounsfield index. Int $\mathrm{J}$ Oral Maxillofac Implants $\underline{21}, 290-297$

Shoham M, Burman M, Zehavi E, Joskowicz L, Batkilin E, Kunicher Y (2003): Bonemounted miniature robot for surgical procedures: Concept and clinical applications. IEEE Trans Robot Autom 19, 893-901

Shoham M, Brink-Danan S, Friedlander A, Knoller N (2006): Bone-Mounted Miniature Robotic System for Spine Surgery, The First IEEE/RAS-EMBS International Conference on Biomedical Robotics and Biomechatronics, Pisa, 20-22.02.2006. BioRob 2006, 917-920

Sihvonen T, Herno A, Paljärvi L, Airaksinen O, Partanen J, Tapaninaho A (1993): Local denervation atrophy of paraspinal muscles in postoperative failed back syndrome. Spine $\underline{18}, 575-581$

Stenholm S, Harris TB, Rantanen T, Visser M, Kritchevsky SB, Ferrucci L (2008): Sarcopenic obesity: definition, cause and consequences. Curr Opin Clin Nutr Metab Care $\underline{11}, 693-700$

Stokes IAF, Bigalow LC, Moreland MS (1986): Measurement of axial rotation of vertebrae in scoliosis. Spine $\underline{11}, 213-218$

Suk SI, Lee CK, Min HJ, Cho KH, Oh JH (1994): Comparison of Cotrel-Dubousset pedicle screws and hooks in the treatment of idiopathic scoliosis. Int Ortop $\underline{18}, 341-346$

Sukovich W, Brink-Danan S, Hardenbrook M (2006): Miniature robotic guidance for pedicle screw placement in posterior spinal fusion: early clinical experience with the SpineAssist. Int J Med Robot 2, 114-122

Svendsen OL, Haarbo J, Hassager C, Christiansen C (1993): Accuracy of measurements of body composition by dual-energy x-ray absorptiometry in vivo. Am J Clin Nutr $\underline{57}$, 605-608 
Tian NF, Xu HZ (2009): Image-guided pedicle screw insertion accuracy: a metaanalysis. Int Orthop $\underline{33}, 895-903$

Togawa D, Kayanja MM, Reinhardt MK, Shoham M, Balter A, Friedlander A, Knoller N, Benzel EC, Lieberman IH (2007): Bone-mounted miniature robotic guidance for pedicle screw and translaminar facet screw placement: part 2 - Evaluation of system accuracy. Neurosurgery $\underline{60}$, ONS129-139

Uppot RN, Sahani DV, Hahn PF, Kalra MK, Saini SS, Mueller PR (2006): Effect of Obesity on Image Quality: Fifteen-year Longitudinal Study for Evaluation of Dictated Radiology Reports. Radiology 240, 435-439

Uppot RN, Sahani DV, Hahn PF, Gervais D, Mueller PR (2007): Impact of Obesity on Medical Imaging and Image-Guided Intervention. AJR Am J Roentgenol 188, 433-440

Vaccaro AR, Rizzolo SJ, Balderston RA, Allardyce TJ, Garfin SR, Dolinskas C, An HS (1995): Placement of pedicle screws in the thoracic spine. Part II: An anatomical and radiographic assessment. J Bone Joint Surg Am $\underline{77}$, 1200-1206

Van Dijk JD, van den Ende RPJ, Stramigioli S, Köchling M, Höss N (2015): Clinical pedicle screw accuracy and deviation from planning in robot-guided spine surgery: robot-guided pedicle screw accuracy. Spine 40, E986-91

Verma R, Krishan S, Haendlmayer K, Mohsen A (2010): Functional outcome of computer-assisted spinal pedicle screw placement: a systematic review and metaanalysis of 23 studies including 5,992 pedicle screws. Eur Spine J $\underline{19}$, 370-375

Weinstein JN, Spratt KF, Spengler D, Brick C, Reid S (1988): Spinal pedicle fixation: reliability and validity of roentgenogram-based assessment and surgical factors on successful screw placement. Spine $\underline{13}, 1012-1018$

Weinstein JN, Rydevik BL, Rauschning W (1992): Anatomic and technical considerations of pedicle screw fixation. Clin Orthop $\underline{284}$ 34-46

West JL III, Ogilvie JW, Bradford DS (1991): Complications of the variable screw plate pedicle screw fixation. Spine $\underline{16}, 576-579$

Whitecloud TS III, Butler JC, Cohen JL, Candelora PD (1989): Complications with the variable spinal plating system. Spine $\underline{14}, 472-476$ 
Wiesner L, Kothe R, Rüther W (1999): Anatomic evaluation of two different techniques for the percutaneous insertion of pedicle screws in the lumbar spine. Spine 24, 15991603

Wiesner L, Kothe R, Schulitz KP, Rüther W (2000). Clinical evaluation and computed tomography scan analysis of screw tracts after percutaneous insertion of pedicle screws in the lumbar spine. Spine $\underline{25}, 615-621$

Wimmer C, Gluch H, Nogler M, Walochnik N (2001): Treatment of idiopathic scoliosis with CD-instrumentation. Acta Orthop Scand $\underline{6}, 615-620$

Wu CH, Kao YH, Yang SC, Fu, TS, Lai PL, Chen WJ (2008): Supplementary pedicle screw fixation in spinal fusion for degenerative spondylolisthesis in patients aged 65 and over: Outcome after a minimum of 2 years follow-up in 82 patients. Acta Orthop $\underline{79}, 67-73$

Yoshii T, Hirai T, Yamada T, Sumiya S, Mastumoto R, Kato T, Enomoto M, Inose H, Kawabata S, Shinomiya K, Okawa A (2015): Lumbosacral pedicle screw placement using a fluoroscopic pedicle axis view and a cannulated tapping device. J Orthop Surg Research $\underline{10}, 79$

Zindrick MR, Wiltse LL, Doornik A, Widell EH, Knight GW, Patwardhan AG, Thomas JC, Rothman SL, Fields BT (1987): Analysis of the morphometric characteristics of the thoracic and lumbar pedicles. Spine 12, 160-166 


\section{Danksagung}

Eine wissenschaftliche Arbeit ist nie das Werk einer einzelnen Person, deshalb möchte ich mich bei den Menschen bedanken, die mir die Erstellung meiner Dissertation ermöglicht haben.

Herrn Prof. Dr. med. Veit Rohde danke ich für die Möglichkeit, dass ich mich mit diesem interessanten und aktuellen Thema auseinander setzen durfte, insbesondere in einer Zeit, in der Robotertechnologien in der Medizin neue Maßstäbe setzen.

Ein besonderer Dank gilt Herrn Prof. Dr. med. Ramón Martínez-Olivera für die Betreuung der Arbeit. Mit viel Fachwissen, konstruktiven Gesprächen und Ratschlägen stand er mir stets zur Seite.

Ebenso danke ich Herrn Dr. med. Philipp von Gottberg und Herrn Dr. med. Florian Roghmann, ohne die meine Dissertation in dieser Form nicht möglich gewesen wäre. 


\section{Lebenslauf}

Ich, Anina Isabel Hahn, wurde am 14.03.1989 in Bonn geboren. Meine Eltern, Marion Hahn-Blum und Christof Hahn, tauften mich römisch-katholisch. Meine Schulzeit absolvierte ich zunächst an der Schloßbachschule in Bonn-Röttgen, bis ich 1999 auf die erzbischöfliche Liebfrauenschule in Bonn ging, wo ich 2008 mein Abitur machte.

Von 2008 bis 2010 studierte ich an der Heinrich-Heine-Universität in Düsseldorf Humanmedizin. Nach dem erfolgreichen Abschluss des 1. Abschnitts der Ärztlichen Prüfung wechselte ich den Studienort und studierte von 2010 bis 2014 an der GeorgAugust-Universität in Göttingen. Mein Praktisches Jahr absolvierte ich an der GeorgAugust-Universität Göttingen, dem Universitätsspital Basel und dem Clemenshospital in Münster. Den 2. Abschnitt der Ärztlichen Prüfung beendete ich erfolgreich am 12. November 2014. Am 15. Juli 2015 habe ich meine Weiterbildung zur Internistin am Marienhospital in Düsseldorf begonnen. 